Bacteriological and Water-Quality Data Collected at Coastal Mississippi Sites Following Hurricane Katrina, September-October 2005

Data Series 174 


\section{Bacteriological and Water-Quality Data Collected at Coastal Mississippi Sites Following Hurricane Katrina, September-October 2005}

By Richard A. Rebich and Richard H. Coupe

Data Series 174

\section{U.S. Department of the Interior}

U.S. Geological Survey 


\section{U.S. Department of the Interior \\ Gale A. Norton, Secretary \\ U.S. Geological Survey \\ P. Patrick Leahy, Acting Director}

U.S. Geological Survey, Reston, Virginia 2005

For product and ordering information:

World Wide Web: http://www.usgs.gov/pubprod

Telephone: 1-888-ASK-USGS

For more information on the USGS - the Federal source for science about the Earth, its natural and living resources, natural hazards, and the environment:

World Wide Web: http://www.usgs.gov

Telephone: 1-888-ASK-USGS

Any use of trade, product, or firm names is for descriptive purposes only and does not imply Endorsement by the U.S. Government. Although this report is in the public domain, permission must be secured from the individual copyright owners to reproduce any copyrighted material contained within this report. 


\title{
Contents
}

\author{
Abstract \\ Introduction \\ Site selection \\ Bacteriological sampling sites \\ Water-quality sampling sites \\ Sample collection and analysis methods \\ Bacteriological samples \\ Water-quality samples \\ Quality-assurance procedures \\ Bacteriological sample processing and analysis \\ Results \\ Water-quality sample processing and analysis \\ Bacteriological results \\ Summary \\ Water-quality results \\ Acknowledgments \\ References
}

\section{Figures 1-5. Maps showing}

1. Bacteriological sampling sites in three coastal Mississippi counties

2. Bacteriological sampling sites in Hancock County, Mississippi

3. Bacteriological sampling sites in Harrison County, Mississippi

4. Bacteriological sampling sites in Jackson County, Mississippi

5. Water-quality sampling sites in southeastern Mississippi

\section{Tables}

1. Information for bacteriological sampling sites - Hancock, Harrison, and Jackson Counties, Mississippi - post Hurricane Katrina, September 19 through October 18, 2005

2. Information for surface water-quality sampling sites, southeastern Mississippi, post Hurricane Katrina, September 19-30, 2005.

3. Bottle requirements, filtering requirements, and sample-preservation techniques for water-quality samples collected in the aftermath of Hurricane Katrina, September through October 2005

4. Results of bacteriological analyses for water samples collected in the aftermath of Hurricane Katrina, coastal Mississippi Counties, September 19 - October 18, 2005

5. U.S. Environmental Protection Agency criteria for Enterococci and Escherichia coli in recreational waters

6. Results of bacteriological quality-assurance samples collected in the aftermath of Hurricane Katrina, coastal Mississippi Counties, September 19 - October 18, 2005

7. Constituents and compounds included for analysis for samples collected in the aftermath of Hurricane Katrina, southeastern Mississippi, September 19-30, 2005

8. Constituents and compounds detected in samples collected in the aftermath of Hurricane Katrina, southeastern Mississippi, September 19-30, 2005

9. Results of quality-assurance samples collected at water-quality sites in the aftermath of Hurricane Katrina, southeastern Mississippi, September 19-30, 2005 


\section{CONVERSION FACTORS, ABBREVIATIONS, AND ACRONYMS}

\begin{tabular}{lll}
\hline Multiply & By & To Obtain \\
\hline Length & \\
\hline foot (ft) & 0.3048 & meter (m) \\
\hline Volume & \\
\hline $\begin{array}{l}\text { gallon (gal) } \\
\text { gallon (gal) }\end{array}$ & 3.785 & liter (L) \\
\hline
\end{tabular}

Temperature in degrees Celsius $\left({ }^{\circ} \mathrm{C}\right)$ may be converted to degrees Fahrenheit $\left({ }^{\circ} \mathrm{F}\right)$ as follows:

${ }^{\circ} \mathrm{F}=\left(1.8 \mathrm{x}^{\circ} \mathrm{C}\right)+32$

Temperature in degrees Fahrenheit $\left({ }^{\circ} \mathrm{F}\right)$ may be converted to degrees Celsius $\left({ }^{\circ} \mathrm{C}\right)$ as follows:

${ }^{\circ} \mathrm{C}=\left({ }^{\circ} \mathrm{F}-32\right) / 1.8$

Horizontal coordinate information is referenced to the North American Datum of 1983 (NAD83).

Specific conductance is given in microsiemens per centimeter at 25 degrees Celsius

$\left(\mu \mathrm{S} / \mathrm{cm}\right.$ at $\left.25^{\circ} \mathrm{C}\right)$.

Concentrations of chemical constituents in water are given either in milligrams per liter $(\mathrm{mg} / \mathrm{L})$ or micrograms per liter $(\mu \mathrm{g} / \mathrm{L})$.

$\begin{aligned} \mathrm{ft} & \text { foot } \\ \mathrm{L} & \text { liter } \\ \mathrm{mL} & \text { milliliter } \\ \mathrm{mg} / \mathrm{l} & \text { milligrams per liter } \\ \mu \mathrm{g} / \mathrm{L} & \text { micrograms per liter } \\ \mu \mathrm{S} / \mathrm{cm} & \text { microsiemens per centimeter } \\ \mathrm{CFU} & \text { colony forming units } \\ \mathrm{CO} & \text { Colorado } \\ \mathrm{COD} & \text { chemical oxygen demand } \\ \text { E. coli } & \text { Escherichia coli } \\ \mathrm{MDEQ} & \text { Mississippi Department of Environmental Quality } \\ \mathrm{MPN} & \text { most probably number } \\ \mathrm{MS} & \text { Mississippi } \\ \text { NOAA } & \text { National Oceanic and Atmospheric Administration } \\ \text { NWQL } & \text { USGS National Water Quality Laboratory } \\ \text { PVC } & \text { polyvinyl chloride } \\ \text { RPD } & \text { relative percent difference } \\ \text { USEPA } & \text { U.S. Environmental Protection Agency } \\ \text { USGS } & \text { U.S. Geological Survey }\end{aligned}$




\section{ABSTRACT}

On August 29, 2005, Hurricane Katrina devastated coastal Mississippi with 150 mile-per-hour winds and a storm surge in excess of 20 feet. Katrina moved inland and wreaked destruction on a broad swath of eastern Mississippi. Some eastern Mississippi counties were left without power and water and some major roads were impassable for weeks. The possibility of disease transmission from contaminated water and contamination caused by chemical spills were major concerns.

As part of a multi-agency response to the disaster, the U.S. Geological Survey (USGS), in partnership with the Mississippi Department of Environmental Quality (MDEQ), established a network of sampling locations to determine the effects of the storm on surface-water quality along the Mississippi Gulf Coast. Water samples were collected weekly at 31 estuarine tributary sites and 13 beach monitoring sites in coastal Mississippi Counties - Hancock, Harrison, and Jackson - for a period of 5 weeks beginning September 19, 2005. Samples were collected by MDEQ and USGS, were transported to a temporary laboratory established at a USGS facility at Stennis Space Center near Bay St. Louis, MS, and were analyzed within 6 hours of collection. USGS analyzed the samples primarily for enterococci, which is the standard fecal indicator bacteria for brackish waters and can be used as a fecal indicator for freshwater. Enterococci densities were determined by the most probable value method. About 14 percent of the enterococci densities were less than the detection limit, and 81 percent were lower than U.S. Environmental Agency criteria for the protection of public health. Densities at several of the bacteriological sites increased during the second week of sampling possibly due to runoff associated with Hurricane Rita rainfall that occurred September 23-24, 2005. Quality-control data were reviewed to ensure that methods performed as expected.

USGS also collected 19 water-quality samples at 12 inland freshwater sites for a period of 2 weeks starting on September 19, 2005. Sampling sites were located near established USGS stream gages. Physical properties of the streams were measured on site. Waterquality samples were collected, processed, and preserved on site according to standard procedures and then shipped to the USGS National Water Quality Laboratory in Denver, CO, for analysis--except for biochemical oxygen demand samples, which were analyzed by the MDEQ laboratory in Pearl, MS. Each sample was analyzed for multiple constituents including nutrients, major ions, trace metals, modern-use and polar pesticides, wastewater compounds, volatile organic compounds, and degradate organic compounds. Most detections were below available State and Federal water-quality criteria for Mississippi streams. Overall, the results from the bacteriological and water-quality samples indicated no systematic contamination in the sampled streams in the aftermath of Hurricane Katrina.

\section{INTRODUCTION}

Hurricane Katrina made landfall in coastal Alabama, Mississippi, and Louisiana on August 29, 2005. The local population in the three coastal Mississippi Counties - Hancock, Harrison, and Jackson - was concerned about water quality in streams, bays, and estuaries of the Gulf Coast in the aftermath of the hurricane because of damage caused by flooding, wind, and storm surge. Nearly all of the municipal wastewater treatment facilities in the three coastal counties, as well as many north of the affected counties, were out of operation for an extended period of time due to widespread power outages. Many of the industrial operations in the region, such as oil refineries, chemical production plants, and timber processing plants, were also without power for an extended period of time. As a result, there were concerns about potential contamination of the bays and estuaries that are used for commercial, recreational, and subsistence fishing and shellfish harvesting along the Mississippi Gulf Coast. There were also concerns about the safety of workers involved in debris removal from these same estuarine locations.

The U.S. Geological Survey (USGS) partnered with the Mississippi Department of Environmental Quality (MDEQ) to collect bacteriological samples at tributary locations to the estuaries and bays and at selected MDEQ beach monitoring sites in the three Mississippi Gulf Coast counties. Bacteriological samples were collected weekly for 5 consecutive weeks beginning September 19, 2005, and were analyzed primarily for enterococci, which is the U.S. Environmental Protection Agency (USEPA) standard for fecalindicator bacteria for brackish waters (U.S. Environmental Protection Agency, 2000 and 2004a). Selected samples were also analyzed for Escherichia coli (E. Coli).

The USGS also collected water-quality samples at current real-time stream gage or crest-stage gage locations on inland freshwater streams tributary to the coastal estuaries and bays. Water-quality samples were collected weekly for 2 weeks beginning September 19 , 2005, and were analyzed for numerous constituents including nutrients, major ions, trace metals, modern-use and polar pesticides, wastewater compounds, volatile organic compounds, and degradate organic compounds.

This report documents the site-selection criteria, sample-collection and analysis methods, quality-assurance procedures, and analysis results of the bacteriological and water-quality samples collected along the Mississippi Gulf Coast in the aftermath of Hurricane Katrina. This information will be useful to agencies that continue to coordinate clean-up efforts, community leaders involved in industrial recovery, and natural resource managers leading environmental assessments and restoration. 


\section{SITE SELECTION}

Many Federal, State, and local agencies were involved in collecting environmental data in the aftermath of Hurricane Katrina. It was determined, through numerous conference calls to coordinate the field activities of the involved Federal agencies, that the USEPA and the National Oceanic and Atmospheric Administration (NOAA) would collect bacteriological and water-quality samples in the Mississippi Sound and in the primary bays and estuaries (St. Louis Bay, Biloxi Bay, Pascagoula Bay) along the Mississippi Gulf Coast (National Oceanic and Atmospheric Administration, 2005a; U.S. Environmental Protection Agency, 2005). In an effort to coordinate with the data-collection efforts of USEPA and NOAA and to avoid duplication, the USGS and MDEQ chose to sample locations along the beaches and at inland locations on small tributaries of the bays and estuaries. This section documents selection of the bacteriological and the water-quality sampling sites by the USGS and MDEQ.

\section{Bacteriological Sampling Sites}

USGS and MDEQ selected 31 near-coastal sites for bacteriological sampling (table 1). These sites were located near active or historical USGS streamflow gage, crest-stage gage (which measures the highest stage during a runoff event), or water-quality sampling sites on inland tributaries to bays and estuaries in the three Mississippi Gulf Coast counties. Figure 1 shows site locations in all three counties, and figures 2, 3, and 4 show site locations in each county - Hancock, Harrison, and Jackson - respectively. Although each of the 31 sites has a USGS station number and station name, they were also assigned a unique field number for quick reference. The field number begins with the initials of the county: HK, Hancock County Site, HN, Harrison County site, and JK, Jackson County site (table 1). For example, HK-1 is Hancock County site 1 (table 1).

The USGS and MDEQ also collected bacteriological samples at 13 "beach" sites (table 1; figs. 1, 2, 3, and 4). These sites were part of MDEQ's Mississippi Beach Monitoring Program, which began in 2000 (Mississippi Department of Environmental Quality, 2005). These sites were also assigned a unique field number similar to the nomenclature used for the near-coastal sites; for example, HKB-1 is Hancock County beach site 1 (table 1).

\section{Water-Quality Sampling Sites}

As Hurricane Katrina made landfall, its strength diminished slowly as it proceeded northward into Mississippi. The cities of Hattiesburg and Meridian, MS, experienced Category 2 and Category 1 force winds, respectively, throughout the day and evening after landfall. Widespread damage to trees resulting in power outages that lasted for days, and in some cases weeks, were common in southern and eastern Mississippi. Such damage could potentially be a source of contamination to rivers and streams. There were reports of numerous fish kills in the Pascagoula River Basin, and although depletion of dissolved oxygen caused by large amounts of debris flushing into the streams was the likely cause, increased toxicity due to possible chemical spills was also suspected. For these reasons, USGS and MDEQ agreed that inland freshwater sites, especially in the Pascagoula River Basin, were of concern and were, therefore, targeted for sampling.

Water-quality sampling sites included eight sites in the Pascagoula River Basin, and one site each in the Tchoutacabouffa, Biloxi, Wolf, and Jourdan River Basins (fig. 5). All of the water-quality sampling sites were located at active USGS real-time streamflow gages (satellite telemetry) except for the Tchoutacabouffa River sampling site, which is an active crest-stage gage. Table 2 lists the station number, station name, type of site, dates sampled, and latitude-longitude for each of the water-quality sampling sites.

\section{SAMPLE COLLECTION AND ANALYSIS METHODS}

The methods used to collect and process bacteriological and water-quality samples collected in this study are documented in this section. These methods were agreed upon by the USGS and MDEQ prior to sampling, and generally followed procedures outlined in the USGS National Field Manual for the Collection of Water-Quality Data (U.S. Geological Survey, variously dated; hereafter referred to in this report as USGS-NFM).

\section{Bacteriological Samples}

Each field crew collecting bacteriological samples was composed of one USGS and one MDEQ employee. During each site visit, the stream stage was measured relative to an established reference point, hydrologic conditions were noted, a water sample was collected, and the date and time of the sample were recorded. In general, the water sample was collected in a 300-milliliter (mL) polypropylene bottle attached to a weighted sampler, which was lowered over the side of the bridge near the center of flow in the stream. The polypropylene bottle was sterilized and sealed prior to use and contained a tablet of sodium thiosulfate, which minimizes the potential effects of residual anti-bacterial chemicals, such as chlorine, that could be present in the water at each sampling location. At the beach monitoring sites, a special sampler made of a large polyvinyl chloride (PVC) elbow joint attached to an 8-foot (ft) aluminum pole was used to collect the sample in the surf at the shoreline. This sampler was used so that field personnel could avoid wading into the surf to collect a sample in unsafe conditions caused by the large amount of debris along the beach and in the surf. All samplers were cleaned with either anti-bacterial spray or isopropyl alcohol and allowed to air-dry between sampling sites. Sample bottles were stored in a cooler filled with ice for transport to the lab and were analyzed within 6 hours of collection. 


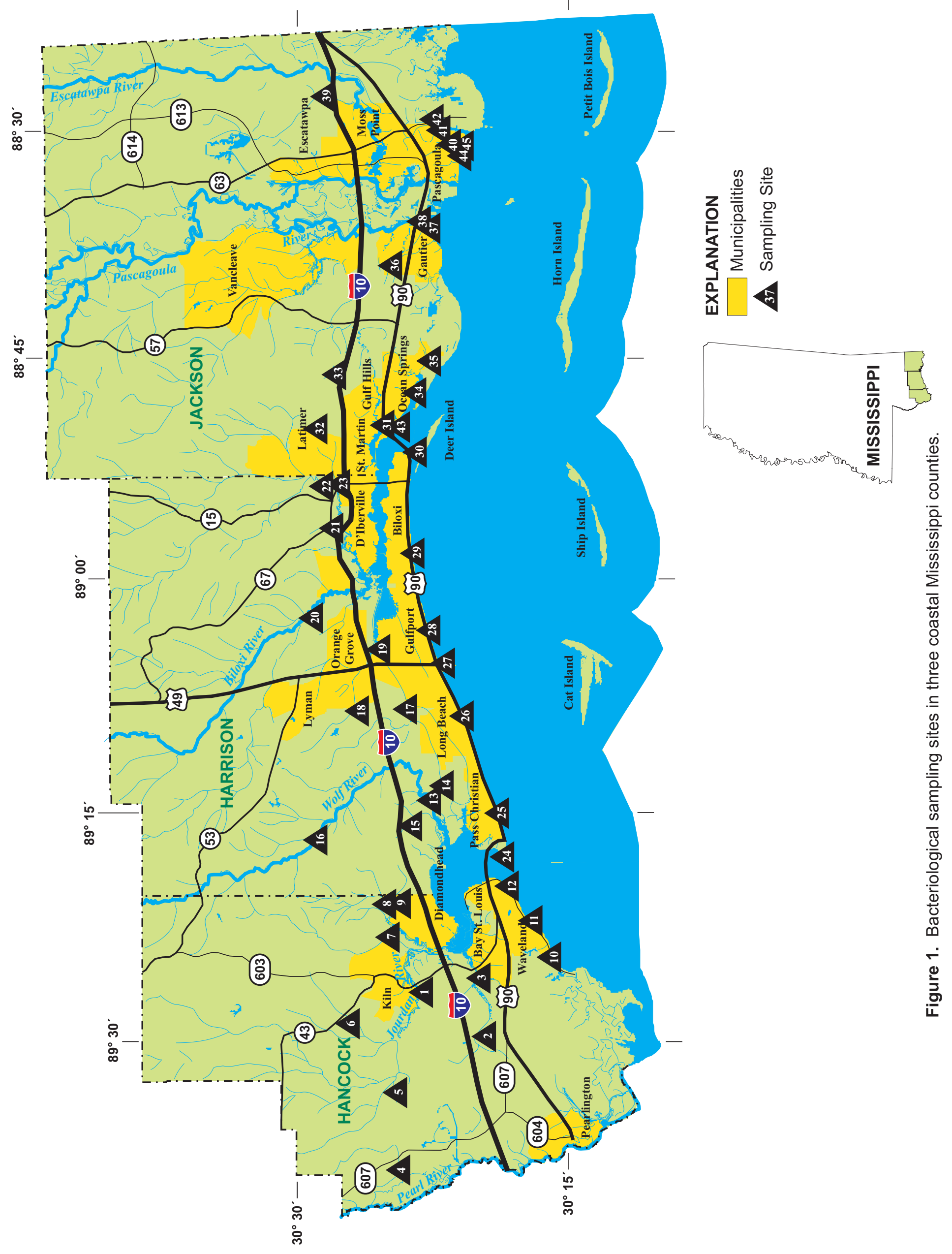




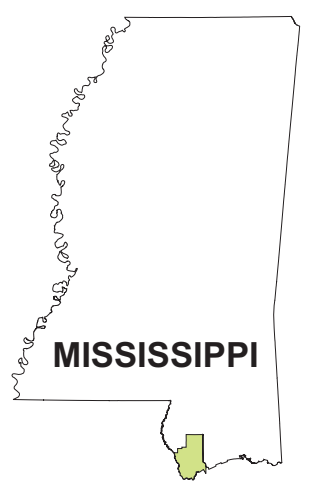

\section{EXPLANATION}

Municipalities

6 Sampling Site

$89^{\circ} 30^{\circ} \quad 89^{\circ} 22^{\prime} 30^{\prime \prime}$

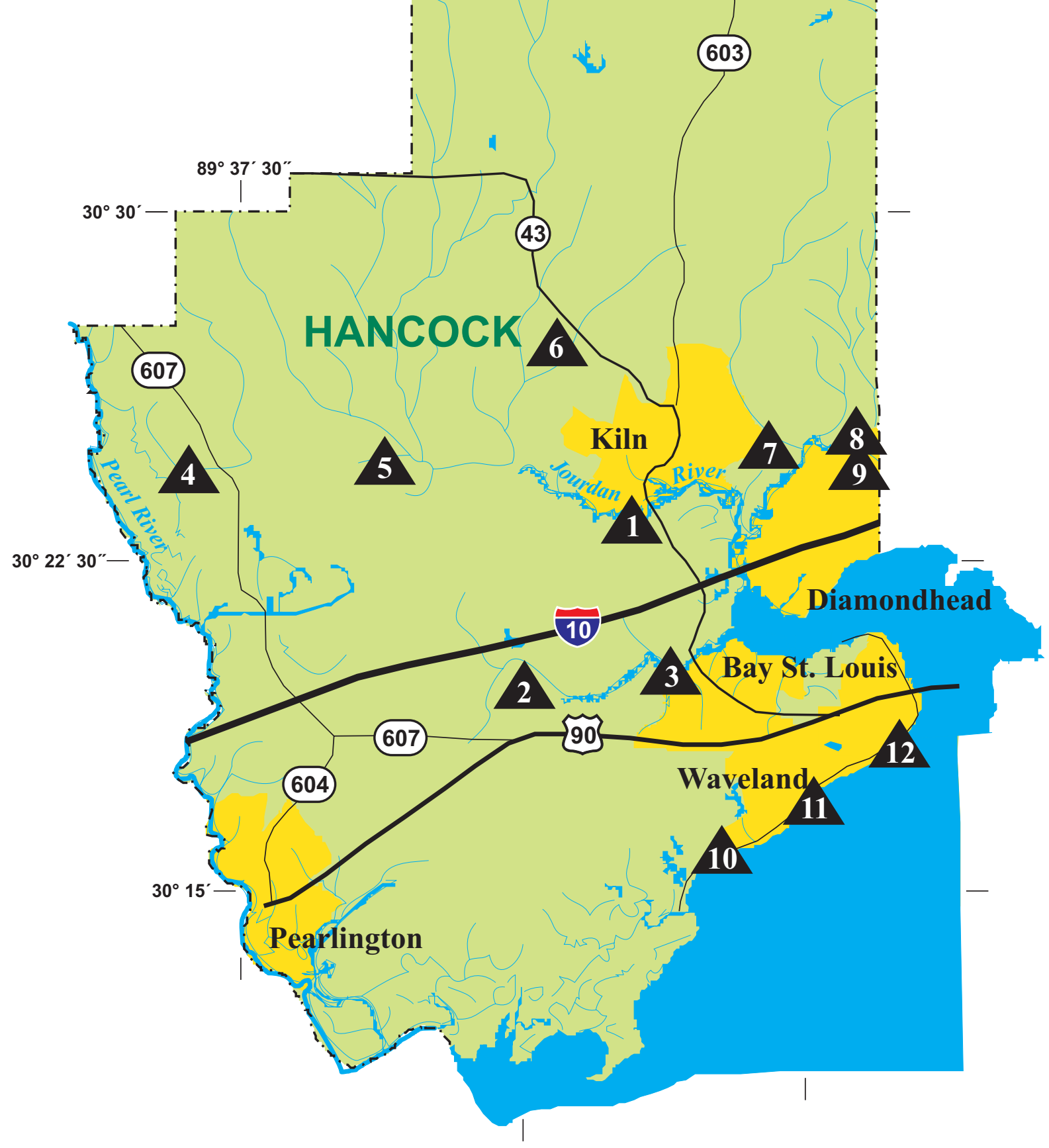

Figure 2. Bacteriological sampling sites in Hancock County, Mississippi. 


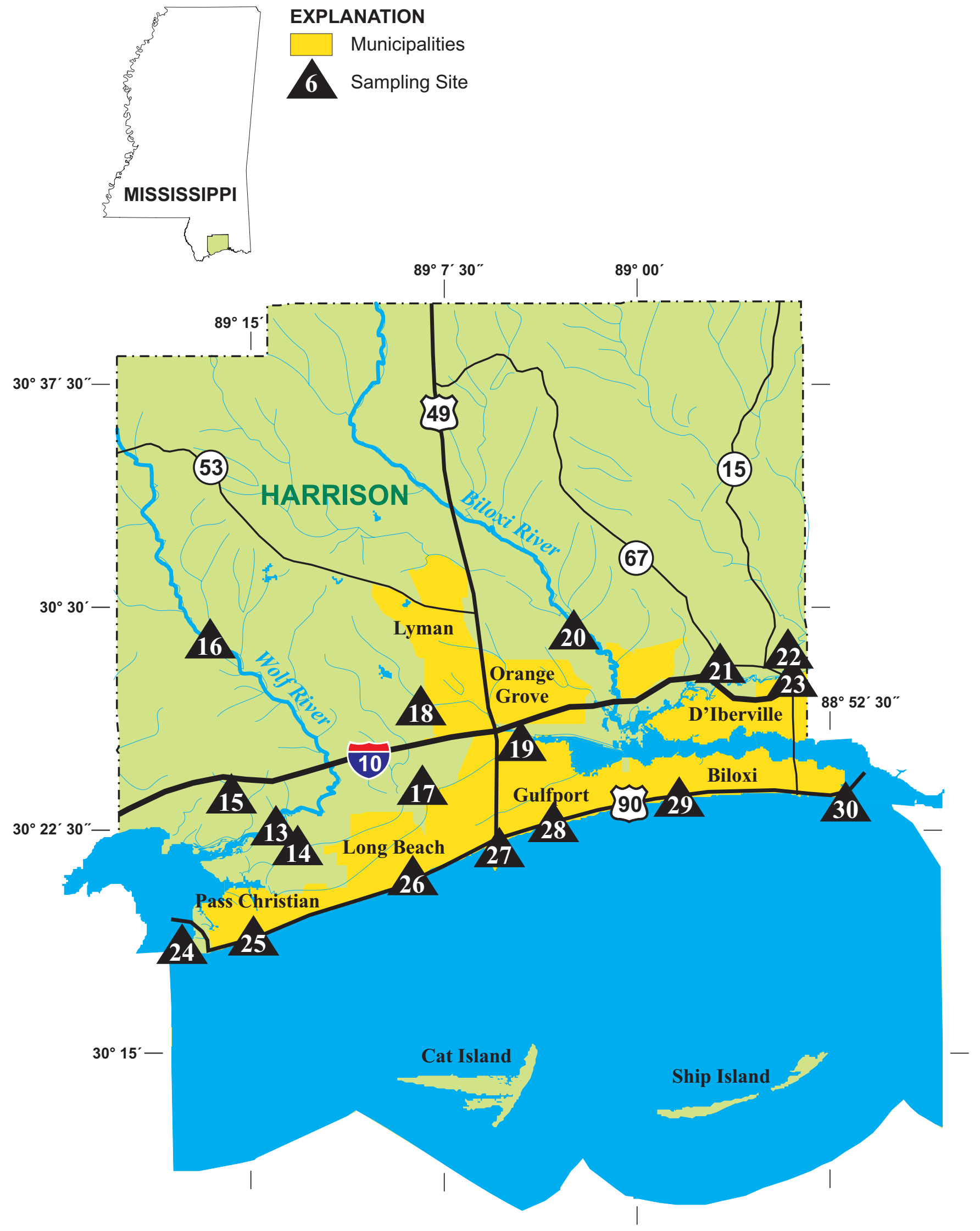

Figure 3. Bacteriological sampling sites in Harrison County, Mississippi. 

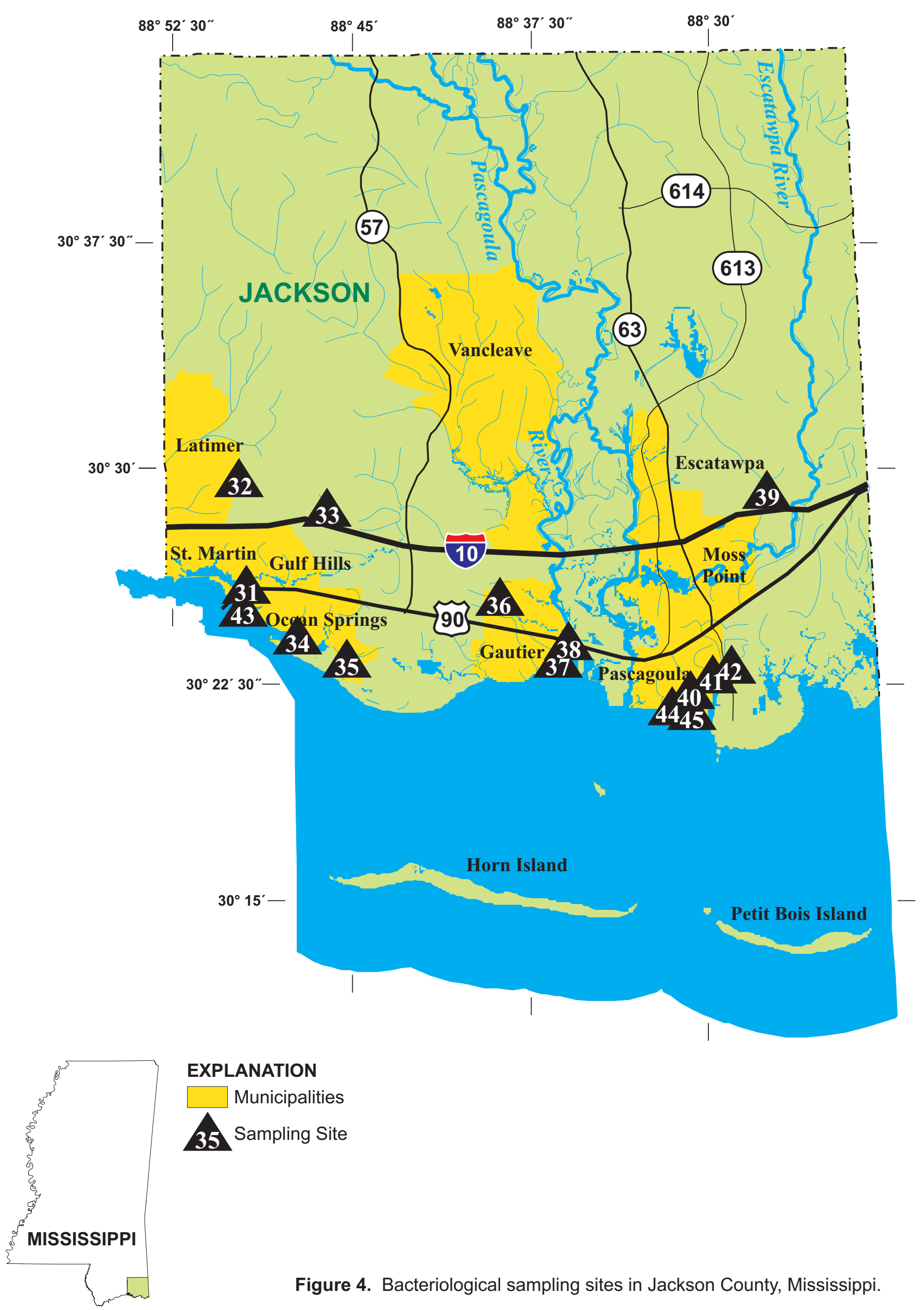

\section{EXPLANATION}

$\square$ Municipalities
35 Sampling Site

Figure 4. Bacteriological sampling sites in Jackson County, Mississippi. 


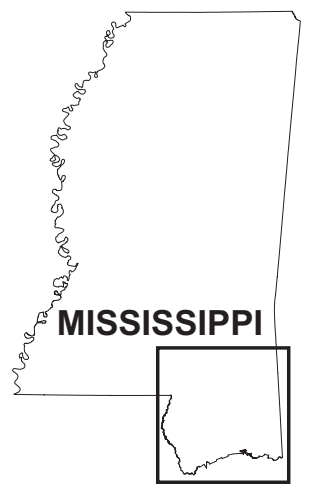

EXPLANATION

Municipalities

A Sampling Site

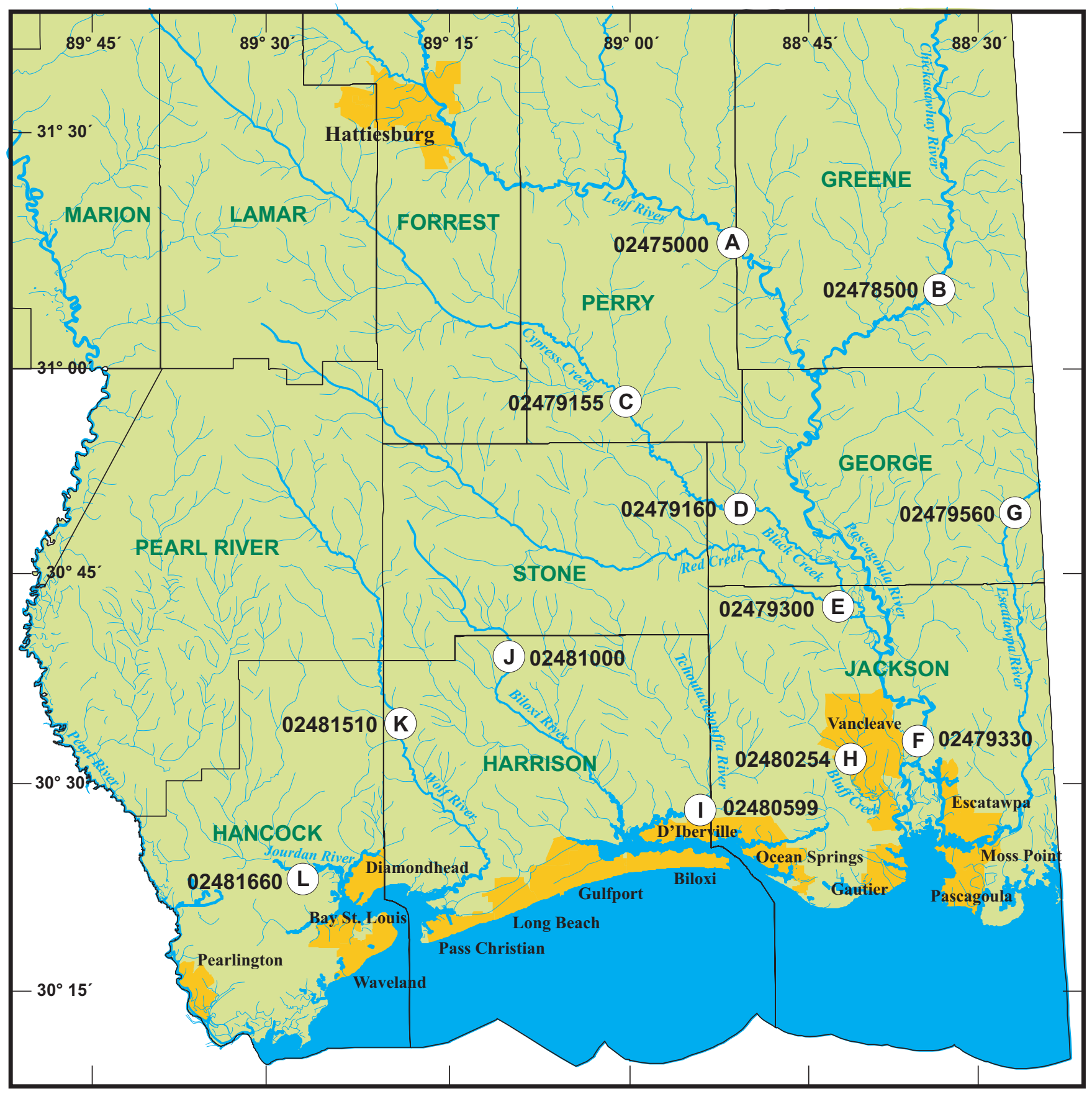

Figure 5. Water-quality sampling sites in southeastern Mississippi. 
Bacteriological samples were processed and analyzed by USGS employees at a temporary laboratory at the USGS Hydrologic Instrumentation Facility located on the property of the National Aeronautics and Space Administration's Stennis Space Center near Bay St. Louis, MS. When the samples were received at the laboratory, information about each sample was logged onto a lab analysis sheet, and a specific conductance reading was obtained from the sample bottle to determine if the sample water was considered freshwater or saltwater. All original field notes and lab sheets are available for inspection in the files of the USGS Mississippi Water Science Center.

Current USEPA recreational criteria for evaluating the microbiological quality of marine/brackish waters use an enterococci standard (U.S. Environmental Protection Agency, 2004a). Enterococci can also be used as an indicator of microbiological contamination in freshwater. Therefore, all bacteriological samples collected during this study were analyzed for enterococci.

There were two methods of analysis used to measure densities of enterococci in the samples. The primary method used for all samples was most-probable-number (MPN) using Enterolert defined-substrate medium (U.S. Environmental Protection Agency, 2003a) in Quanti-Tray 2000® wells (IDEXX Corporation, 2005a). MPN analyses result in a statistical estimate of the original density of cells in a water volume based on positive reactions (plus-minus response) in multiple sub-aliquots. Results of MPN tests are reported as MPN per $100 \mathrm{~mL}$. Confidence intervals were applied to MPN density estimates according to a tabulation of 95 percent confidence intervals provided by the manufacturer (IDEXX Corporation, 2005b).

Sample dilutions prior to analysis by MPN were based on whether the sample water was freshwater or saltwater. An undiluted, a 1:10 dilution, and in some cases, a 1:100 dilution were run on freshwater samples. A 1:10, and in some cases, a 1:100 dilution were run on saltwater samples. MPN analyses were not run on undiluted saltwater samples to avoid potential false positives caused by interference from a common marine bacillus bacteria as instructed by the manufacturer (Sharon Muhilly, IDEXX Corporation, oral commun., September 21, 2005).

About 15 percent of the samples were analyzed by membrane filtration methods for enterococci and for E. coli. Membrane filtration for enterococci was used as a means of comparison and verification of MPN results; E. coli analysis was used to further characterize the sampled waters for other fecal indicators. Enterococci and E. coli densities were determined by membrane filtration and cultivation on mEI agar and modified mTEC agar, respectively (U.S. Environmental Protection Agency, 2000). Membrane filtration results in a colony count from a defined volume of water, generally reported as colony forming units (CFU) per $100 \mathrm{~mL}$ water. Confidence intervals were not applied to bacteria densities measured by membrane filtration.

\section{Water-Quality Samples}

At each site, physical properties of the streams were measured near the center of flow at each site using a multi-probe which measured temperature, specific conductance, $\mathrm{pH}$, dissolved oxygen, and turbidity. Calibration of the multi-probe followed guidelines outlined in USGS-NFM (U.S. Geological Survey, variously dated). All instruments were calibrated each morning, and calibration was checked at the end of each day. Physical properties were recorded on field sheets, which are stored in files of the USGS Mississippi Water Science Center.

Prior to use, all equipment that came into contact with a water sample was cleaned with non-phosphate detergent, rinsed with deionized water, rinsed with methanol, air dried, and stored in a dust-free environment. All equipment (churn splitter, tubing, and bottles) was placed in plastic bags after cleaning to prevent contamination. Water samples were collected from bridges or by wading using established depth- and width-integrated procedures, and about 10 liters (L) of water were processed at each site using methods as outlined in the USGS-NFM (U.S. Geological Survey, variously dated). A Teflon churn splitter was used to subdivide each sample into appropriate bottles as required by the laboratory. Bottle requirements, filtering requirements, and sample-preservation techniques are listed in table 3. All samples were double-bagged in plastic bags, packed on ice, and shipped overnight to the USGS National Water Quality Laboratory (NWQL) in Denver, CO. The exception was for biochemical oxygen demand samples, which were collected in a 1-L polypropylene bottle, stored on ice and transported to the MDEQ laboratory in Pearl, MS, and were analyzed within 48 hours of collection. Between sites and at the end of each day of sampling, all equipment was cleaned and stored as specified earlier.

\section{QUALITY-ASSURANCE PROCEDURES}

This section documents the quality-assurance procedures for the processing and analysis of the bacteriological and water-quality samples. These procedures were followed to document potential bias and variability in the data sets.

\section{Bacteriological Sample Processing and Analysis}

Generally, quality-assurance practices for bacteriological analysis were followed as described by Francy and others (2005) and Myers and Wilde (2004). All sample collection and processing information was recorded on USGS microbiology field forms. Each day a media batch preparation form was completed to document the lot numbers of media and supplies used. The performance of the autoclaves was checked throughout the sampling period with heat-indicating autoclave tape to ensure that supplies were properly sterilized. Incubator temperatures were monitored with mercury thermometers at a minimum of twice daily, and results were recorded in a logbook. Each time the incubators were opened during the day, the temperature was checked and recorded on the field sheets. 
For the MPN Enterolert method, quality-control samples included duplicate samples, procedure and field blanks, and positive and negative controls. Generally, two water samples were analyzed weekly, in duplicate, to test analytical variability. Procedure blanks using deionized water were also analyzed at a frequency of two samples per week. A procedure blank is a blank solution that is processed through all stages of set-up and analysis to ensure that no contamination is introduced after samples were received at the lab. Only one field blank was processed for the study period. A field blank is a blank solution that is processed through all stages of sample collection to ensure that no contamination is introduced during the sample collection and handling phase prior to delivering the sample to the lab. Positive and negative control samples were grown on the MPN media to test the ability of the method to detect targeted bacteria and exclude non-targeted bacteria, respectively. The positive control bacteria were enterococci, and the negative control bacteria were E. coli. Positive and negative control samples were analyzed at a rate of once weekly.

For the membrane filtration method, quality-assurance samples included filter and procedure blanks, which were analyzed during sample processing to ensure that filtration equipment and buffer solutions were not contaminated. Filter and procedure blanks were analyzed at a rate of once every three to four samples processed.

\section{Water-Quality Sample Processing and Analysis}

Quality-assurance procedures generally followed those outlined in the USGS-NFM (U.S. Geological Survey, variously dated). As part of these procedures, quality-assurance samples collected during the study period included one sequential replicate, field blank, equipment blank, and laboratory spike. Sites were chosen at random for the collection and processing of these samples. A sequential replicate is an additional 10-L sample of environmental water collected immediately after an environmental sample is collected at a particular site. The sequential replicate is subjected to the same processing as the environmental sample and is used to assess variability among samples due to collection, processing, shipping, and analysis procedures for samples collected at different times. The sequential replicate is also used to assess water-quality variability in the stream being sampled.

For the field blank, laboratory-assured chemical-free water was processed at a field site in the same manner and with the same equipment used to process the environmental sample. A field blank is used to determine if there are any sources of contamination introduced by the sample collection and processing procedures that could bias results for the environmental samples. For the equipment blank, laboratory-assured chemical-free water was processed through the field equipment at the USGS office in Jackson, MS, prior to sampling to check initial cleaning procedures.

For the laboratory spike, an additional sample was collected at one site, processed in the same manner as the environmental sample, and shipped to the laboratory where a mix of known concentrations of target compound was added to the sample. The target compounds included organic chemicals such as pesticides, wastewater compounds, and volatile organic compounds. The laboratory spike samples were used to test the ability of the laboratory to accurately measure those particular compounds, to assess environmental matrix effects associated with the analysis, and to assess potential degradations in these compounds due to sample processing, shipping, or analysis.

\section{RESULTS}

Analytical results for the bacteriological and water-quality samples collected during this study period are stored in the USGS National Water Information System (NWIS) database. Results of the quality-assurance samples are stored locally in the USGS Mississippi Water Science Center NWIS quality-assurance database.

\section{Bacteriological Results}

Bacteriological data for MPN analyses of enterococci (including 95 percent confidence intervals) and membrane filtration of enterococci and E. coli are presented in table 4. The MPN test results are a statistical estimate of the density of enterococci in a water sample based on a presence/absence test in multiple sub-aliquots. Membrane filtration test results are based on an actual count of colonies forming on a nutrient agar medium under the conditions of the analysis. There were 200 samples analyzed for enterococci using the Enterolert MPN method and 30 samples analyzed for enterococci and E. coli using the mEI agar and modified mTEC agar membrane filtration method, respectively. Twenty-seven enterococci results from the MPN method, one enterococci result from the mEI method, and two E. coli results from the modified mTEC method, were less than detection.

Table 5 lists the USEPA recreational criteria for the protection of public health for single-sample densities of enterococci and E. coli (U.S. Environmental Protection Agency, 2004a). Enterococci results from the MPN analyses were compared to criteria in table 5 based on site classifications (table 1) as follows: results at freshwater and mixed sites were compared to the infrequent full body contact criteria of 151 colonies per $100 \mathrm{~mL}$; results at saltwater sites were compared to the infrequent full body contact criteria of 501 colonies per $100 \mathrm{~mL}$; and results at beach sites were compared to the designated beach area criteria of 104 colonies per $100 \mathrm{~mL}$ (table 5). Only the enterococci results from the MPN method were compared to USEPA criteria in this report because the MPN analyses were considered the primary method for analyses by MDEQ for this study. Of the 200 enterococci samples analyzed by the MPN method, 162 results were less than the USEPA criteria (table 4). Seventeen samples from sites considered to be freshwater or mixed exceeded the criterion, ranging from 162 to 719 MPN per $100 \mathrm{~mL}$; 5 samples from sites considered to be saltwater exceeded the criterion, ranging from 644 to 24,200 MPN per $100 \mathrm{~mL}$; and 16 samples from designated beach sites exceeded the criterion, ranging from 109 to 1,918 MPN per $100 \mathrm{~mL}$. The highest density of 24,200 MPN per $100 \mathrm{~mL}$ occurred at Bayou Chico at Pascagoula, MS, on October 11, 2005 (table 4). Densities at most of the bacteriological sites increased during the second week of sampling, possibly due 
to runoff from an estimated 2-day rainfall total of about 3 inches, caused by outer bands of Hurricane Rita, which made landfall along the western Louisiana and eastern Texas coastline, September 23-24, 2005 (National Oceanic and Atmospheric Administration, 2005b).

Thirty samples from all site types were analyzed for E. coli using the modified mTEC membrane filtration method during the study period (table 4). Of the 30 samples, 13 samples were reported as estimated due to non-ideal colony counts. The highest E. coli density was 2,400 CFU per $100 \mathrm{~mL}$ at the Mississippi Sound at Maywood Road at Gulfport, MS, on October 18, 2005. Because criteria for E. coli are only available for freshwaters (U.S. Environmental Protection Agency, 2004a), densities from freshwater and mixed sites for this study were compared to criteria listed in table 5, specifically, to the single-sample density of $575 \mathrm{CFU}$ per $100 \mathrm{~mL}$ for infrequent full-body contact. Of the $13 \mathrm{E}$. coli samples from freshwater or mixed sites, none exceeded the criterion.

Eleven duplicate, 11 procedure blank, 1 field blank, 5 positive control, and 5 negative control quality-assurance samples were run using the MPN Enterolert method during the study period (table 6). Relative percent differences (RPD) were calculated for the 11 duplicate samples by subtracting the density of the duplicate from the environmental sample, dividing by their average, and multiplying by 100 (RPDs not shown). The average RPD was 49 percent, and ranged from 0 to 152 percent. There were no detections of enterococci in the procedure blanks. The field blank had a density of $8 \mathrm{MPN}$ per $100 \mathrm{~mL}$; although this density was slightly above detection, it did not reflect potential contamination to the environmental results due to improper handling by field personnel. All of the positive control samples were greater than 2,420 MPN per $100 \mathrm{~mL}$, and all of the negative control samples were less than detection, as expected.

\section{Water-Quality Results}

Nineteen water-quality samples were collected at 12 sites over a 2-week period beginning September 19, 2005 (table 2). Water measurements and water-quality analysis for samples collected during the study period included the following constituents and compounds as listed in table 7 with their respective reporting levels:

- Physical properties and other water-quality measurements - discharge (flow), turbidity, dissolved oxygen, $\mathrm{pH}$, specific conductance, and temperature;

- Nitrogen, phosphorus, carbon, and oxygen demand - 5 nitrogen and 3 phosphorus compounds, total organic carbon, biochemical oxygen demand, and chemical oxygen demand;

- 34 major ions, trace metals, and related constituents - including residue on evaporation (total dissolved solids), alkalinity, and bicarbonate;

- 82 modern-use pesticide compounds and degradation products;

- 60 polar pesticide compounds and degradation products;

- 63 waste-water organic compounds and degradation products; and

- 85 volatile organic compounds and degradation products.

Organic compounds listed in table 7 are grouped according to standardized NWQL laboratory schedules; selected compounds may be repeated in other lists. For example, metalaxyl is listed in both the polar pesticide and wastewater compound lists in table 7. There were 265 constituents and compounds that were not detected in any of the samples (compounds that are listed in bold in table 7). Concentrations of constituents and compounds that were detected at each site and water-quality criteria for freshwater systems, where available, are listed in table 8 . Nearly all of the constituents and compounds detected were below water-quality criteria except for the following:

- Dissolved oxygen concentrations were 3.3 and 3.6 milligrams per liter (mg/L) at Tchoutacabouffa River at D'Iberville, MS, on September 21 and 28, respectively. Dissolved oxygen was $2.3 \mathrm{mg} / \mathrm{L}$ at Jourdan River near Bay St. Louis, MS, on September 28. The State of Mississippi dissolved oxygen criterion is $5 \mathrm{mg} / \mathrm{L}$ (4 mg/L instantaneous) (State of Mississippi, 2003).

- Total phosphorus was $0.137 \mathrm{mg} / \mathrm{L}$ at the Leaf River near McClain, MS, on September 27. The USEPA recommends that total phosphorus not exceed $0.1 \mathrm{mg} / \mathrm{L}$ for flowing water that does not discharge into a lake or reservoir (U.S. Environmental Protection Agency, 1986).

- Aluminum concentrations in 11 samples exceeded the USEPA criteria continuous concentration (similar to chronic toxicity) of 87 micrograms per liter $(\mu \mathrm{g} / \mathrm{L})$, but none exceeded the criteria maximum concentration (similar to acute toxicity) of 750 $\mu \mathrm{g} / \mathrm{L}$ (U.S. Environmental Protection Agency, 2004b). Aluminum is considered a non-priority pollutant.

- Copper concentrations were $13.1 \mu \mathrm{g} / \mathrm{L}$ at Bluff Creek at Vancleave, MS, on September 27 and $5 \mu \mathrm{g} / \mathrm{L}$ at Jourdan River near Bay St. Louis, MS, on September 21. The acute and chronic water-quality criteria for State of Mississippi waters are 7 and 5 $\mu \mathrm{g} / \mathrm{L}$, respectively (State of Mississippi, 2003). Copper is considered a priority pollutant. 
It should be noted that there were about 18 detections of organic chemicals above respective detection limits (there were also compounds that were detected, but concentrations were estimated below detection limits). Detections included the presence of caffeine, toluene, N,N-diethyl-meta-toluamide (DEET), naphthalene, and para-nonylphenol, which are wastewater organic compounds. These detections indicated the presence of wastewater at selected sampling locations; however, concentrations were very low, and none exceeded available water-quality criteria.

Quality-assurance samples were used to assess bias and variability in the water-quality data (table 9). All of the equipment blank constituents and compounds were either below detection or were estimated below the laboratory reporting level, except for ammonia plus organic nitrogen (filtered), which had a concentration of $0.16 \mathrm{mg} / \mathrm{L}$. There were several significant detections in the field blank at Red Creek at Vestry, MS, on September 29, especially for chemical oxygen demand and for several major ions and trace metals. Although every precaution was taken in the field to ensure a clean working environment, the samples were collected and processed under adverse conditions, and consequently, some contamination may have occurred. Therefore, the reader is cautioned in interpreting results for the following constituents as there may have been some bias that was introduced during sample collection and processing: chemical oxygen demand, sodium, chloride, silica, barium, calcium, lead, nickel, and zinc.

A duplicate sample was collected at Cypress Creek near Janice, MS, and RPDs were computed for detectable constituents. All RPDs were lower than 20 percent, signifying that variability associated with random errors in the collection, processing, and analysis of the samples was minimal (RPDs not shown). Results from laboratory spike samples indicated that the laboratory could successfully detect constituents when they were present, matrix effects were minimal, and little degradation occurred in sample processing, shipping, and storage (data not shown).

\section{SUMMARY}

Nearly all bacteriological results were below current criteria, and several were below detection; bacteriological results above current criteria were isolated, localized, and were sporadic, for the most part, with the exception of several occurrences possibly due to rainfall from Hurricane Rita, September 23-24, 2005. Nearly all of the results from the water-quality samples were below available criteria, with the exceptions of a few dissolved oxygen, total phosphorus, aluminum, and copper concentrations. Although a few wastewater compounds were detected at selected sites, concentrations were very low, and none exceeded available criteria. Therefore, it did not appear that there was any systematic contamination in the aftermath of Hurricane Katrina at the streams sampled during this study.

\section{ACKNOWLEDGMENTS}

The authors of this report wish to acknowledge the contributions of laboratory and field personnel involved in the bacteriological and water-quality sampling for this project. Personnel from the USGS Mississippi Water Science Center are numerous and are not listed here, but the authors wish to acknowledge from the USGS, Callie Oblinger, Southeastern Regional Water-Quality Specialist from Raleigh, North Carolina, who helped establish and operate the temporary bacteria lab; and Richard Moreland, from the Alabama Water Science Center in Montgomery, Alabama, who helped collect water-quality samples the week of September 19, 2005. The authors also wish to acknowledge MDEQ field personnel Emily Cotton, Tony Cox, Jeff Ethridge, and Ken Dollar, and laboratory personnel, Jennifer Milner, Alice Dossett, Otis Clark, and Kathy Farris for their contributions to the project. Together, all of the laboratory and field personnel from both agencies worked tirelessly for long hours under difficult circumstances. 


\section{REFERENCES}

Francy, D.S., Bushon, R.N., Brady, A.M.G., Kephart, C.M., and Stoeckel, D.M., 2005, Quality-assurance and quality-control manual for the Ohio Water Microbiology Laboratory, accessed October 19, 2005, at

IDEXX Corporation, 2005a, Quanti-Tray/2000® insert and MPN table, accessed November 29, 2005, at http://www.idexx.com/water/refs/060232007.pdf

IDEXX Corporation, 2005b, IDEXX Quanti-Tray/2000® MPN table (per $100 \mathrm{~mL}$ ) with $95 \%$ confidence intervals, accessed November 29, 2005, at http://www.idexx.com/water/refs/qt2k95.pdf

Mississippi Department of Environmental Quality, 2005, Mississippi beach monitoring program, accessed November 29, 2005, at http://www.usm.edu/gcrl/msbeach/index.cgi

Myers, D.N. and Wilde, F.D., eds., November 2004, Biological indicators (3d ed.): U.S. Geological Survey Techniques of WaterResources Investigations, book 9, chap. A7.1, accessed October 20, 2005, at http://pubs.water.usgs.gov/twri9A7/

National Oceanic and Atmospheric Administration, 2005a, National status and trends program - integrated response to Hurricane Katrina: Center for Coastal Monitoring and Assessment, accessed November 29, 2005, at http://ccma.nos.noaa.gov/cit/katrina/

National Oceanic and Atmospheric Administration, 2005b, National Weather Service precipitation analysis for September 23-24, 2005, accessed February 2, 2006, at http://www.srh.noaa.gov/rfcshare/precip_analysis.php

State of Mississippi, 2003, Water quality criteria for intrastate, interstate, and coastal waters - State of Mississippi, Mississippi Department of Environmental Quality, Office of Pollution Control, accessed December 23, 2005, at http://www.deq.state.ms.us/newweb/MDEQRegulations.nsf/RN/WPC-2

U.S. Environmental Protection Agency, 1986, Quality criteria for water - 1986, U.S. Environmental Protection Agency, Office of Water Regulations and Standards, Washington D.C., May 1, 1986, EPA 440/5-86-001, accessed December 23, 2005, at http://www.epa.gov/waterscience/criteria/goldbook.pdf

U.S. Environmental Protection Agency, 1999, 1999 update of ambient water quality criteria for ammonia, U.S. Environmental Protection Agency, Office of Water, December 1999, EPA-822-R-99-014, accessed on December 23, 2005, at http://www.epa.gov/waterscience/criteria/ammonia/99update.pdf

U.S. Environmental Protection Agency, 2000, Improved enumeration methods for the recreational water quality indicators - enterococci and Escherichia coli: Washington, D.C., Office of Science and Technology, EPA/821/R-97/004, 49 p.

U.S. Environmental Protection Agency, 2003a, Guidelines establishing test procedures for the analysis of pollutants - analytical methods for biological pollutants in ambient water - final rule: Washington, D.C., Federal Register, v. 68, no. 139, July 21, 2003, p. $43272-43283$.

U.S. Environmental Protection Agency, 2003b, Notice of Revised Draft Ambient Water Quality Criteria Document for Atrazine and Request for Scientific Views, U.S. Environmental Protection Agency Fact Sheet, November 2003, accessed on December 23, 2005, at http://www.epa.gov/waterscience/criteria/atrazine/atrazinefacts.html

U.S. Environmental Protection Agency, 2003c, Notice of Availability of Draft Diazinon Criteria Document and Request for Scientific Views, U.S. Environmental Protection Agency Fact Sheet, December 2003, accessed on December 23, 2005, at http://www.epa.gov/waterscience/criteria/diazinon/draft-fs.htm

U.S. Environmental Protection Agency, 2004a, Water quality standards for coastal and great lakes recreation waters: Washington, D.C., Federal Register, v. 69, no. 220, November, 16, 2004, p. 67217-67243.

U.S. Environmental Protection Agency, 2004b, National recommended water quality criteria, U.S. Environmental Protection Agency, Office of Water and Office of Science and Technology, 2004, accessed on December 23, 2005, at http://www.epa.gov/waterscience/criteria/nrwqc-2004.pdf

U.S. Environmental Protection Agency, 2005, Water quality study of bays in coastal Mississippi water quality report October 28, 2005, accessed November 29, 2005, at http://www.epa.gov/region4/sesd/reports/2005-0926.html

U.S. Geological Survey, variously dated, National field manual for the collection of water-quality data: U.S. Geological Survey Techniques of Water-Resources Investigations, book 9, chaps. A1-A9, available online at http://pubs.water.usgs.gov/twri9A 
Table 1. Bacteriological sampling sites - Hancock, Harrison, and Jackson Counties, Mississippi - post Hurricane Katrina, September 19 through October 18, 2005

[USGS, U.S. Geological Survey; datum is North American Datum 1983; MS, Mississippi]

\begin{tabular}{|c|c|c|c|c|c|c|}
\hline $\begin{array}{c}\text { Site } \\
\text { identifier } \\
\text { (figures } 1 . \\
\text { 4) }\end{array}$ & $\begin{array}{c}\text { USGS station } \\
\text { number }\end{array}$ & Station name & Site type & Field number & Latitude & Longitude \\
\hline 1 & 02481660 & Jourdan River near Bay St. Louis, MS & Mixed & $\mathrm{HK}-1$ & $30^{\circ} 23^{\prime} 14^{\prime \prime}$ & $89^{\circ} 26^{\prime} 29^{\prime \prime}$ \\
\hline 2 & 02481670 & Bayou La Croix near Clermont Harbour, MS & Saltwater & HK-2 & $30^{\circ} 19^{\prime} 25^{\prime \prime}$ & $89^{\circ} 29^{\prime} 17^{\prime \prime}$ \\
\hline 3 & 02481671 & Bayou La Croix at State Highway 603 near Waveland, MS & Saltwater & HK-3 & $30^{\circ} 19^{\prime} 48^{\prime \prime}$ & $89^{\circ} 25^{\prime} 29^{\prime \prime}$ \\
\hline 4 & 02492610 & Turtleskin Creek near Santa Rosa, MS & Freshwater & HK-4 & $30^{\circ} 24^{\prime} 24^{\prime \prime}$ & $89^{\circ} 38^{\prime} 01^{\prime \prime}$ \\
\hline 5 & 02481565 & Wolf Branch near Santa Rosa, MS & Freshwater & HK-5 & $30^{\circ} 24^{\prime} 34^{\prime \prime}$ & $89^{\circ} 32^{\prime} 57^{\prime \prime}$ \\
\hline 6 & 02481649 & Orphan Creek at Highway 43 near Kiln, MS & Freshwater & HK-6 & $30^{\circ} 27^{\prime} 16^{\prime \prime}$ & $89^{\circ} 28^{\prime} 26^{\prime \prime}$ \\
\hline 7 & 0248166518 & Bayou LaTerra at Fenton, MS & Freshwater & HK-7 & $30^{\circ} 24^{\prime} 51^{\prime \prime}$ & $89^{\circ} 22^{\prime} 57^{\prime \prime}$ \\
\hline 8 & 02481663 & Rotten Bayou near Fenton, MS & Freshwater & HK-8 & $30^{\circ} 25^{\prime} 09^{\prime \prime}$ & $89^{\circ} 20^{\prime} 40^{\prime \prime}$ \\
\hline 9 & 0248166310 & Mill Creek at Fenton, MS & Freshwater & HK-9 & $30^{\circ} 24^{\prime} 34^{\prime \prime}$ & $89^{\circ} 20^{\prime} 40^{\prime \prime}$ \\
\hline 10 & 301541089241355 & Mississippi Sound near Buccaneer State Park near Waveland, MS & Beach & HKB-1 & $30^{\circ} 15^{\prime} 41^{\prime \prime}$ & $89^{\circ} 24^{\prime} 13^{\prime \prime}$ \\
\hline 11 & 301650089215300 & Mississippi Sound at Waveland, MS & Beach & HKB-2 & $30^{\circ} 16^{\prime} 50^{\prime \prime}$ & $89^{\circ} 21^{\prime} 53^{\prime \prime}$ \\
\hline 12 & 301808089193955 & Mississippi Sound at Bay St. Louis, MS & Beach & HKB-3 & $30^{\circ} 18^{\prime} 08^{\prime \prime}$ & $89^{\circ} 19^{\prime} 39^{\prime \prime}$ \\
\hline 13 & 02481524 & Wolf River near Cuevas, MS & Mixed & $\mathrm{HN}-1$ & $30^{\circ} 22^{\prime} 32^{\prime \prime}$ & $89^{\circ} 13^{\prime} 51^{\prime \prime}$ \\
\hline 14 & 02481310 & Bayou Portage at Cuevas, MS & Mixed & $\mathrm{HN}-2$ & $30^{\circ} 21^{\prime} 46^{\prime \prime}$ & $89^{\circ} 13^{\prime} 06^{\prime \prime}$ \\
\hline 15 & 02481526 & DeLisle Bayou near DeLisle, MS & Freshwater & $\mathrm{HN}-3$ & $30^{\circ} 23^{\prime} 37^{\prime \prime}$ & $89^{\circ} 15^{\prime} 40^{\prime \prime}$ \\
\hline 16 & 02481510 & Wolf River near Landon, MS & Freshwater & $\mathrm{HN}-4$ & $30^{\circ} 29^{\prime} 01^{\prime \prime}$ & $89^{\circ} 16^{\prime} 28^{\prime \prime}$ \\
\hline 17 & 02481240 & Turkey Creek (Canal Road) near Gulfport, MS & Freshwater & $\mathrm{HN}-5$ & $30^{\circ} 23^{\prime} 54^{\prime \prime}$ & $89^{\circ} 08^{\prime} 12^{\prime \prime}$ \\
\hline 18 & 02481194 & Bayou Bernard (Canal Road) near Landon, MS & Freshwater & $\mathrm{HN}-6$ & $30^{\circ} 26^{\prime} 39^{\prime \prime}$ & $89^{\circ} 08^{\prime} 13^{\prime \prime}$ \\
\hline 19 & 02481252 & Turkey Creek at Creosote Road near Gulfport, MS & Saltwater & $\mathrm{HN}-7$ & $30^{\circ} 25^{\prime} 27^{\prime \prime}$ & $89^{\circ} 04^{\prime} 14^{\prime \prime}$ \\
\hline 20 & 02481130 & Biloxi River near Lyman, MS & Freshwater & $\mathrm{HN}-8$ & $30^{\circ} 29^{\prime} 18^{\prime \prime}$ & $89^{\circ} 02^{\prime} 09^{\prime \prime}$ \\
\hline 21 & 02480606 & Howard Creek at Cedar Lake, MS & Freshwater & $\mathrm{HN}-9$ & $30^{\circ} 28^{\prime} 06^{\prime \prime}$ & $88^{\circ} 56^{\prime} 18^{\prime \prime}$ \\
\hline 22 & 02480590 & Tchoutacabouffa River near D'Iberville, MS & Freshwater & $\mathrm{HN}-10$ & $30^{\circ} 28^{\prime} 33^{\prime \prime}$ & $88^{\circ} 53^{\prime} 36^{\prime \prime}$ \\
\hline 23 & 02480595 & Cypress Creek near D'Iberville, MS & Freshwater & HN-11 & $30^{\circ} 27^{\prime} 41^{\prime \prime}$ & $88^{\circ} 53^{\prime} 30^{\prime \prime}$ \\
\hline 24 & 301827089173955 & Mississippi Sound at Henderson Point, MS & Beach & HNB-1 & $30^{\circ} 18^{\prime} 27^{\prime \prime}$ & $89^{\circ} 17^{\prime} 39^{\prime \prime}$ \\
\hline 25 & 301843089145255 & Mississippi Sound at Pass Christian Harbour at Pass Christian, MS & Beach & HNB-2 & $30^{\circ} 18^{\prime} 43^{\prime \prime}$ & $89^{\circ} 14^{\prime} 52^{\prime \prime}$ \\
\hline 26 & 302044089083655 & Mississippi Sound at Long Beach, MS & Beach & HNB-3 & $30^{\circ} 20^{\prime} 44^{\prime \prime}$ & $89^{\circ} 08^{\prime} 36^{\prime \prime}$ \\
\hline 27 & 302147089051055 & Mississipi Sound at Gulfport Harbor at Gulfport, MS & Beach & HNB-4 & $30^{\circ} 21^{\prime} 47^{\prime \prime}$ & $89^{\circ} 05^{\prime} 10^{\prime \prime}$ \\
\hline 28 & 302237089025855 & Mississippi Sound at Maywood Road at Gulfport, MS & Beach & HNB-5 & $30^{\circ} 22^{\prime} 37^{\prime \prime}$ & $89^{\circ} 02^{\prime} 58^{\prime \prime}$ \\
\hline 29 & 302328088575655 & Mississippi Sound at Broadwater Marina at Biloxi, MS & Beach & HNB-6 & $30^{\circ} 23^{\prime} 28^{\prime \prime}$ & $88^{\circ} 57^{\prime} 56^{\prime \prime}$ \\
\hline 30 & 302318088512600 & Biloxi Bay at Point Cadet Harbor at Biloxi, MS & Beach & HNB-7 & $30^{\circ} 23^{\prime} 18^{\prime \prime}$ & $88^{\circ} 51^{\prime} 26^{\prime \prime}$ \\
\hline 31 & 02481299 & Old Fort Bayou at Ocean Springs, MS & Saltwater & $\mathrm{JK}-1$ & $30^{\circ} 25^{\prime} 09^{\prime \prime}$ & $88^{\circ} 49^{\prime} 41^{\prime \prime}$ \\
\hline 32 & 02481292 & Perigal Creek near D'Iberville, MS & Freshwater & $\mathrm{JK}-2$ & $30^{\circ} 28^{\prime} 58^{\prime \prime}$ & $88^{\circ} 49^{\prime} 49^{\prime \prime}$ \\
\hline 33 & 0248129250 & South Branch Perigal Creek near Ocean Springs, MS & Freshwater & $\mathrm{JK}-3$ & $30^{\circ} 27^{\prime} 52^{\prime \prime}$ & $88^{\circ} 46^{\prime} 17^{\prime \prime}$ \\
\hline 34 & 02480289 & Davis Bayou at Ocean Springs, MS & Saltwater & $\mathrm{JK}-4$ & $30^{\circ} 23^{\prime} 21^{\prime \prime}$ & $88^{\circ} 47^{\prime} 31^{\prime \prime}$ \\
\hline 35 & 02480288 & Simmons Bayou at Ocean Springs, MS & Saltwater & JK-5 & $30^{\circ} 22^{\prime} 28^{\prime \prime}$ & $88^{\circ} 45^{\prime} 35^{\prime \prime}$ \\
\hline 36 & 02480284 & Mary Walker Bayou at Gautier, MS & Mixed & JK-6 & $30^{\circ} 24^{\prime} 38^{\prime \prime}$ & $88^{\circ} 39^{\prime} 18^{\prime \prime}$ \\
\hline 37 & 02480286 & Bayou St. Pierre at Gautier, MS & Saltwater & JK-7 & $30^{\circ} 22^{\prime} 35^{\prime \prime}$ & $88^{\circ} 36^{\prime} 51^{\prime \prime}$ \\
\hline 38 & 02480285 & West Pascagoula at Highway 90 at Gautier, MS & Saltwater & JK-7A & $30^{\circ} 22^{\prime} 58^{\prime \prime}$ & $88^{\circ} 36^{\prime} 32^{\prime \prime}$ \\
\hline 39 & 02480205 & Black Creek near Helena, MS & Freshwater & $\mathrm{JK}-8$ & $30^{\circ} 28^{\prime} 21^{\prime \prime}$ & $88^{\circ} 28^{\prime} 18^{\prime \prime}$ \\
\hline 40 & 0247109250 & Bayou Chico at Pascagoula, MS & Saltwater & JK-9 & $30^{\circ} 21^{\prime} 17^{\prime \prime}$ & $88^{\circ} 31^{\prime} 36^{\prime \prime}$ \\
\hline 41 & 0247109160 & West Prong Bayou Cassotte at Louise Road at Pascagoula, MS & Saltwater & $\mathrm{JK}-10$ & $30^{\circ} 21^{\prime} 51^{\prime \prime}$ & $88^{\circ} 30^{\prime} 36^{\prime \prime}$ \\
\hline 42 & 0247109150 & Bayou Cassotte at Orchard Avenue at Pascagoula, MS & Saltwater & $\mathrm{JK}-11$ & $30^{\circ} 22^{\prime} 05^{\prime \prime}$ & $88^{\circ} 30^{\prime} 05^{\prime \prime}$ \\
\hline 43 & 302421088495255 & Biloxi Bay at Jackson Avenue at Ocean Springs, MS & Beach & JKB-1 & $30^{\circ} 24^{\prime} 21^{\prime \prime}$ & $88^{\circ} 49^{\prime} 52^{\prime \prime}$ \\
\hline 44 & 302036088320955 & Mississippi Sound at Country Club Drive at Pascagoula, MS & Beach & JKB-2 & $30^{\circ} 20^{\prime} 36^{\prime \prime}$ & $88^{\circ} 32^{\prime} 09^{\prime \prime}$ \\
\hline 45 & 302031088313855 & Mississippi Sound near Chico Bayou at Pascagoula, MS & Beach & JKB-3 & $30^{\circ} 20^{\prime} 31^{\prime \prime}$ & $88^{\circ} 31^{\prime} 38^{\prime \prime}$ \\
\hline
\end{tabular}


Table 2. Surface water-quality sampling sites, southeastern Mississippi, post-Hurricane Katrina, September 19-30, 2005.

[Datum is North American Datum 1983; MS, Mississippi; RT, active real-time gage; ---, did not sample; CSG, active crest-stage gage]

\begin{tabular}{|c|c|c|c|c|c|c|c|c|}
\hline $\begin{array}{c}\text { Site } \\
\text { identifier } \\
\text { (figure 5) }\end{array}$ & $\begin{array}{l}\text { Station } \\
\text { number }\end{array}$ & Station name & $\begin{array}{c}\text { Type of } \\
\text { site }\end{array}$ & $\begin{array}{c}\text { Number } \\
\text { of } \\
\text { samples }\end{array}$ & $\begin{array}{c}\text { Week } 1 \\
\text { sampling } \\
\text { date }\end{array}$ & $\begin{array}{c}\text { Week } 2 \\
\text { sampling } \\
\text { date }\end{array}$ & Latitude & Longitude \\
\hline A & 02475000 & Leaf River near McClain, MS & $\mathrm{RT}$ & 1 & --- & $9 / 27 / 2005$ & $31^{\circ} 06^{\prime} 10^{\prime \prime}$ & $88^{\circ} 48^{\prime} 30^{\prime \prime}$ \\
\hline $\mathrm{B}$ & 02478500 & Chickasawhay River at Leakesville, MS & RT & 1 & --- & $9 / 27 / 2005$ & $31^{\circ} 08^{\prime} 55^{\prime \prime}$ & $88^{\circ} 32^{\prime} 53^{\prime \prime}$ \\
\hline $\mathrm{C}$ & 02479155 & Cypress Creek near Janice, MS & $\mathrm{RT}$ & 1 & 9/19/2005 & --- & $31^{\circ} 01^{\prime} 31^{\prime \prime}$ & $89^{\circ} 01^{\prime} 00^{\prime \prime}$ \\
\hline $\mathrm{D}$ & 02479160 & Black Creek near Wiggins, MS & RT & 2 & $9 / 22 / 2005$ & $9 / 29 / 2005$ & $30^{\circ} 51^{\prime} 12^{\prime \prime}$ & $88^{\circ} 54^{\prime} 49^{\prime \prime}$ \\
\hline $\mathrm{E}$ & 02479300 & Red Creek at Vestry, MS & $\mathrm{RT}$ & 2 & 9/20/2005 & 9/29/2005 & $30^{\circ} 44^{\prime} 10^{\prime \prime}$ & $88^{\circ} 46^{\prime} 52^{\prime \prime}$ \\
\hline $\mathrm{F}$ & 02479330 & Pascagoula River at Cumbest Bluff, MS & RT & 1 & --- & 9/27/2005 & $30^{\circ} 35^{\prime} 02^{\prime \prime}$ & $88^{\circ} 34^{\prime} 12^{\prime \prime}$ \\
\hline G & 02479560 & Escatawpa River near Agricola, MS & RT & 1 & 9/20/2005 & --- & $30^{\circ} 48^{\prime} 12^{\prime \prime}$ & $88^{\circ} 27^{\prime} 31^{\prime \prime}$ \\
\hline $\mathrm{H}$ & 02480254 & Bluff Creek at Vancleave, MS & RT & 2 & $9 / 20 / 2005$ & 9/27/2005 & $30^{\circ} 31^{\prime} 55^{\prime \prime}$ & $88^{\circ} 41^{\prime} 25^{\prime \prime}$ \\
\hline I & 02480599 & Tchoutacabouffa River at D'Iberville, MS & CSG & 2 & $9 / 21 / 2005$ & $9 / 28 / 2005$ & $30^{\circ} 27^{\prime} 36^{\prime \prime}$ & $88^{\circ} 54^{\prime} 03^{\prime \prime}$ \\
\hline $\mathrm{J}$ & 02481000 & Biloxi River at Wortham, MS & $\mathrm{RT}$ & 2 & 9/19/2005 & 9/28/2005 & $30^{\circ} 33^{\prime} 31^{\prime \prime}$ & $89^{\circ} 07^{\prime} 19^{\prime \prime}$ \\
\hline $\mathrm{K}$ & 02481510 & Wolf River near Landon, MS & RT & 2 & $9 / 21 / 2005$ & $9 / 28 / 2005$ & $30^{\circ} 29^{\prime} 01^{\prime \prime}$ & $89^{\circ} 16^{\prime} 28^{\prime \prime}$ \\
\hline $\mathrm{L}$ & 02481660 & Jourdan River near Bay St. Louis, MS & $\mathrm{RT}$ & 2 & $9 / 21 / 2005$ & $9 / 28 / 2005$ & $30^{\circ} 23^{\prime} 14^{\prime \prime}$ & $89^{\circ} 26^{\prime} 29^{\prime \prime}$ \\
\hline
\end{tabular}


Table 3. Bottle requirements, filtering requirements, and sample-preservation techniques for waterquality samples collected in the aftermath of Hurricane Katrina, September through October 2005.

[mL, millilter; $\mu \mathrm{m}$, micrometer; C, Celsius; L, liter; <, less than; $\mathrm{HCl}$, hydrochloric acid]

\begin{tabular}{|c|c|c|c|c|}
\hline $\begin{array}{l}\text { Type of sample (number of bottles } \\
\text { required) }\end{array}$ & Bottles required & Filtered or unfiltered & Filter type & Sample preservation \\
\hline \multirow[t]{2}{*}{ Nutrients (2) } & $\begin{array}{l}\text { 125-mL brown } \\
\text { polypropylene }\end{array}$ & Filtered & $0.45 \mu \mathrm{m}$ cartridge-type filter & Chilled at $4^{\circ} \mathrm{C}$ \\
\hline & $\begin{array}{l}125-\mathrm{mL} \text { translucent } \\
\text { polypropylene }\end{array}$ & Unfiltered & None & $\begin{array}{l}\text { Acidified with } 1 \mathrm{~mL} \text { of } 4.5 \text { normal } \\
\text { sulfuric acid; then chilled at } 4^{\circ} \mathrm{C}\end{array}$ \\
\hline Total organic carbon (1) & 125-baked amber glass & Unfiltered & None & Chilled at $4^{\circ} \mathrm{C}$ \\
\hline Chemical oxygen demand (1) & 125-baked amber glass & Unfiltered & None & $\begin{array}{l}\text { Acidified with sulfuric acid to a } \\
\mathrm{pH} \text { of }<2 \text {; chilled at } 4^{\circ} \mathrm{C}\end{array}$ \\
\hline Biochemical oxygen demand & $\begin{array}{l}\text { 1-L translucent } \\
\text { polypropylene }\end{array}$ & Unfiltered & None & Chilled at $4^{\circ} \mathrm{C}$ \\
\hline \multirow[t]{3}{*}{ Major Ions (3) } & $\begin{array}{l}250-m L \text { polypropylene, acid- } \\
\text { rinsed }\end{array}$ & Filtered & $0.45 \mu \mathrm{m}$ cartridge-type filter & $\begin{array}{l}\text { Acidified with nitric acid to a } \mathrm{pH} \\
\text { of }<2\end{array}$ \\
\hline & 250-mL polypropylene & Filtered & $0.45 \mu \mathrm{m}$ cartridge-type filter & None \\
\hline & 250-mL polypropylene & Unfiltered & None & None \\
\hline Trace metals (2) & $\begin{array}{l}250-\mathrm{mL} \text { polypropylene, acid- } \\
\text { rinsed } \\
250-\mathrm{mL} \text { polypropylene }\end{array}$ & $\begin{array}{l}\text { Filtered } \\
\text { Unfiltered }\end{array}$ & $\begin{array}{l}0.45 \mu \mathrm{m} \text { cartridge-type filter } \\
\text { None }\end{array}$ & $\begin{array}{l}\text { Acidified with nitric acid to a } \mathrm{pH} \\
\text { of }<2 \\
\text { None }\end{array}$ \\
\hline Modern-use pesticides (1) & 1-L baked amber glass & Filtered & $0.7 \mu \mathrm{m}$ glass fiber filter & Chilled at $4^{\circ} \mathrm{C}$ \\
\hline Polar pesticides (1) & 1-L baked amber glass & Filtered & $0.7 \mu \mathrm{m}$ glass fiber filter & Chilled at $4^{\circ} \mathrm{C}$ \\
\hline Waste water organic compounds (1) & 1-L baked amber glass & Filtered & $0.7 \mu \mathrm{m}$ glass fiber filter & Chilled at $4^{\circ} \mathrm{C}$ \\
\hline Volatile organic compounds (3) & 40-mL amber glass bottle & Unfiltered & None & $\begin{array}{l}\text { Bottle is filled completely with } \\
\text { sample; sample is adjusted with } \\
\text { a } 1: 1 \mathrm{HCl} / \text { water solution to a pH } \\
\text { of }<2 \text {; sample is protected from } \\
\text { sunlight and chilled at } 4^{\circ} \mathrm{C}\end{array}$ \\
\hline
\end{tabular}


Table 4. Results of bacteriological analyses for water samples collected in the aftermath of Hurricane Katrina, coastal Mississippi Counties, September 19 - October 18, 2005

[MPN, most probable number; $\mathrm{mL}$, milliliters; CFU, colony-forming units; numbers in brackets are unique field numbers; numbers in parenthesis are 95 percent confidence intervals; <, less than; E, estimated due to non-ideal colony counts]

\begin{tabular}{|c|c|c|c|c|}
\hline Sample date & Sample time & $\begin{array}{c}\text { Enterococci } \\
\text { densities analyzed } \\
\text { by Enterolert most } \\
\text { probable number } \\
\text { method, } \\
\text { MPN per } 100 \mathrm{~mL}\end{array}$ & $\begin{array}{c}\text { Enterococci } \\
\text { densities analyzed } \\
\text { by membrane } \\
\text { filtration, mEl agar } \\
\text { method, } \\
\text { CFU per } 100 \mathrm{~mL} \\
\end{array}$ & $\begin{array}{c}\text { Escherichia Coli } \\
\text { densities analyzed } \\
\text { by membrane } \\
\text { filtration, modified } \\
\text { mTEC agar method, } \\
\text { CFU per } 100 \mathrm{~mL}\end{array}$ \\
\hline \multicolumn{5}{|c|}{02481660 Jourdan River near Bay St. Louis, MS [HK-1] } \\
\hline $9 / 20 / 2005$ & 0925 & $37(25-53)$ & & \\
\hline 9/27/2005 & 0910 & $79(56-109)$ & & \\
\hline $10 / 4 / 2005$ & 1200 & $26(17-38)$ & & \\
\hline 10/11/2005 & 1710 & $189(142-251)$ & & \\
\hline $10 / 17 / 2005$ & 1550 & $<10(0-37)$ & & \\
\hline \multicolumn{5}{|c|}{02481670 Bayou La Croix near Clermont Harbour, MS [HK-2] } \\
\hline 9/20/2005 & 1125 & $40(11-89)$ & & \\
\hline 9/27/2005 & 1245 & $712(507-983)$ & & \\
\hline $10 / 4 / 2005$ & 1420 & $52(18-108)$ & & \\
\hline 10/11/2005 & 1730 & $<10(0-37)$ & & \\
\hline 10/17/2005 & 1615 & $<10(0-37)$ & $10 \mathrm{E}$ & $25 \mathrm{E}$ \\
\hline \multicolumn{5}{|c|}{02481671 Bayou La Croix at State Highway 603 near Waveland, MS [HK-3] } \\
\hline 9/20/2005 & 1100 & $201(124-318)$ & & \\
\hline 9/27/2005 & 1058 & $20(3-59)$ & & \\
\hline $10 / 4 / 2005$ & 1615 & $52(18-108)$ & & \\
\hline 10/11/2005 & 1750 & $213(127-326)$ & & \\
\hline 10/17/2005 & 1630 & $31(7-89)$ & 24E & 44E \\
\hline \multicolumn{5}{|c|}{02492610 Turtleskin Creek near Santa Rosa, MS [HK-4] } \\
\hline 9/20/2005 & 1005 & $54(39-73)$ & & \\
\hline $9 / 27 / 2005$ & 0957 & $26(16-39)$ & $40 \mathrm{E}$ & $22 \mathrm{E}$ \\
\hline $10 / 4 / 2005$ & 1300 & $4(2-10)$ & & \\
\hline $10 / 11 / 2005$ & 1815 & $24(16-36)$ & & \\
\hline 10/17/2005 & 1700 & $1(0-6)$ & & \\
\hline \multicolumn{5}{|c|}{02481565 Wolf Branch near Santa Rosa, MS [HK-5] } \\
\hline 9/20/2005 & 0945 & $10(4-17)$ & & \\
\hline $9 / 27 / 2005$ & 0935 & $16(9-25)$ & & \\
\hline $10 / 4 / 2005$ & 1245 & $5(2-12)$ & & \\
\hline \multicolumn{5}{|c|}{02481649 Orphan Creek at Highway 43 near Kiln, MS [HK-6] } \\
\hline 9/20/2005 & 0900 & $38(26-54)$ & & \\
\hline 9/27/2005 & 0852 & $75(53-103)$ & & \\
\hline
\end{tabular}


Table 4. Continued--Results of bacteriological analyses for water samples collected in the aftermath of Hurricane Katrina, coastal Mississippi Counties, September 19 - October 18, 2005

\begin{tabular}{|c|c|c|c|c|}
\hline Sample date & Sample time & $\begin{array}{c}\text { Enterococci } \\
\text { densities analyzed } \\
\text { by Enterolert most } \\
\text { probable number } \\
\text { method, } \\
\text { MPN per } 100 \mathrm{~mL} \\
\end{array}$ & $\begin{array}{c}\text { Enterococci } \\
\text { densities analyzed } \\
\text { by membrane } \\
\text { filtration, mEl agar } \\
\text { method, } \\
\text { CFU per } 100 \mathrm{~mL} \\
\end{array}$ & $\begin{array}{c}\text { Escherichia Coli } \\
\text { densities analyzed } \\
\text { by membrane } \\
\text { filtration, modified } \\
\text { mTEC agar method, } \\
\text { CFU per } 100 \mathrm{~mL} \\
\end{array}$ \\
\hline $10 / 4 / 2005$ & 1145 & $6(3-14)$ & & \\
\hline $10 / 11 / 2005$ & 1640 & $16(9-26)$ & & \\
\hline 10/17/2005 & 1530 & $6(3-14)$ & & \\
\hline \multicolumn{5}{|c|}{0248166518 Bayou LaTerra at Fenton, MS [HK-7] } \\
\hline 9/20/2005 & 0837 & $138(98-192)$ & & \\
\hline 9/27/2005 & 0825 & $167(123-223)$ & & \\
\hline $10 / 4 / 2005$ & 1125 & $21(13-33)$ & 143 & 73 \\
\hline 10/11/2005 & 1620 & $25(16-37)$ & & \\
\hline $10 / 17 / 2005$ & 1505 & $8(4-16)$ & & \\
\hline \multicolumn{5}{|c|}{02481663 Rotten Bayou near Fenton, MS [HK-8] } \\
\hline 9/20/2005 & 0830 & $40(27-56)$ & & \\
\hline 9/27/2005 & 0745 & $35(24-50)$ & & \\
\hline $10 / 4 / 2005$ & 1115 & $12(6-20)$ & 43E & 90 \\
\hline $10 / 11 / 2005$ & 1610 & $38(26-54)$ & $56 \mathrm{E}$ & 93 \\
\hline 10/17/2005 & 1455 & $11(5-18)$ & & \\
\hline \multicolumn{5}{|c|}{0248166310 Mill Creek at Fenton, MS [HK-9] } \\
\hline 9/20/2005 & 0815 & $46(32-64)$ & & \\
\hline 9/27/2005 & 0735 & $91(66-123)$ & 168 & $49 \mathrm{E}$ \\
\hline $10 / 4 / 2005$ & 1100 & $32(21-46)$ & & \\
\hline $10 / 11 / 2005$ & 1600 & $20(12-30)$ & & \\
\hline $10 / 17 / 2005$ & 1445 & $31(7-89)$ & & \\
\hline \multicolumn{5}{|c|}{301541089241355 Mississippi Sound near Buccaneer State Park near Waveland, MS [HKB-1] } \\
\hline 9/20/2005 & 1150 & $<10(0-37)$ & & \\
\hline 9/27/2005 & 1130 & $52(23-119)$ & $60 \mathrm{E}$ & 17E \\
\hline $10 / 4 / 2005$ & 1545 & $269(176-391)$ & 198 & $28 \mathrm{E}$ \\
\hline $10 / 12 / 2005$ & 1500 & $<10(0-37)$ & & \\
\hline 10/18/2005 & 1510 & $<10(0-37)$ & & \\
\hline \multicolumn{5}{|c|}{301650089215300 Mississippi Sound at Waveland, MS [HKB-2] } \\
\hline 9/20/2005 & 1215 & $20(3-71)$ & & \\
\hline 9/27/2005 & 1140 & 233 (144-361) & & \\
\hline $10 / 4 / 2005$ & 1530 & $40(11-89)$ & $143 E$ & $49 E$ \\
\hline 10/12/2005 & 1445 & $41(12-91)$ & 70 & 100 \\
\hline 10/18/2005 & 1435 & $10(1-55)$ & & \\
\hline
\end{tabular}


Table 4. Continued--Results of bacteriological analyses for water samples collected in the aftermath of Hurricane Katrina, coastal Mississippi Counties, September 19 - October 18, 2005

\begin{tabular}{|c|c|c|c|c|}
\hline Sample date & Sample time & $\begin{array}{c}\text { Enterococci } \\
\text { densities analyzed } \\
\text { by Enterolert most } \\
\text { probable number } \\
\text { method, } \\
\text { MPN per } 100 \mathrm{~mL} \\
\end{array}$ & $\begin{array}{c}\text { Enterococci } \\
\text { densities analyzed } \\
\text { by membrane } \\
\text { filtration, mEl agar } \\
\text { method, } \\
\text { CFU per } 100 \mathrm{~mL} \\
\end{array}$ & $\begin{array}{c}\text { Escherichia Coli } \\
\text { densities analyzed } \\
\text { by membrane } \\
\text { filtration, modified } \\
\text { mTEC agar method, } \\
\text { CFU per } 100 \mathrm{~mL} \\
\end{array}$ \\
\hline \multicolumn{5}{|c|}{301808089193955 Mississippi Sound at Bay St. Louis, MS [HKB-3] } \\
\hline 9/20/2005 & 1230 & $148(85-251)$ & & \\
\hline 9/27/2005 & 1150 & $20(3-71)$ & & \\
\hline $10 / 4 / 2005$ & 1515 & $<10(0-37)$ & & \\
\hline $10 / 12 / 2005$ & 1525 & $31(7-89)$ & & \\
\hline 10/18/2005 & 1450 & $10(1-55)$ & & \\
\hline \multicolumn{5}{|c|}{02481524 Wolf River near Cuevas, MS [HN-1] } \\
\hline 9/19/2005 & 1750 & $162(115-221)$ & & \\
\hline 9/26/2005 & 1505 & $45(31-61)$ & 180 & 90 \\
\hline 10/4/2005 & 0945 & $62(24-122)$ & & \\
\hline 10/12/2005 & 1200 & $10(1-55)$ & $40 E$ & $51 \mathrm{E}$ \\
\hline $10 / 18 / 2005$ & 1245 & $63(25-127)$ & & \\
\hline \multicolumn{5}{|c|}{02481310 Bayou Portage at Cuevas, MS [HN-2] } \\
\hline 9/19/2005 & 1730 & $91(67-121)$ & & \\
\hline 9/26/2005 & 1458 & $206(147-283)$ & & \\
\hline $10 / 3 / 2005$ & 1430 & $238(146-358)$ & 410 & 120 \\
\hline $10 / 12 / 2005$ & 1155 & $169(94-270)$ & & \\
\hline $10 / 18 / 2005$ & 1235 & $41(17-95)$ & & \\
\hline \multicolumn{5}{|c|}{02481526 DeLisle Bayou near DeLisle, MS [HN-3] } \\
\hline 9/19/2005 & 1818 & $42(28-60)$ & & \\
\hline 9/26/2005 & 1542 & $51(36-69)$ & & \\
\hline $10 / 4 / 2005$ & 1020 & $313(217-440)$ & & \\
\hline $10 / 12 / 2005$ & 1210 & $326(270-388)$ & & \\
\hline $10 / 18 / 2005$ & 1300 & $184(149-222)$ & 182 & $20 \mathrm{E}$ \\
\hline \multicolumn{5}{|c|}{02481510 Wolf River near Landon, MS [HN-4] } \\
\hline 9/20/2005 & 0800 & $122(87-167)$ & & \\
\hline 9/27/2005 & 0802 & $93(68-123)$ & & \\
\hline $10 / 4 / 2005$ & 1040 & $15(8-25)$ & & \\
\hline 10/11/2005 & 1540 & $5(2-12)$ & & \\
\hline $10 / 17 / 2005$ & 1420 & $2(0-7)$ & & \\
\hline \multicolumn{5}{|c|}{02481240 Turkey Creek (Canal Road) near Gulfport, MS [HN-5] } \\
\hline 9/19/2005 & 1542 & 70 (50-95) & & \\
\hline 9/26/2005 & 1410 & $12(6-21)$ & 44E & $18 \mathrm{E}$ \\
\hline 10/3/2005 & 1400 & $58(42-78)$ & & \\
\hline 10/12/2005 & 1655 & 719 (526-946) & $46 \mathrm{E}$ & \\
\hline 10/17/2005 & 1345 & $6(2-12)$ & & \\
\hline
\end{tabular}


Table 4. Continued--Results of bacteriological analyses for water samples collected in the aftermath of Hurricane Katrina, coastal Mississippi Counties, September 19 - October 18, 2005

\begin{tabular}{|c|c|c|c|c|}
\hline Sample date & Sample time & $\begin{array}{c}\text { Enterococci } \\
\text { densities analyzed } \\
\text { by Enterolert most } \\
\text { probable number } \\
\text { method, } \\
\text { MPN per } 100 \mathrm{~mL} \\
\end{array}$ & $\begin{array}{c}\text { Enterococci } \\
\text { densities analyzed } \\
\text { by membrane } \\
\text { filtration, mEl agar } \\
\text { method, } \\
\text { CFU per } 100 \mathrm{~mL} \\
\end{array}$ & $\begin{array}{c}\text { Escherichia Coli } \\
\text { densities analyzed } \\
\text { by membrane } \\
\text { filtration, modified } \\
\text { mTEC agar method, } \\
\text { CFU per } 100 \mathrm{~mL} \\
\end{array}$ \\
\hline \multicolumn{5}{|c|}{02481194 Bayou Bernard (Canal Road) near Landon, MS [HN-6] } \\
\hline 9/19/2005 & 1520 & $56(38-77)$ & & \\
\hline $9 / 26 / 2005$ & 1350 & $145(100-205)$ & & \\
\hline $10 / 3 / 2005$ & 1345 & $71(50-95)$ & & \\
\hline $10 / 11 / 2005$ & 1505 & $87(64-115)$ & & \\
\hline $10 / 17 / 2005$ & 1400 & $24(15-35)$ & & \\
\hline \multicolumn{5}{|c|}{02481252 Turkey Creek at Creosote Road near Gulfport, MS [HN-7] } \\
\hline 9/20/2005 & 1340 & $186(125-269)$ & & \\
\hline $9 / 27 / 2005$ & 1300 & $181(104-286)$ & 340 & 70 \\
\hline $10 / 4 / 2005$ & 1245 & $63(25-127)$ & & \\
\hline $10 / 12 / 2005$ & 0905 & $41(17-95)$ & & \\
\hline $10 / 18 / 2005$ & 1430 & $10(1-55)$ & $40 \mathrm{E}$ & $58 \mathrm{E}$ \\
\hline \multicolumn{5}{|c|}{02481130 Biloxi River near Lyman, MS [HN-8] } \\
\hline 9/20/2005 & 1415 & $28(18-41)$ & & \\
\hline $9 / 27 / 2005$ & 1005 & $63(45-85)$ & & \\
\hline $10 / 12 / 2005$ & 1425 & $12(6-21)$ & & \\
\hline $10 / 18 / 2005$ & 1000 & $17(9-27)$ & & \\
\hline \multicolumn{5}{|c|}{02480606 Howard Creek at Cedar Lake, MS [HN-9] } \\
\hline 9/20/2005 & 1455 & $101(74-136)$ & & \\
\hline $9 / 27 / 2005$ & 0940 & $261(171-398)$ & & \\
\hline $10 / 4 / 2005$ & 1450 & $378(262-526)$ & 510 & 137 \\
\hline $10 / 12 / 2005$ & 1355 & $249(173-350)$ & & \\
\hline $10 / 18 / 2005$ & 0940 & $437(337-556)$ & & \\
\hline \multicolumn{5}{|c|}{02480590 Tchoutacabouffa River near D'Iberville, MS [HN-10] } \\
\hline 9/20/2005 & 1505 & $1(0-4)$ & & \\
\hline \multicolumn{5}{|c|}{02480595 Cypress Creek near D'Iberville, MS [HN-11] } \\
\hline 9/20/2005 & 1510 & $4(2-10)$ & & \\
\hline \multicolumn{5}{|c|}{301827089173955 Mississippi Sound at Henderson Point, MS [HNB-1] } \\
\hline 9/19/2005 & 1710 & $<10(0-37)$ & & \\
\hline $9 / 26 / 2005$ & 1640 & $10(1-55)$ & & \\
\hline $10 / 3 / 2005$ & 1550 & $20(3-56)$ & 90 & $10 \mathrm{E}$ \\
\hline $10 / 12 / 2005$ & 1110 & $<10(0-37)$ & & \\
\hline $10 / 18 / 2005$ & 1200 & $<10(0-37)$ & $8 \mathrm{E}$ & $<3$ \\
\hline
\end{tabular}


Table 4. Continued--Results of bacteriological analyses for water samples collected in the aftermath of Hurricane Katrina, coastal Mississippi Counties, September 19 - October 18, 2005

\begin{tabular}{|c|c|c|c|c|}
\hline Sample date & Sample time & $\begin{array}{c}\text { Enterococci } \\
\text { densities analyzed } \\
\text { by Enterolert most } \\
\text { probable number } \\
\text { method, } \\
\text { MPN per } 100 \mathrm{~mL} \\
\end{array}$ & $\begin{array}{c}\text { Enterococci } \\
\text { densities analyzed } \\
\text { by membrane } \\
\text { filtration, mEl agar } \\
\text { method, } \\
\text { CFU per } 100 \mathrm{~mL}\end{array}$ & $\begin{array}{c}\text { Escherichia Coli } \\
\text { densities analyzed } \\
\text { by membrane } \\
\text { filtration, modified } \\
\text { mTEC agar method, } \\
\text { CFU per } 100 \mathrm{~mL} \\
\end{array}$ \\
\hline \multicolumn{5}{|c|}{301843089145255 Mississippi Sound at Pass Christian Harbour at Pass Christian, MS [HNB-2] } \\
\hline 9/19/2005 & 1640 & $85(39-156)$ & & \\
\hline $9 / 26 / 2005$ & 1605 & $10(1-55)$ & $9 \mathrm{E}$ & $7 \mathrm{E}$ \\
\hline $10 / 3 / 2005$ & 1520 & $120(60-203)$ & & \\
\hline 10/12/2005 & 1050 & $<10(0-37)$ & & \\
\hline $10 / 18 / 2005$ & 1130 & $41(17-95)$ & & \\
\hline \multicolumn{5}{|c|}{302044089083655 Mississippi Sound at Long Beach, MS [HNB-3] } \\
\hline 9/19/2005 & 1620 & $10(1-55)$ & & \\
\hline $9 / 26 / 2005$ & 1430 & $323(211-472)$ & & \\
\hline 10/4/2005 & 0840 & $262(166-397)$ & & \\
\hline 10/12/2005 & 1020 & 109 (56-195) & & \\
\hline 10/18/2005 & 1100 & $31(7-89)$ & & \\
\hline \multicolumn{5}{|c|}{302147089051055 Mississipi Sound at Gulfport Harbor at Gulfport, MS [HNB-4] } \\
\hline 9/20/2005 & 1525 & $132(71-220)$ & & \\
\hline 9/27/2005 & 1410 & $40(11-89)$ & & \\
\hline $10 / 4 / 2005$ & 1305 & $337(227-480)$ & & \\
\hline 10/12/2005 & 1005 & $1918(1367-2645)$ & & \\
\hline 10/18/2005 & 1210 & $<10(0-37)$ & & \\
\hline \multicolumn{5}{|c|}{302237089025855 Mississippi Sound at Maywood Road at Gulfport, MS [HNB-5] } \\
\hline 9/20/2005 & 1535 & $645(460-873)$ & & \\
\hline $10 / 4 / 2005$ & 1315 & $73(29-139)$ & & \\
\hline 10/12/2005 & 1630 & $<10(0-37)$ & & \\
\hline 10/18/2005 & 1200 & $757(569-985)$ & 2500 & 2400 \\
\hline \multicolumn{5}{|c|}{302328088575655 Mississippi Sound at Broadwater Marina at Biloxi, MS [HNB-6] } \\
\hline 9/20/2005 & 1600 & $448(302-634)$ & & \\
\hline $9 / 27 / 2005$ & 1425 & $63(29-137)$ & & \\
\hline $10 / 4 / 2005$ & 1335 & $20(3-71)$ & & \\
\hline $10 / 12 / 2005$ & 1615 & $<10(0-37)$ & & \\
\hline 10/18/2005 & 1145 & $109(56-195)$ & & \\
\hline \multicolumn{5}{|c|}{302318088512600 Biloxi Bay at Point Cadet Harbor at Biloxi, MS [HNB-7] } \\
\hline 9/20/2005 & 1620 & $10(1-55)$ & & \\
\hline 9/27/2005 & 1445 & $10(0-37)$ & & \\
\hline $10 / 4 / 2005$ & 1350 & $<10(0-37)$ & & \\
\hline 10/12/2005 & 1300 & $<10(0-37)$ & & \\
\hline 10/18/2005 & 1125 & $<10(0-37)$ & & \\
\hline
\end{tabular}


Table 4. Continued--Results of bacteriological analyses for water samples collected in the aftermath of Hurricane Katrina, coastal Mississippi Counties, September 19 - October 18, 2005

\begin{tabular}{|c|c|c|c|c|}
\hline Sample date & Sample time & $\begin{array}{c}\text { Enterococci } \\
\text { densities analyzed } \\
\text { by Enterolert most } \\
\text { probable number } \\
\text { method, } \\
\text { MPN per } 100 \mathrm{~mL} \\
\end{array}$ & $\begin{array}{c}\text { Enterococci } \\
\text { densities analyzed } \\
\text { by membrane } \\
\text { filtration, mEl agar } \\
\text { method, } \\
\text { CFU per } 100 \mathrm{~mL}\end{array}$ & $\begin{array}{c}\text { Escherichia Coli } \\
\text { densities analyzed } \\
\text { by membrane } \\
\text { filtration, modified } \\
\text { mTEC agar method, } \\
\text { CFU per } 100 \mathrm{~mL} \\
\end{array}$ \\
\hline \multicolumn{5}{|c|}{02481299 Old Fort Bayou at Ocean Springs, MS [JK-1] } \\
\hline 9/20/2005 & 1230 & $10(1-55)$ & & \\
\hline \multicolumn{5}{|c|}{02481292 Perigal Creek near D'Iberville, MS [JK-2] } \\
\hline 9/20/2005 & 1315 & $27(17-39)$ & & \\
\hline 9/27/2005 & 0905 & $317(264-378)$ & & \\
\hline $10 / 4 / 2005$ & 0930 & $326(270-388)$ & & \\
\hline $10 / 12 / 2005$ & 0630 & $31(21-44)$ & & \\
\hline $10 / 17 / 2005$ & 1855 & $4(2-10)$ & & \\
\hline \multicolumn{5}{|c|}{0248129250 South Branch Perigal Creek near Ocean Springs, MS [JK-3] } \\
\hline 9/20/2005 & 1250 & $4(2-10)$ & & \\
\hline \multicolumn{5}{|c|}{02480289 Davis Bayou at Ocean Springs, MS [JK-4] } \\
\hline 9/20/2005 & 1155 & $122(68-214)$ & & \\
\hline 9/27/2005 & 0835 & $644(446-886)$ & & \\
\hline 10/4/2005 & 0850 & $86(45-169)$ & & \\
\hline $10 / 12 / 2005$ & 1005 & $146(82-246)$ & & \\
\hline $10 / 17 / 2005$ & 1810 & $135(78-234)$ & & \\
\hline \multicolumn{5}{|c|}{02480288 Simmons Bayou at Ocean Springs, MS [JK-5] } \\
\hline 9/20/2005 & 1125 & $41(17-95)$ & & \\
\hline $9 / 27 / 2005$ & 0815 & $74(36-149)$ & & \\
\hline $10 / 4 / 2005$ & 0830 & $98(47-184)$ & & \\
\hline 10/12/2005 & 0905 & $20(3-71)$ & & \\
\hline $10 / 17 / 2005$ & 1755 & $41(17-95)$ & & \\
\hline \multicolumn{5}{|c|}{02480284 Mary Walker Bayou at Gautier, MS [JK-6] } \\
\hline 9/20/2005 & 1105 & $72(53-95)$ & & \\
\hline $9 / 26 / 2005$ & 1600 & $461(293-688)$ & & \\
\hline $10 / 3 / 2005$ & 1545 & $74(32-144)$ & 280 & 155 \\
\hline $10 / 11 / 2005$ & 1810 & $10(1-55)$ & & \\
\hline $10 / 17 / 2005$ & 1720 & $10(0-37)$ & & \\
\hline \multicolumn{5}{|c|}{02480286 Bayou St. Pierre at Gautier, MS [JK-7] } \\
\hline 10/11/2005 & 1740 & $10(0-37)$ & & \\
\hline \multicolumn{5}{|c|}{02480285 West Pascagoula at Highway 90 at Gautier, MS [JK-7A] } \\
\hline 9/19/2005 & 1715 & $10(1-55)$ & & \\
\hline 9/26/2005 & 1545 & $108(52-186)$ & & \\
\hline $10 / 3 / 2005$ & 1530 & $20(3-59)$ & & \\
\hline 10/17/2005 & 1705 & $<10(0-37)$ & & \\
\hline
\end{tabular}


Table 4. Continued--Results of bacteriological analyses for water samples collected in the aftermath of Hurricane Katrina, coastal Mississippi Counties, September 19 - October 18, 2005

\begin{tabular}{|c|c|c|c|c|}
\hline Sample date & Sample time & $\begin{array}{c}\text { Enterococci } \\
\text { densities analyzed } \\
\text { by Enterolert most } \\
\text { probable number } \\
\text { method, } \\
\text { MPN per } 100 \mathrm{~mL} \\
\end{array}$ & $\begin{array}{c}\text { Enterococci } \\
\text { densities analyzed } \\
\text { by membrane } \\
\text { filtration, mEl agar } \\
\text { method, } \\
\text { CFU per } 100 \mathrm{~mL} \\
\end{array}$ & $\begin{array}{l}\text { Escherichia Coli } \\
\text { densities analyzed } \\
\text { by membrane } \\
\text { filtration, modified } \\
\text { mTEC agar method, } \\
\text { CFU per } 100 \mathrm{~mL} \\
\end{array}$ \\
\hline \multicolumn{5}{|c|}{02480205 Black Creek near Helena, MS [JK-8] } \\
\hline 9/20/2005 & 0915 & $49(36-65)$ & & \\
\hline 9/26/2005 & 1415 & $91(66-123)$ & 280 & 440 \\
\hline 10/3/2005 & 1425 & $6(3-14)$ & & \\
\hline $10 / 11 / 2005$ & 1525 & $15(8-25)$ & & \\
\hline $10 / 17 / 2005$ & 1555 & $1(0-4)$ & & \\
\hline \multicolumn{5}{|c|}{0247109250 Bayou Chico at Pascagoula, MS [JK-9] } \\
\hline 9/20/2005 & 1005 & $288(183-427)$ & & \\
\hline 9/26/2005 & 1500 & 1785 (1238-2505) & & \\
\hline $10 / 3 / 2005$ & 1455 & $160(95-251)$ & & \\
\hline $10 / 11 / 2005$ & 1640 & 24196 (16304-47161) & & \\
\hline $10 / 17 / 2005$ & 1625 & $<10(0-37)$ & & \\
\hline \multicolumn{5}{|c|}{0247109160 West Prong Bayou Cassotte at Louise Road at Pascagoula, MS [JK-10] } \\
\hline 9/20/2005 & 0950 & $85(39-156)$ & & \\
\hline 9/26/2005 & 1450 & $3076(1953-4712)$ & & \\
\hline $10 / 3 / 2005$ & 1445 & $41(17-95)$ & & \\
\hline $10 / 11 / 2005$ & 1625 & $10(1-55)$ & & \\
\hline $10 / 17 / 2005$ & 1620 & $<10(0-37)$ & & \\
\hline \multicolumn{5}{|c|}{0247109150 Bayou Cassotte at Orchard Avenue at Pascagoula, MS [JK-11] } \\
\hline 9/20/2005 & 0945 & $81(37-153)$ & & \\
\hline 9/26/2005 & 1440 & $121(65-211)$ & & \\
\hline $10 / 3 / 2005$ & 1400 & $41(17-95)$ & $21 \mathrm{E}$ & 160 \\
\hline 10/11/2005 & 1615 & $10(1-55)$ & & \\
\hline $10 / 17 / 2005$ & 1615 & $<10(0-37)$ & & \\
\hline \multicolumn{5}{|c|}{302421088495255 Biloxi Bay at Jackson Avenue at Ocean Springs, MS [JKB-1] } \\
\hline 9/20/2005 & 1210 & $20(3-71)$ & & \\
\hline 9/27/2005 & 0845 & $161(93-268)$ & $202 E$ & 130 \\
\hline $10 / 4 / 2005$ & 0910 & $52(23-119)$ & & \\
\hline $10 / 12 / 2005$ & 1025 & $<10(0-37)$ & $12 \mathrm{E}$ & $<3$ \\
\hline $10 / 17 / 2005$ & 1835 & $41(17-95)$ & & \\
\hline \multicolumn{5}{|c|}{302036088320955 Mississippi Sound at Country Club Drive at Pascagoula, MS [JKB-2] } \\
\hline 9/20/2005 & 1025 & $<10(0-37)$ & & \\
\hline 9/26/2005 & 1515 & $1137(833-1517)$ & & \\
\hline $10 / 3 / 2005$ & 1510 & $20(3-71)$ & & \\
\hline $10 / 11 / 2005$ & 1705 & $<10(0-37)$ & $<3$ & $<3$ \\
\hline 10/17/2005 & 1635 & $10(1-55)$ & & \\
\hline
\end{tabular}


Table 4. Continued--Results of bacteriological analyses for water samples collected in the aftermath of Hurricane Katrina, coastal Mississippi Counties, September 19 - October 18, 2005

\begin{tabular}{|c|c|c|c|c|}
\hline Sample date & Sample time & $\begin{array}{c}\text { Enterococci } \\
\text { densities analyzed } \\
\text { by Enterolert most } \\
\text { probable number } \\
\text { method, } \\
\text { MPN per } 100 \mathrm{~mL}\end{array}$ & $\begin{array}{c}\text { Enterococci } \\
\text { densities analyzed } \\
\text { by membrane } \\
\text { filtration, mEl agar } \\
\text { method, } \\
\text { CFU per } 100 \mathrm{~mL}\end{array}$ & $\begin{array}{c}\text { Escherichia Coli } \\
\text { densities analyzed } \\
\text { by membrane } \\
\text { filtration, modified } \\
\text { mTEC agar method, } \\
\text { CFU per } 100 \mathrm{~mL} \\
\end{array}$ \\
\hline \multicolumn{5}{|c|}{302031088313855 Mississippi Sound near Chico Bayou at Pascagoula, MS [JKB-3] } \\
\hline 9/20/2005 & 1035 & $<10(0-37)$ & & \\
\hline 9/26/2005 & 1520 & $31(7-89)$ & & \\
\hline $10 / 3 / 2005$ & 1515 & $<10(0-37)$ & & \\
\hline $10 / 11 / 2005$ & 1655 & $<10(0-37)$ & & \\
\hline $10 / 17 / 2005$ & 1645 & $41(17-95)$ & & \\
\hline
\end{tabular}


Table 5. U.S. Environmental Protection Agency criteria for Enterococci and Escherichia coli in recreational waters

[CFU, colony-forming units]

\begin{tabular}{lccc}
\hline \multirow{2}{*}{ Fecal indicator bacteria } & \multicolumn{2}{c}{$\begin{array}{c}\text { Single-sample density (CFU per 100 milliliters; not to be } \\
\text { exceeded in more than 10 percent of samples taken over a } \\
\text { 30-day period)* }\end{array}$} \\
\cline { 2 - 4 } & $\begin{array}{c}\text { 30-dignated beach } \\
\text { areas }\end{array}$ & $\begin{array}{c}\text { Moderate full-body } \\
\text { contact }\end{array}$ & $\begin{array}{c}\text { Infrequent full- } \\
\text { body contact }\end{array}$ \\
\hline $\begin{array}{c}\text { Freshwater } \\
\text { Enterococci }\end{array}$ & 61 & 78 & 151 \\
Escherichia coli & 235 & 298 & 575 \\
Marine water & & & 501 \\
\hline Enterococci & 104 & 158 & \\
\hline
\end{tabular}

* U.S. Environmental Protection Agency (2004a) 
Table 6. Results of bacteriological quality-assurance samples collected in the aftermath of Hurricane Katrina, coastal Mississippi Counties, September 19 - October 18, 2005.

[MPN, most probable number; numbers in parentheses are environmental sample results for comparison; >, greater than; QC, quality control; <, less than]

\begin{tabular}{|c|c|c|c|c|c|}
\hline Sample/Site & Type & $\begin{array}{c}\text { Sample } \\
\text { date }\end{array}$ & $\begin{array}{c}\text { Sample } \\
\text { time }\end{array}$ & $\begin{array}{c}\text { Time } \\
\text { processed }\end{array}$ & $\begin{array}{c}\text { Enterococci concentrations } \\
\text { analyzed by Enterolert most } \\
\text { probable number method, } \\
\text { MPN per } 100 \mathrm{~mL}\end{array}$ \\
\hline \multicolumn{6}{|l|}{ REPLICATES } \\
\hline 02481671 & replicate & $10 / 4 / 2005$ & 1615 & 1805 & $41(52)$ \\
\hline 02481526 & replicate & $10 / 12 / 2005$ & 1210 & 1650 & $>2420(326)$ \\
\hline 02481194 & replicate & 10/17/2005 & 1400 & 1745 & $14(24)$ \\
\hline 302044089083655 & replicate & $10 / 4 / 2005$ & 840 & 1430 & $148(262)$ \\
\hline 302044089083655 & replicate & 10/12/2005 & 1020 & 1448 & 95 (109) \\
\hline 302044089083655 & replicate & $10 / 18 / 2005$ & 1100 & 1615 & $41(31)$ \\
\hline 302147089051055 & replicate & 10/4/2005 & 1305 & 1810 & 262 (337) \\
\hline 02480284 & replicate & 9/26/2005 & 1600 & 2040 & 201 (461) \\
\hline 02480285 & replicate & 9/19/2005 & 1715 & 2125 & $20(10)$ \\
\hline 302421088495255 & replicate & 9/20/2005 & 1210 & 1635 & $10(20)$ \\
\hline 302031088313855 & replicate & $9 / 26 / 2005$ & 1520 & 2025 & $20(31)$ \\
\hline \multicolumn{6}{|c|}{ BLANKS } \\
\hline Blank & QC & 9/19/2005 & & 2125 & $<1$ \\
\hline Blank & QC & 9/20/2005 & & 1415 & $<1$ \\
\hline Blank & QC & $9 / 26 / 2005$ & & 2015 & $<1$ \\
\hline Blank & QC & 9/27/2005 & & 1335 & $<1$ \\
\hline Blank & QC & $10 / 4 / 2005$ & & 1315 & $<1$ \\
\hline Blank & QC & $10 / 4 / 2005$ & & 1630 & $<1$ \\
\hline Blank & QC & 10/11/2005 & & 2155 & $<1$ \\
\hline Blank & QC & 10/12/2005 & & 1620 & $<1$ \\
\hline Blank & QC & 10/18/2005 & & 1820 & $<1$ \\
\hline Blank & QC & $10 / 18 / 2005$ & & 1215 & $<1$ \\
\hline Blank & $\mathrm{QC}$ & $10 / 17 / 2005$ & & 2010 & $<1$ \\
\hline Field blank & QC & 10/18/2005 & 1030 & 1620 & 8 \\
\hline \multicolumn{6}{|c|}{ POSITIVE/NEGATIVE CONTROLS } \\
\hline Positive control & QC & 9/20/2005 & & 1710 & $>2400$ \\
\hline Positive control & QC & $9 / 27 / 2005$ & & 1335 & $>2420$ \\
\hline Positive control & QC & $10 / 4 / 2005$ & & 1630 & $>2420$ \\
\hline Positive control & $\mathrm{QC}$ & $10 / 12 / 2005$ & & 1620 & $>2420$ \\
\hline Positive control & QC & $10 / 17 / 2005$ & & 2015 & $>2420$ \\
\hline Negative control & QC & 9/20/2005 & & 1710 & $<1$ \\
\hline Negative control & QC & 9/27/2005 & & 1335 & $<1$ \\
\hline Negative control & $\mathrm{QC}$ & $10 / 4 / 2005$ & & 1630 & $<1$ \\
\hline Negative control & QC & 10/12/2005 & & 1620 & $<1$ \\
\hline Negative control & QC & 10/17/2005 & & 2015 & $<1$ \\
\hline
\end{tabular}


Table 7. Constituents and compounds included for analysis for samples collected in the aftermath of Hurricane Katrina, southeastern Mississippi, September 19-30, 2005

[cfs, cubic feet per second; NTU, Nephlometric Turbidity Units; mg/L, milligrams per liter; uS/cm, microsiemens per centimeter; C, Celsius; N, nitrogen; P, phosphorus;

$\mathrm{ug} / \mathrm{L}$, micrograms per liter; compounds in bold were not detected in any sample]

\begin{tabular}{lcc}
\hline Analyte & $\begin{array}{c}\text { Reporting } \\
\text { level }\end{array}$ & Units \\
\hline PHYSICAL PROPERTIES & & \\
Discharge & 0 & $\mathrm{cfs}$ \\
Turbidity & 0 & $\mathrm{NTU}$ \\
Dissolved oxygen & 0.1 & $\mathrm{mg} / \mathrm{L}$ \\
pH & 0.1 & $\mathrm{pH}$ \\
Specific conductance & 2.6 & $\mathrm{uS} / \mathrm{cm}$ \\
Temperature & 0.1 & $\mathrm{C}$ \\
& & \\
NITROGEN, PHOSPHORUS, CARBON, AND OXYGEN DEMAND & & \\
Ammonia plus organic nitrogen as N, filtered & 0.1 & $\mathrm{mg} / \mathrm{L}$ \\
Ammonia plus organic nitrogen as N, unfiltered & 0.1 & $\mathrm{mg} / \mathrm{L}$ \\
Ammonia as N, filtered & 0.04 & $\mathrm{mg} / \mathrm{L}$ \\
Nitrite plus nitrate as N, filtered & 0.06 & $\mathrm{mg} / \mathrm{L}$ \\
Nitrite as N, filtered & 0.008 & $\mathrm{mg} / \mathrm{L}$ \\
Orthophosphate as P, filtered & 0.018 & $\mathrm{mg} / \mathrm{L}$ \\
Phosphorus, filtered & 0.004 & $\mathrm{mg} / \mathrm{L}$ \\
Phosphorus, unfiltered & 0.004 & $\mathrm{mg} / \mathrm{L}$ \\
Organic carbon, unfiltered & 0.4 & $\mathrm{mg} / \mathrm{L}$ \\
Biochemical oxygen demand, unfiltered & 1 & $\mathrm{mg} / \mathrm{L}$ \\
Chemical oxygen demand, high level, unfiltered & 10 & $\mathrm{mg} / \mathrm{L}$
\end{tabular}

MAJOR IONS, METALS, AND RELATED CHEMISTRY (all constituents are from filtered sample)

$\begin{array}{lcc}\text { Calcium } & 0.02 & \mathrm{mg} / \mathrm{L} \\ \text { Magnesium } & 0.008 & \mathrm{mg} / \mathrm{L} \\ \text { Potassium } & 0.16 & \mathrm{mg} / \mathrm{L} \\ \text { Sodium } & 0.2 & \mathrm{mg} / \mathrm{L} \\ \text { Alkalinity } & 1 & \mathrm{mg} / \mathrm{L} \\ \text { Bicarbonate } & 1 & \mathrm{mg} / \mathrm{L} \\ \text { Chloride } & 0.2 & \mathrm{mg} / \mathrm{L} \\ \text { Fluoride } & 0.1 & \mathrm{mg} / \mathrm{L} \\ \text { Silica } & 0.04 & \mathrm{mg} / \mathrm{L} \\ \text { Sulfate } & 0.18 & \mathrm{mg} / \mathrm{L} \\ \text { Residue on evaporation, 180 degrees Celsius } & 10 & \mathrm{mg} / \mathrm{L} \\ \text { Aluminum } & 1.6 & \mathrm{ug} / \mathrm{L} \\ \text { Antimony } & 0.2 & \mathrm{ug} / \mathrm{L} \\ \text { Arsenic } & 0.12 & \mathrm{ug} / \mathrm{L} \\ \text { Barium } & 0.2 & \mathrm{ug} / \mathrm{L} \\ \text { Beryllium } & 0.06 & \mathrm{ug} / \mathrm{L} \\ \text { Boron } & 8 & \mathrm{ug} / \mathrm{L} \\ \text { Cadmium } & 0.04 & \mathrm{ug} / \mathrm{L} \\ \text { Chromium } & 0.04 & \mathrm{ug} / \mathrm{L} \\ \text { Cobalt } & 0.04 & \mathrm{ug} / \mathrm{L} \\ \text { Copper } & 0.4 & \mathrm{ug} / \mathrm{L} \\ \text { Iron } & 6 & \mathrm{ug} / \mathrm{L} \\ \text { Lead } & 0.08 & \mathrm{ug} / \mathrm{L} \\ \text { Lithium } & 0.6 & \mathrm{ug} / \mathrm{L} \\ \text { Manganese } & 0.6 & \mathrm{ug} / \mathrm{L} \\ \text { Molybdenum } & 0.4 & \mathrm{ug} / \mathrm{L} \\ \text { Nickel } & 0.06 & \mathrm{ug} / \mathrm{L}\end{array}$


Table 7. Continued--Constituents and compounds included for analysis for samples collected in the aftermath of Hurricane Katrina, southeastern Mississippi, September 19-30, 2005

\begin{tabular}{lcc}
\hline Analyte & $\begin{array}{c}\text { Reporting } \\
\text { level }\end{array}$ & Units \\
\hline Selenium & 0.08 & $\mathrm{ug} / \mathrm{L}$ \\
Silver & 0.2 & $\mathrm{ug} / \mathrm{L}$ \\
Strontium & 0.4 & $\mathrm{ug} / \mathrm{L}$ \\
Thallium & 0.04 & $\mathrm{ug} / \mathrm{L}$ \\
Uranium, natural & 0.04 & $\mathrm{ug} / \mathrm{L}$ \\
Vanadium & 0.1 & $\mathrm{ug} / \mathrm{L}$ \\
Zinc & 0.6 & $\mathrm{ug} / \mathrm{L}$
\end{tabular}

MODERN USE PESTICIDES AND DEGRADATES (all constituents are from filtered sample)

1-Naphthol

2-Chloro-2,6-diethylacetanilide

2-Ethyl-6-methylaniline

3,4-Dichloroaniline

3,5-Dichloroaniline

4-Chloro-2-methylphenol

Acetochlor

Alachlor

2,6-Diethylaniline

Atrazine

Azinphos-methyl

Azinphos-methyl-oxon

Benfluralin

Carbaryl

Carbofuran

Chlorpyrifos

Chlorpyrofos, oxygen analog

cis-Permethrin

cis-Propiconazole

Cyanazine

Cyfluthrin

Cypermethrin

Dacthal

2-Chloro-4-isopropylamino-6-amino-s-triazine $\{\mathrm{CIAT}\}$

Diazinon

Dichlorvos

Dicrotophos

Dieldrin

Dimethoate

Disulfoton

Disulfoton sulfone

alpha-Endosulfan

Endosulfan sulfate

EPTC

Ethion

Ethion monoxon

Ethoprophos

Fenamiphos

Fenamiphos sulfone

Fenamiphos sulfoxide

Desulfinylfipronil amide

Fipronil sulfide

Fipronil sulfone

Desulfinylfipronil

$\begin{array}{cc}0.0882 & \mathrm{ug} / \mathrm{L} \\ 0.005 & \mathrm{ug} / \mathrm{L} \\ 0.0045 & \mathrm{ug} / \mathrm{L} \\ 0.0045 & \mathrm{ug} / \mathrm{L} \\ 0.0043 & \mathrm{ug} / \mathrm{L} \\ 0.0056 & \mathrm{ug} / \mathrm{L} \\ 0.006 & \mathrm{ug} / \mathrm{L} \\ 0.005 & \mathrm{ug} / \mathrm{L} \\ 0.006 & \mathrm{ug} / \mathrm{L} \\ 0.007 & \mathrm{ug} / \mathrm{L} \\ 0.05 & \mathrm{ug} / \mathrm{L} \\ 0.07 & \mathrm{ug} / \mathrm{L} \\ 0.01 & \mathrm{ug} / \mathrm{L} \\ 0.041 & \mathrm{ug} / \mathrm{L} \\ 0.02 & \mathrm{ug} / \mathrm{L} \\ 0.005 & \mathrm{ug} / \mathrm{L} \\ 0.0562 & \mathrm{ug} / \mathrm{L} \\ 0.006 & \mathrm{ug} / \mathrm{L} \\ 0.008 & \mathrm{ug} / \mathrm{L} \\ 0.018 & \mathrm{ug} / \mathrm{L} \\ 0.0267 & \mathrm{ug} / \mathrm{L} \\ 0.0086 & \mathrm{ug} / \mathrm{L} \\ 0.003 & \mathrm{ug} / \mathrm{L} \\ 0.006 & \mathrm{ug} / \mathrm{L} \\ 0.005 & \mathrm{ug} / \mathrm{L} \\ 0.0118 & \mathrm{ug} / \mathrm{L} \\ 0.0843 & \mathrm{ug} / \mathrm{L} \\ 0.009 & \mathrm{ug} / \mathrm{L} \\ 0.0061 & \mathrm{ug} / \mathrm{L} \\ 0.021 & \mathrm{ug} / \mathrm{L} \\ 0.0059 & \mathrm{ug} / \mathrm{L} \\ 0.0047 & \mathrm{ug} / \mathrm{L} \\ 0.0138 & \mathrm{ug} / \mathrm{L} \\ 0.004 & \mathrm{ug} / \mathrm{L} \\ 0.004 & \mathrm{ug} / \mathrm{L} \\ 0.002 & \mathrm{ug} / \mathrm{L} \\ 0.005 & \mathrm{ug} / \mathrm{L} \\ 0.029 & \mathrm{ug} / \mathrm{L} \\ 0.0491 & \mathrm{ug} / \mathrm{L} \\ 0.0387 & \mathrm{ug} / \mathrm{L} \\ 0.029 & \mathrm{ug} / \mathrm{L} \\ 0.013 & \mathrm{ug} / \mathrm{L} \\ & \end{array}$


Table 7. Continued--Constituents and compounds included for analysis for samples collected in the aftermath of Hurricane Katrina, southeastern Mississippi, September 19-30, 2005

\begin{tabular}{|c|c|c|}
\hline Analyte & $\begin{array}{c}\text { Reporting } \\
\text { level }\end{array}$ & Units \\
\hline Fipronil & 0.016 & ug/L \\
\hline Fonofos & 0.003 & $\mathrm{ug} / \mathrm{L}$ \\
\hline Hexazinone & 0.0129 & $\mathrm{ug} / \mathrm{L}$ \\
\hline Iprodione & 0.538 & $\mathrm{ug} / \mathrm{L}$ \\
\hline Isofenphos & 0.0034 & $\mathrm{ug} / \mathrm{L}$ \\
\hline lambda-Cyhalothrin & 0.0089 & $\mathrm{ug} / \mathrm{L}$ \\
\hline Malaoxon & 0.0298 & $\mathrm{ug} / \mathrm{L}$ \\
\hline Malathion & 0.027 & $\mathrm{ug} / \mathrm{L}$ \\
\hline Metalaxyl & 0.0051 & $\mathrm{ug} / \mathrm{L}$ \\
\hline Methidathion & 0.0058 & $\mathrm{ug} / \mathrm{L}$ \\
\hline Parathion-methyl & 0.015 & $\mathrm{ug} / \mathrm{L}$ \\
\hline Metolachlor & 0.006 & $\mathrm{ug} / \mathrm{L}$ \\
\hline Metribuzin & 0.006 & $\mathrm{ug} / \mathrm{L}$ \\
\hline Molinate & 0.003 & $\mathrm{ug} / \mathrm{L}$ \\
\hline Myclobutanil & 0.008 & $\mathrm{ug} / \mathrm{L}$ \\
\hline Oxyfluorfen & 0.0073 & $\mathrm{ug} / \mathrm{L}$ \\
\hline Paraoxon-methyl & 0.0299 & $\mathrm{ug} / \mathrm{L}$ \\
\hline Pendimethalin & 0.022 & $\mathrm{ug} / \mathrm{L}$ \\
\hline Phorate & 0.011 & $\mathrm{ug} / \mathrm{L}$ \\
\hline Phorate oxygen analog & 0.1048 & $\mathrm{ug} / \mathrm{L}$ \\
\hline Phosmet & 0.0079 & $\mathrm{ug} / \mathrm{L}$ \\
\hline Phosmet oxon & 0.0511 & $\mathrm{ug} / \mathrm{L}$ \\
\hline Prometon & 0.01 & $\mathrm{ug} / \mathrm{L}$ \\
\hline Prometryn & 0.0054 & $\mathrm{ug} / \mathrm{L}$ \\
\hline Propyzamide & 0.004 & $\mathrm{ug} / \mathrm{L}$ \\
\hline Propanil & 0.011 & $\mathrm{ug} / \mathrm{L}$ \\
\hline Propargite & 0.023 & $\mathrm{ug} / \mathrm{L}$ \\
\hline Simazine & 0.005 & $\mathrm{ug} / \mathrm{L}$ \\
\hline Tebuconazole & 0.0136 & $\mathrm{ug} / \mathrm{L}$ \\
\hline Tebuthiuron & 0.016 & $\mathrm{ug} / \mathrm{L}$ \\
\hline Tefluthrin & 0.0077 & $\mathrm{ug} / \mathrm{L}$ \\
\hline Terbufos & 0.017 & $\mathrm{ug} / \mathrm{L}$ \\
\hline Terbufos oxygen analog sulfone & 0.0676 & $\mathrm{ug} / \mathrm{L}$ \\
\hline Terbuthylazine & 0.0102 & $\mathrm{ug} / \mathrm{L}$ \\
\hline Thiobencarb & 0.01 & $\mathrm{ug} / \mathrm{L}$ \\
\hline trans-Propiconazole & 0.0133 & $\mathrm{ug} / \mathrm{L}$ \\
\hline Tribufos & 0.0044 & $\mathrm{ug} / \mathrm{L}$ \\
\hline Trifluralin & 0.009 & $\mathrm{ug} / \mathrm{L}$ \\
\hline \multicolumn{3}{|c|}{ POLAR PESTICIDES AND DEGRADATES (all constituents are from filtered sample) } \\
\hline 2,4-D & 0.038 & $\mathrm{ug} / \mathrm{L}$ \\
\hline 2,4-D methyl ester & 0.016 & $\mathrm{ug} / \mathrm{L}$ \\
\hline 2,4-DB & 0.02 & $\mathrm{ug} / \mathrm{L}$ \\
\hline 2-Hydroxy-4-isopropylamino-6-ethylamino-s-triazine $\{$ OIET\} & 0.032 & $\mathrm{ug} / \mathrm{L}$ \\
\hline 3(4-Chlorophenyl)-1-methyl urea & 0.036 & $\mathrm{ug} / \mathrm{L}$ \\
\hline Acifluorfen & 0.028 & $\mathrm{ug} / \mathrm{L}$ \\
\hline Aldicarb & 0.04 & $\mathrm{ug} / \mathrm{L}$ \\
\hline Aldicarb sulfone & 0.018 & $\mathrm{ug} / \mathrm{L}$ \\
\hline Aldicarb sulfoxide & 0.022 & $\mathrm{ug} / \mathrm{L}$ \\
\hline Chloramben, methyl ester & 0.024 & $\mathrm{ug} / \mathrm{L}$ \\
\hline Atrazine & 0.008 & $\mathrm{ug} / \mathrm{L}$ \\
\hline Bendiocarb & 0.02 & $\mathrm{ug} / \mathrm{L}$ \\
\hline Benomyl & 0.022 & $\mathrm{ug} / \mathrm{L}$ \\
\hline
\end{tabular}


Table 7. Continued--Constituents and compounds included for analysis for samples collected in the aftermath of Hurricane Katrina, southeastern Mississippi, September 19-30, 2005

\begin{tabular}{|c|c|c|}
\hline Analyte & $\begin{array}{l}\text { Reporting } \\
\text { level }\end{array}$ & Units \\
\hline Bensulfuron-methyl & 0.018 & $\mathrm{ug} / \mathrm{L}$ \\
\hline Bentazon & 0.012 & ug/L \\
\hline Bromacil & 0.018 & ug/L \\
\hline Bromoxynil & 0.028 & ug/L \\
\hline Caffeine & 0.018 & ug/L \\
\hline Carbaryl & 0.018 & ug/L \\
\hline Carbofuran & 0.016 & $\mathrm{ug} / \mathrm{L}$ \\
\hline 3-Hydroxycarbofuran & 0.008 & ug/L \\
\hline Chlorimuron-ethyl & 0.032 & ug/L \\
\hline Clopyralid & 0.024 & $\mathrm{ug} / \mathrm{L}$ \\
\hline Cycloate & 0.014 & $\mathrm{ug} / \mathrm{L}$ \\
\hline Dacthal monoacid & 0.028 & $\mathrm{ug} / \mathrm{L}$ \\
\hline 2-Chloro-4-isopropylamino-6-amino-s-triazine $\{\mathrm{CIAT}\}$ & 0.028 & ug/L \\
\hline Chlordiamino-s-triazine & 0.04 & ug/L \\
\hline 2-Chloro-6-ethylamino-4-amino-s-triazine $\{C E A T\}$ & 0.08 & $\mathrm{ug} / \mathrm{L}$ \\
\hline Dicamba & 0.036 & $\mathrm{ug} / \mathrm{L}$ \\
\hline Dichlorprop & 0.028 & ug/L \\
\hline Dinoseb & 0.038 & ug/L \\
\hline Diphenamid & 0.01 & $\mathrm{ug} / \mathrm{L}$ \\
\hline Diuron & 0.015 & ug/L \\
\hline Fenuron & 0.019 & $\mathrm{ug} / \mathrm{L}$ \\
\hline Flumetsulam & 0.04 & $\mathrm{ug} / \mathrm{L}$ \\
\hline Fluometuron & 0.016 & ug/L \\
\hline Imazaquin & 0.036 & $\mathrm{ug} / \mathrm{L}$ \\
\hline Imazethapyr & 0.038 & ug/L \\
\hline Imidacloprid & 0.02 & ug/L \\
\hline Linuron & 0.014 & ug/L \\
\hline MCPA & 0.03 & $\mathrm{ug} / \mathrm{L}$ \\
\hline MCPB & 0.01 & $\mathrm{ug} / \mathrm{L}$ \\
\hline Metalaxyl & 0.012 & ug/L \\
\hline Methiocarb & 0.01 & $\mathrm{ug} / \mathrm{L}$ \\
\hline Methomyl & 0.02 & ug/L \\
\hline Metsulfuron methyl & 0.025 & $\mathrm{ug} / \mathrm{L}$ \\
\hline Neburon & 0.012 & $\mathrm{ug} / \mathrm{L}$ \\
\hline Nicosulfuron & 0.04 & $\mathrm{ug} / \mathrm{L}$ \\
\hline Norflurazon & 0.02 & $\mathrm{ug} / \mathrm{L}$ \\
\hline Oryzalin & 0.012 & $\mathrm{ug} / \mathrm{L}$ \\
\hline Oxamyl & 0.03 & ug/L \\
\hline Picloram & 0.032 & $\mathrm{ug} / \mathrm{L}$ \\
\hline Propham & 0.03 & $\mathrm{ug} / \mathrm{L}$ \\
\hline Propiconazole & 0.01 & $\mathrm{ug} / \mathrm{L}$ \\
\hline Propoxur & 0.008 & ug/L \\
\hline Siduron & 0.02 & $\mathrm{ug} / \mathrm{L}$ \\
\hline Sulfometuron-methyl & 0.038 & ug/L \\
\hline Tebuthiuron & 0.026 & ug/L \\
\hline Terbacil & 0.016 & ug/L \\
\hline Triclopyr & 0.026 & ug/L \\
\hline \multicolumn{3}{|c|}{$\begin{array}{l}\text { WASTE WATER ORGANIC COMPOUNDS AND DEGRADATES } \\
\text { (all constituents are from filtered sample) }\end{array}$} \\
\hline Cotinine & 1 & ug/L \\
\hline 5-Methyl-1H-benzotriazole & 2 & $\mathrm{ug} / \mathrm{L}$ \\
\hline Anthraquinone & 0.5 & $\mathrm{ug} / \mathrm{L}$ \\
\hline
\end{tabular}


Table 7. Continued--Constituents and compounds included for analysis for samples collected in the aftermath of Hurricane Katrina, southeastern Mississippi, September 19-30, 2005

\begin{tabular}{|c|c|c|}
\hline Analyte & $\begin{array}{c}\text { Reporting } \\
\text { level }\end{array}$ & Units \\
\hline Acetophenone & 0.5 & $\mathrm{ug} / \mathrm{L}$ \\
\hline Acetyl hexamethyl tetrahydronaphthalene (AHTN) & 0.5 & ug/L \\
\hline Anthracene & 0.5 & ug/L \\
\hline 1,4-Dichlorobenzene & 0.5 & ug/L \\
\hline Benzo[a]pyrene & 0.5 & ug/L \\
\hline Benzophenone & 0.5 & ug/L \\
\hline Bromacil & 0.5 & ug/L \\
\hline Bromoform & 0.5 & ug/L \\
\hline 3-tert-Butyl-4-hydroxy anisole (BHA) & 5 & ug/L \\
\hline Caffeine & 0.5 & ug/L \\
\hline Camphor & 0.5 & ug/L \\
\hline Carbaryl & 1 & $\mathrm{ug} / \mathrm{L}$ \\
\hline Carbazole & 0.5 & $\mathrm{ug} / \mathrm{L}$ \\
\hline Chlorpyrifos & 0.5 & $\mathrm{ug} / \mathrm{L}$ \\
\hline Cholesterol & 2 & $\mathrm{ug} / \mathrm{L}$ \\
\hline 3-beta-Coprostanol & 2 & $\mathrm{ug} / \mathrm{L}$ \\
\hline Isopropylbenzene & 0.5 & $\mathrm{ug} / \mathrm{L}$ \\
\hline N,N-diethyl-meta-toluamide (DEET) & 0.5 & $\mathrm{ug} / \mathrm{L}$ \\
\hline Diazinon & 0.5 & $\mathrm{ug} / \mathrm{L}$ \\
\hline Dichlorvos & 1 & $\mathrm{ug} / \mathrm{L}$ \\
\hline Bisphenol A & 1 & $\mathrm{ug} / \mathrm{L}$ \\
\hline Triethyl citrate (ethyl citrate) & 0.5 & $\mathrm{ug} / \mathrm{L}$ \\
\hline Tetrachloroethylene & 0.5 & $\mathrm{ug} / \mathrm{L}$ \\
\hline Fluoranthene & 0.5 & $\mathrm{ug} / \mathrm{L}$ \\
\hline Hexahydrohexamethylcyclopentabenzopyran (HHCB) & 0.5 & $\mathrm{ug} / \mathrm{L}$ \\
\hline Indole & 0.5 & $\mathrm{ug} / \mathrm{L}$ \\
\hline Isoborneol & 0.5 & $\mathrm{ug} / \mathrm{L}$ \\
\hline Isophorone & 0.5 & $\mathrm{ug} / \mathrm{L}$ \\
\hline Isoquinoline & 0.5 & $\mathrm{ug} / \mathrm{L}$ \\
\hline d-Limonene & 0.5 & $\mathrm{ug} / \mathrm{L}$ \\
\hline Menthol & 0.5 & $\mathrm{ug} / \mathrm{L}$ \\
\hline Metalaxyl & 0.5 & $\mathrm{ug} / \mathrm{L}$ \\
\hline Metolachlor & 0.5 & $\mathrm{ug} / \mathrm{L}$ \\
\hline Naphthalene & 0.5 & $\mathrm{ug} / \mathrm{L}$ \\
\hline 1-Methylnaphthalene & 0.5 & $\mathrm{ug} / \mathrm{L}$ \\
\hline 2,6-Dimethylnaphthalene & 0.5 & $\mathrm{ug} / \mathrm{L}$ \\
\hline 2-Methylnaphthalene & 0.5 & $\mathrm{ug} / \mathrm{L}$ \\
\hline 4-Nonylphenol diethoxylates & 5 & $\mathrm{ug} / \mathrm{L}$ \\
\hline 4-Octylphenol diethoxylates & 1 & $\mathrm{ug} / \mathrm{L}$ \\
\hline 4-Octylphenol monoethoxylates & 1 & $\mathrm{ug} / \mathrm{L}$ \\
\hline p-Cresol & 1 & $\mathrm{ug} / \mathrm{L}$ \\
\hline 4-Cumylphenol & 1 & $\mathrm{ug} / \mathrm{L}$ \\
\hline para-Nonylphenol (total) & 5 & $\mathrm{ug} / \mathrm{L}$ \\
\hline 4-n-Octylphenol & 1 & $\mathrm{ug} / \mathrm{L}$ \\
\hline 4-tert-Octylphenol & 1 & $\mathrm{ug} / \mathrm{L}$ \\
\hline Phenanthrene & 0.5 & $\mathrm{ug} / \mathrm{L}$ \\
\hline Phenol & 0.5 & $\mathrm{ug} / \mathrm{L}$ \\
\hline Pentachlorophenol & 2 & $\mathrm{ug} / \mathrm{L}$ \\
\hline Tributyl phosphate & 0.5 & $\mathrm{ug} / \mathrm{L}$ \\
\hline Triphenyl phosphate & 0.5 & $\mathrm{ug} / \mathrm{L}$ \\
\hline Tris(2-butoxyethyl)phosphate & 0.5 & $\mathrm{ug} / \mathrm{L}$ \\
\hline Tris(2-chloroethyl)phosphate & 0.5 & $\mathrm{ug} / \mathrm{L}$ \\
\hline Prometon & 0.5 & $\mathrm{ug} / \mathrm{L}$ \\
\hline
\end{tabular}


Table 7. Continued--Constituents and compounds included for analysis for samples collected in the aftermath of Hurricane Katrina, southeastern Mississippi, September 19-30, 2005

\begin{tabular}{|c|c|c|}
\hline Analyte & $\begin{array}{c}\text { Reporting } \\
\text { level }\end{array}$ & Units \\
\hline Pyrene & 0.5 & $\mathrm{ug} / \mathrm{L}$ \\
\hline Methyl salicylate & 0.5 & ug/L \\
\hline 3-Methyl-1(H)-indole (Skatole) & 1 & ug/L \\
\hline beta-Sitosterol & 2 & ug/L \\
\hline beta-Stigmastanol & 2 & ug/L \\
\hline Triclosan & 1 & $\mathrm{ug} / \mathrm{L}$ \\
\hline Tris(dichlorisopropyl)phosphate & 0.5 & ug/L \\
\hline \multicolumn{3}{|c|}{$\begin{array}{l}\text { VOLATILE ORGANIC COMPOUNDS AND DEGRADATES } \\
\text { (all constituents are from unfiltered sample) }\end{array}$} \\
\hline 1,2,3,4-Tetramethylbenzene & 0.14 & ug/L \\
\hline 1,2,3,5-Tetramethylbenzene & 0.14 & $\mathrm{ug} / \mathrm{L}$ \\
\hline 2-Butanone & 2 & ug/L \\
\hline trans-1,4-Dichloro-2-butene & 0.7 & ug/L \\
\hline 2-Hexanone & 0.4 & $\mathrm{ug} / \mathrm{L}$ \\
\hline 4-Methyl-2-pentanone & 0.37 & ug/L \\
\hline Acetone & 6 & ug/L \\
\hline Acrylonitrile & 0.8 & $\mathrm{ug} / \mathrm{L}$ \\
\hline Benzene & 0.021 & $\mathrm{ug} / \mathrm{L}$ \\
\hline 1,2,3-Trichlorobenzene & 0.18 & ug/L \\
\hline 1,2,3-Trimethylbenzene & 0.06 & ug/L \\
\hline 1,2,4-Trichlorobenzene & 0.12 & ug/L \\
\hline Bromobenzene & 0.028 & ug/L \\
\hline Chlorobenzene & 0.028 & $\mathrm{ug} / \mathrm{L}$ \\
\hline Ethylbenzene & 0.03 & ug/L \\
\hline 1,3-Dichlorobenzene & 0.03 & ug/L \\
\hline Butylbenzene & 0.12 & ug/L \\
\hline n-Propylbenzene & 0.042 & ug/L \\
\hline 1,2-Dichlorobenzene & 0.048 & ug/L \\
\hline 1,4-Dichlorobenzene & 0.034 & $\mathrm{ug} / \mathrm{L}$ \\
\hline sec-Butylbenzene & 0.06 & ug/L \\
\hline tert-Butylbenzene & 0.06 & ug/L \\
\hline Bromoethene & 0.1 & $\mathrm{ug} / \mathrm{L}$ \\
\hline Bromoform & 0.1 & ug/L \\
\hline Hexachlorobutadiene & 0.14 & ug/L \\
\hline Carbon disulfide & 0.038 & $\mathrm{ug} / \mathrm{L}$ \\
\hline Tetrachloromethane & 0.06 & ug/L \\
\hline Chloroform & 0.024 & ug/L \\
\hline Isopropylbenzene & 0.038 & ug/L \\
\hline 1,1,1,2-Tetrachloroethane & 0.03 & ug/L \\
\hline 1,1,1-Trichloroethane & 0.032 & ug/L \\
\hline 1,1,2-Trichlorotrifluoroethane & 0.038 & $\mathrm{ug} / \mathrm{L}$ \\
\hline 1,2-Dibromoethane & 0.036 & $\mathrm{ug} / \mathrm{L}$ \\
\hline 1,2-Dichloroethane & 0.13 & ug/L \\
\hline Hexachloroethane & 0.14 & $\mathrm{ug} / \mathrm{L}$ \\
\hline 1,1,2,2-Tetrachloroethane & 0.08 & ug/L \\
\hline Chloroethane & 0.12 & ug/L \\
\hline Diethyl ether & 0.08 & $\mathrm{ug} / \mathrm{L}$ \\
\hline Ethyl tert-butyl ether & 0.03 & ug/L \\
\hline cis-1,2-Dichloroethylene & 0.024 & ug/L \\
\hline Tetrachloroethylene & 0.03 & $\mathrm{ug} / \mathrm{L}$ \\
\hline trans-1,2-Dichloroethylene & 0.032 & $\mathrm{ug} / \mathrm{L}$ \\
\hline Trichloroethylene & 0.038 & ug/L \\
\hline
\end{tabular}


Table 7. Continued--Constituents and compounds included for analysis for samples collected in the aftermath of Hurricane Katrina, southeastern Mississippi, September 19-30, 2005

\begin{tabular}{|c|c|c|}
\hline Analyte & $\begin{array}{c}\text { Reporting } \\
\text { level }\end{array}$ & Units \\
\hline 1,1-Dichloroethane & 0.035 & ug/L \\
\hline Tetrahydrofuran & 1 & ug/L \\
\hline Diisopropyl ether & 0.1 & ug/L \\
\hline m- and p-Xylene & 0.06 & $\mathrm{ug} / \mathrm{L}$ \\
\hline 1,3,5-Trimethylbenzene & 0.044 & $\mathrm{ug} / \mathrm{L}$ \\
\hline Ethyl methacrylate & 0.18 & $\mathrm{ug} / \mathrm{L}$ \\
\hline Methyl methacrylate & 0.2 & $\mathrm{ug} / \mathrm{L}$ \\
\hline Methyl acrylonitrile & 0.4 & $\mathrm{ug} / \mathrm{L}$ \\
\hline Bromochloromethane & 0.12 & ug/L \\
\hline Bromodichloromethane & 0.028 & ug/L \\
\hline Dibromochloromethane & 0.1 & ug/L \\
\hline Dichlorodifluoromethane & 0.18 & $\mathrm{ug} / \mathrm{L}$ \\
\hline Trichlorofluoromethane & 0.08 & ug/L \\
\hline Methyl acrylate & 1 & $\mathrm{ug} / \mathrm{L}$ \\
\hline Bromomethane & 0.26 & $\mathrm{ug} / \mathrm{L}$ \\
\hline Chloromethane & 0.17 & $\mathrm{ug} / \mathrm{L}$ \\
\hline Methyl iodide & 0.5 & ug/L \\
\hline tert-Butyl methyl ether & 0.1 & $\mathrm{ug} / \mathrm{L}$ \\
\hline Dibromomethane & 0.05 & $\mathrm{ug} / \mathrm{L}$ \\
\hline Dichloromethane & 0.06 & $\mathrm{ug} / \mathrm{L}$ \\
\hline Naphthalene & 0.52 & $\mathrm{ug} / \mathrm{L}$ \\
\hline o-Xylene & 0.038 & $\mathrm{ug} / \mathrm{L}$ \\
\hline 4-Isopropyl-1-methylbenzene & 0.08 & ug/L \\
\hline 1,2,3-Trichloropropane & 0.18 & $\mathrm{ug} / \mathrm{L}$ \\
\hline 1,3-Dichloropropane & 0.06 & ug/L \\
\hline 2,2-Dichloropropane & 0.05 & ug/L \\
\hline 1,2-Dibromo-3-chloropropane & 0.51 & $\mathrm{ug} / \mathrm{L}$ \\
\hline 1,1-Dichloropropene & 0.026 & $\mathrm{ug} / \mathrm{L}$ \\
\hline 3-Chloropropene & 0.5 & $\mathrm{ug} / \mathrm{L}$ \\
\hline cis-1,3-Dichloropropene & 0.05 & $\mathrm{ug} / \mathrm{L}$ \\
\hline trans-1,3-Dichloropropene & 0.09 & $\mathrm{ug} / \mathrm{L}$ \\
\hline 1,2-Dichloropropane & 0.029 & $\mathrm{ug} / \mathrm{L}$ \\
\hline 1,2,4-Trimethylbenzene & 0.056 & $\mathrm{ug} / \mathrm{L}$ \\
\hline Styrene & 0.042 & $\mathrm{ug} / \mathrm{L}$ \\
\hline tert-Pentyl methyl ether & 0.04 & ug/L \\
\hline Toluene & 0.02 & $\mathrm{ug} / \mathrm{L}$ \\
\hline 2-Chlorotoluene & 0.04 & $\mathrm{ug} / \mathrm{L}$ \\
\hline o-Ethyl toluene & 0.06 & $\mathrm{ug} / \mathrm{L}$ \\
\hline 4-Chlorotoluene & 0.05 & $\mathrm{ug} / \mathrm{L}$ \\
\hline Vinyl chloride & 0.08 & $\mathrm{ug} / \mathrm{L}$ \\
\hline 1,1,2-Trichloroethane & 0.04 & $\mathrm{ug} / \mathrm{L}$ \\
\hline 1,1-Dichloroethylene & 0.024 & ug/L \\
\hline
\end{tabular}


Table 8. Constituents and compounds detected in samples collected in the aftermath of Hurricane Katrina, southeastern Mississippi September 19-30, 2005.

[MS, Mississippi; inst, instantaneous; E, estimated (typically below laboratory reporting level); water-quality criteria values with a slash (/) are acute and chronic values, respectively, unless otherwise stated; CMC, criteria maximum concentration (related to acute toxicity); CCC, criteria continuous concentration (related to chronic toxicity); residue upon evaporation (parameter code 70300) is equivalent to total dissolved solids; recom, recommended]

\begin{tabular}{|c|c|c|c|}
\hline $\begin{array}{l}\text { Parameter } \\
\text { code }\end{array}$ & Parameter name & Result & $\begin{array}{l}\text { Water-quality criteria } \\
\text { or recommendation }\end{array}$ \\
\hline \multicolumn{4}{|c|}{02475000 Leaf River near McClain, MS, Sample Date: 9/27/05, Sample Time: 1000} \\
\hline \multicolumn{4}{|c|}{ Physical properties } \\
\hline 00061 & Discharge, instantaneous, cubic feet per second & 1420 & \\
\hline 63676 & Turbidity, water, unfiltered, Nephlometric Turbidity Ratio-Units & 19 & \\
\hline 00300 & Dissolved oxygen, water, unfiltered, milligrams per liter & 6.5 & 5 (4 inst) $^{\mathrm{a}}$ \\
\hline 00400 & $\mathrm{pH}$, water, unfiltered, field, standard units & 6.5 & $6-9^{\mathrm{a}}$ \\
\hline 00095 & Specific conductance, water, unfiltered, microsiemens per centimeter at 25 degrees Celsius & 157 & $1000^{\mathrm{a}}$ \\
\hline 00010 & Temperature, water, degrees Celsius & 27.8 & $32.2^{\mathrm{a}}$ \\
\hline \multicolumn{4}{|c|}{ Nitrogen, Phosphorus, Carbon, and Oxygen Demand } \\
\hline 00623 & Ammonia plus organic nitrogen, water, filtered, milligrams per liter as nitrogen & 0.41 & \\
\hline 00625 & Ammonia plus organic nitrogen, water, unfiltered, milligrams per liter as nitrogen & 0.48 & \\
\hline 00608 & Ammonia, water, filtered, milligrams per liter as nitrogen & E0.03 & $1.5-2^{b}$ \\
\hline 00631 & Nitrite plus nitrate, water, filtered, milligrams per liter as nitrogen & 0.34 & \\
\hline 00613 & Nitrite, water, filtered, milligrams per liter as nitrogen & 0.008 & \\
\hline 00671 & Orthophosphate, water, filtered, milligrams per liter as phosphorus & 0.04 & \\
\hline 00666 & Phosphorus, water, filtered, milligrams per liter & 0.064 & \\
\hline 00665 & Phosphorus, water, unfiltered, milligrams per liter & 0.137 & $0.1^{\mathrm{c}}$ \\
\hline 00680 & Organic carbon, water, unfiltered, milligrams per liter & 8.1 & \\
\hline 00310 & Biochemical oxygen demand, water, unfiltered, 5 days at 20 degrees Celsius, milligrams per liter & 2 & \\
\hline 00340 & Chemical oxygen demand, high level, water, unfiltered, milligrams per liter & 20 & \\
\hline \multicolumn{4}{|c|}{ Major ions, metals, and related chemistry } \\
\hline 00915 & Calcium, water, filtered, milligrams per liter & 5.36 & \\
\hline 00925 & Magnesium, water, filtered, milligrams per liter & 1.57 & \\
\hline 00935 & Potassium, water, filtered, milligrams per liter & 3.33 & \\
\hline 00930 & Sodium, water, filtered, milligrams per liter & 22.4 & \\
\hline 39086 & Alkalinity, water, filtered, incremental titration, field, milligrams per liter as calcium carbonate & 25 & \\
\hline 00453 & Bicarbonate, water, filtered, incremental titration, field, milligrams per liter & 30 & \\
\hline 00940 & Chloride, water, filtered, milligrams per liter & 15.7 & $860(\mathrm{CMC}) / 230(\mathrm{CCC})^{d}$ \\
\hline 00955 & Silica, water, filtered, milligrams per liter & 12.3 & \\
\hline 00945 & Sulfate, water, filtered, milligrams per liter & 14.5 & \\
\hline 70300 & Residue on evaporation, dried at 180 degrees Celsius, water, filtered, milligrams per liter & 112 & $1,500^{\mathrm{a}}$ \\
\hline 01106 & Aluminum, water, filtered, micrograms per liter & 21 & $750(\mathrm{CMC}) / 87(\mathrm{CCC})^{\mathrm{d}}$ \\
\hline 01000 & Arsenic, water, filtered, micrograms per liter & 0.9 & $340 / 150^{\mathrm{a}}$ \\
\hline 01005 & Barium, water, filtered, micrograms per liter & 31 & $50,000(\text { recom })^{c}$ \\
\hline 01020 & Boron, water, filtered, micrograms per liter & 20 & \\
\hline 01030 & Chromium, water, filtered, micrograms per liter & 0.18 & $16 / 11^{\mathrm{a}}$ \\
\hline 01035 & Cobalt, water, filtered, micrograms per liter & 0.229 & \\
\hline 01040 & Copper, water, filtered, micrograms per liter & 1.3 & $7 / 5^{\mathrm{a}}$ \\
\hline 01046 & Iron, water, filtered, micrograms per liter & 149 & $1000^{d}$ \\
\hline 01049 & Lead, water, filtered, micrograms per liter & 0.09 & $30 / 1.18^{\mathrm{a}}$ \\
\hline 01130 & Lithium, water, filtered, micrograms per liter & 1.2 & \\
\hline 01056 & Manganese, water, filtered, micrograms per liter & 59.7 & \\
\hline 01060 & Molybdenum, water, filtered, micrograms per liter & 0.4 & \\
\hline 01065 & Nickel, water, filtered, micrograms per liter & 0.97 & $260 / 29^{\mathrm{a}}$ \\
\hline 01080 & Strontium, water, filtered, micrograms per liter & 36.3 & \\
\hline 22703 & Uranium (natural), water, filtered, micrograms per liter & E0.04 & \\
\hline 01085 & Vanadium, water, filtered, micrograms per liter & 1.1 & \\
\hline 01090 & Zinc, water, filtered, micrograms per liter & 1.2 & $65 / 65^{\mathrm{a}}$ \\
\hline \multicolumn{4}{|c|}{ Organic chemicals } \\
\hline 50305 & Caffeine, water, filtered, recoverable, micrograms per liter & 0.028 & \\
\hline 62083 & Diethoxynonylphenol, water, filtered, recoverable, micrograms per liter & E3 & \\
\hline 32106 & Trichloromethane, water, unfiltered, recoverable, micrograms per liter & E0.02 & \\
\hline \multicolumn{4}{|c|}{02478500 Chickasawhay River at Leakesville, MS, Sample Date: 9/27/05, Sample Time: 1330} \\
\hline \multicolumn{4}{|c|}{ Physical properties } \\
\hline 00061 & Discharge, instantaneous, cubic feet per second & 1750 & \\
\hline 63676 & Turbidity, water, unfiltered, Nephlometric Turbidity Ratio-Units & 73 & \\
\hline 00300 & Dissolved oxygen, water, unfiltered, milligrams per liter & 6.8 & 5 (4 inst) $^{\mathrm{a}}$ \\
\hline 00400 & $\mathrm{pH}$, water, unfiltered, field, standard units & 6.7 & $6-9^{a}$ \\
\hline 00095 & Specific conductance, water, unfiltered, microsiemens per centimeter at 25 degrees Celsius & 88 & $1000^{\mathrm{a}}$ \\
\hline
\end{tabular}


Table 8. Continued--Constituents and compounds detected in samples collected in the aftermath of Hurricane Katrina, southeastern Mississippi September 19-30, 2005.

\begin{tabular}{|c|c|c|c|}
\hline $\begin{array}{l}\text { Parameter } \\
\text { code }\end{array}$ & Parameter name & Result & $\begin{array}{l}\text { Water-quality criteria } \\
\text { or recommendation }\end{array}$ \\
\hline 00010 & Temperature, water, degrees Celsius & 28.3 & $32.2^{\mathrm{a}}$ \\
\hline \multicolumn{4}{|c|}{ Nitrogen, Phosphorus, Carbon, and Oxygen Demand } \\
\hline 00623 & Ammonia plus organic nitrogen, water, filtered, milligrams per liter as nitrogen & 0.22 & \\
\hline 00625 & Ammonia plus organic nitrogen, water, unfiltered, milligrams per liter as nitrogen & 0.47 & \\
\hline 00631 & Nitrite plus nitrate, water, filtered, milligrams per liter as nitrogen & 0.22 & \\
\hline 00613 & Nitrite, water, filtered, milligrams per liter as nitrogen & E0.004 & \\
\hline 00666 & Phosphorus, water, filtered, milligrams per liter & 0.006 & \\
\hline 00665 & Phosphorus, water, unfiltered, milligrams per liter & 0.052 & $0.1^{\mathrm{c}}$ \\
\hline 00680 & Organic carbon, water, unfiltered, milligrams per liter & 6.9 & \\
\hline 00340 & Chemical oxygen demand, high level, water, unfiltered, milligrams per liter & 20 & \\
\hline \multicolumn{4}{|c|}{ Major ions, metals, and related chemistry } \\
\hline 00915 & Calcium, water, filtered, milligrams per liter & 8.06 & \\
\hline 00925 & Magnesium, water, filtered, milligrams per liter & 1.5 & \\
\hline 00935 & Potassium, water, filtered, milligrams per liter & 1.73 & \\
\hline 00930 & Sodium, water, filtered, milligrams per liter & 5.51 & \\
\hline 39086 & Alkalinity, water, filtered, incremental titration, field, milligrams per liter as calcium carbonate & 21 & \\
\hline 00453 & Bicarbonate, water, filtered, incremental titration, field, milligrams per liter & E25 & \\
\hline 00940 & Chloride, water, filtered, milligrams per liter & 6.3 & $860(\mathrm{CMC}) / 230(\mathrm{CCC})^{d}$ \\
\hline 00950 & Fluoride, water, filtered, milligrams per liter & E0.1 & \\
\hline 00955 & Silica, water, filtered, milligrams per liter & 10.8 & \\
\hline 00945 & Sulfate, water, filtered, milligrams per liter & 4.2 & \\
\hline 70300 & Residue on evaporation, dried at 180 degrees Celsius, water, filtered, milligrams per liter & 72 & $1,500^{\mathrm{a}}$ \\
\hline 01106 & Aluminum, water, filtered, micrograms per liter & 9 & $750(\mathrm{CMC}) / 87(\mathrm{CCC})^{\mathrm{d}}$ \\
\hline 01000 & Arsenic, water, filtered, micrograms per liter & 0.5 & $340 / 150^{a}$ \\
\hline 01005 & Barium, water, filtered, micrograms per liter & 25 & $50,000(\text { recom })^{c}$ \\
\hline 01020 & Boron, water, filtered, micrograms per liter & 19 & \\
\hline 01030 & Chromium, water, filtered, micrograms per liter & 0.09 & $16 / 11^{\mathrm{a}}$ \\
\hline 01035 & Cobalt, water, filtered, micrograms per liter & 0.138 & \\
\hline 01040 & Copper, water, filtered, micrograms per liter & 0.7 & $7 / 5^{a}$ \\
\hline 01046 & Iron, water, filtered, micrograms per liter & 135 & $1000^{d}$ \\
\hline 01049 & Lead, water, filtered, micrograms per liter & E0.06 & $30 / 1.18^{\mathrm{a}}$ \\
\hline 01130 & Lithium, water, filtered, micrograms per liter & 1.3 & \\
\hline 01056 & Manganese, water, filtered, micrograms per liter & 25.1 & \\
\hline 01065 & Nickel, water, filtered, micrograms per liter & 0.95 & $260 / 29^{\mathrm{a}}$ \\
\hline 01080 & Strontium, water, filtered, micrograms per liter & 66.6 & \\
\hline 22703 & Uranium (natural), water, filtered, micrograms per liter & E0.03 & \\
\hline 01085 & Vanadium, water, filtered, micrograms per liter & 0.3 & \\
\hline 01090 & Zinc, water, filtered, micrograms per liter & 0.9 & $65 / 65^{\mathrm{a}}$ \\
\hline \multicolumn{4}{|c|}{ Organic chemicals } \\
\hline 62083 & Diethoxynonylphenol, water, filtered, recoverable, micrograms per liter & E3 & \\
\hline 04025 & Hexazinone, water, filtered, recoverable, micrograms per liter & E0.011 & \\
\hline 50359 & Metalaxyl, water, filtered, recoverable, micrograms per liter & 0.71 & \\
\hline 04035 & Simazine, water, filtered, recoverable, micrograms per liter & 0.011 & \\
\hline \multicolumn{4}{|c|}{02479155 Cypress Creek near Janice, MS, Sample Date: 9/19/05, Sample Time: 1100} \\
\hline \multicolumn{4}{|c|}{ Physical properties } \\
\hline 00061 & Discharge, instantaneous, cubic feet per second & 69 & \\
\hline 00300 & Dissolved oxygen, water, unfiltered, milligrams per liter & 7.7 & 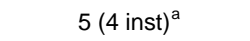 \\
\hline 00400 & $\mathrm{pH}$, water, unfiltered, field, standard units & 5 & $6-9^{a}$ \\
\hline 00095 & Specific conductance, water, unfiltered, microsiemens per centimeter at 25 degrees Celsius & 23 & $1000^{\mathrm{a}}$ \\
\hline 00010 & Temperature, water, degrees Celsius & 25.5 & $32.2^{\mathrm{a}}$ \\
\hline \multicolumn{4}{|c|}{ Nitrogen, Phosphorus, Carbon, and Oxygen Demand } \\
\hline 00623 & Ammonia plus organic nitrogen, water, filtered, milligrams per liter as nitrogen & 0.42 & \\
\hline 00625 & Ammonia plus organic nitrogen, water, unfiltered, milligrams per liter as nitrogen & 0.36 & \\
\hline 00608 & Ammonia, water, filtered, milligrams per liter as nitrogen & E0.02 & $1.5-2^{b}$ \\
\hline 00631 & Nitrite plus nitrate, water, filtered, milligrams per liter as nitrogen & E0.05 & \\
\hline 00666 & Phosphorus, water, filtered, milligrams per liter & 0.005 & \\
\hline 00665 & Phosphorus, water, unfiltered, milligrams per liter & 0.011 & $0.1^{\mathrm{c}}$ \\
\hline 00680 & Organic carbon, water, unfiltered, milligrams per liter & 10.1 & \\
\hline
\end{tabular}


Table 8. Continued--Constituents and compounds detected in samples collected in the aftermath of Hurricane Katrina, southeastern Mississippi September 19-30, 2005.

\begin{tabular}{|c|c|c|c|}
\hline $\begin{array}{l}\text { Parameter } \\
\text { code }\end{array}$ & Parameter name & Result & $\begin{array}{l}\text { Water-quality criteria } \\
\text { or recommendation }\end{array}$ \\
\hline \multicolumn{4}{|c|}{ Major ions, metals, and related chemistry } \\
\hline 00915 & Calcium, water, filtered, milligrams per liter & 0.76 & \\
\hline 00925 & Magnesium, water, filtered, milligrams per liter & 0.509 & \\
\hline 00935 & Potassium, water, filtered, milligrams per liter & 0.71 & \\
\hline 00930 & Sodium, water, filtered, milligrams per liter & 2.34 & \\
\hline 00940 & Chloride, water, filtered, milligrams per liter & 3.46 & $860(\mathrm{CMC}) / 230(\mathrm{CCC})^{\mathrm{d}}$ \\
\hline 00945 & Sulfate, water, filtered, milligrams per liter & 0.7 & \\
\hline 70300 & Residue on evaporation, dried at 180 degrees Celsius, water, filtered, milligrams per liter & 44 & $1,500^{\mathrm{a}}$ \\
\hline 01106 & Aluminum, water, filtered, micrograms per liter & 190 & $750(\mathrm{CMC}) / 87(\mathrm{CCC})^{d}$ \\
\hline 01000 & Arsenic, water, filtered, micrograms per liter & 0.6 & $340 / 150^{\mathrm{a}}$ \\
\hline 01005 & Barium, water, filtered, micrograms per liter & 30 & $50,000(\text { recom })^{\mathrm{c}}$ \\
\hline 01010 & Beryllium, water, filtered, micrograms per liter & 0.1 & $130 / 5.3^{c}$ \\
\hline 01020 & Boron, water, filtered, micrograms per liter & E4 & \\
\hline 01025 & Cadmium, water, filtered, micrograms per liter & E0.02 & $1.74 / 0.62^{\mathrm{a}}$ \\
\hline 01035 & Cobalt, water, filtered, micrograms per liter & 0.635 & \\
\hline 01040 & Copper, water, filtered, micrograms per liter & 0.8 & $7 / 5^{\mathrm{a}}$ \\
\hline 01046 & Iron, water, filtered, micrograms per liter & 496 & $1000^{d}$ \\
\hline 01049 & Lead, water, filtered, micrograms per liter & 0.32 & $30 / 1.18^{a}$ \\
\hline 01130 & Lithium, water, filtered, micrograms per liter & 0.9 & \\
\hline 01056 & Manganese, water, filtered, micrograms per liter & 47.5 & \\
\hline 01065 & Nickel, water, filtered, micrograms per liter & 1.03 & $260 / 29^{a}$ \\
\hline 01080 & Strontium, water, filtered, micrograms per liter & 8.66 & \\
\hline 22703 & Uranium (natural), water, filtered, micrograms per liter & 0.06 & \\
\hline 01085 & Vanadium, water, filtered, micrograms per liter & 0.5 & \\
\hline 01090 & Zinc, water, filtered, micrograms per liter & 2.9 & $65 / 65^{\mathrm{a}}$ \\
\hline \multicolumn{4}{|c|}{ Organic chemicals } \\
\hline 50305 & Caffeine, water, filtered, recoverable, micrograms per liter & E0.028 & \\
\hline 62070 & Camphor, water, filtered, recoverable, micrograms per liter & E0.1 & \\
\hline 62082 & DEET, water, filtered, recoverable, micrograms per liter & E0.1 & \\
\hline 77356 & 4-Isopropyltoluene, water, unfiltered, recoverable, micrograms per liter & E0.02 & \\
\hline \multicolumn{4}{|c|}{02479160 Black Creek near Wiggins, MS, Sample Date: 9/22/05, Sample Time: 1015} \\
\hline \multicolumn{4}{|c|}{ Physical properties } \\
\hline 00061 & Discharge, instantaneous, cubic feet per second & 384 & \\
\hline 00300 & Dissolved oxygen, water, unfiltered, milligrams per liter & 6.9 & 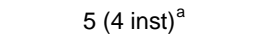 \\
\hline 00400 & $\mathrm{pH}$, water, unfiltered, field, standard units & 5.7 & $6-9^{\mathrm{a}}$ \\
\hline 00095 & Specific conductance, water, unfiltered, microsiemens per centimeter at 25 degrees Celsius & 34 & $1000^{\mathrm{a}}$ \\
\hline 00010 & Temperature, water, degrees Celsius & 26.8 & $32.2^{\mathrm{a}}$ \\
\hline \multicolumn{4}{|c|}{ Nitrogen, Phosphorus, Carbon, and Oxygen Demand } \\
\hline 00623 & Ammonia plus organic nitrogen, water, filtered, milligrams per liter as nitrogen & 0.35 & \\
\hline 00625 & Ammonia plus organic nitrogen, water, unfiltered, milligrams per liter as nitrogen & 0.32 & \\
\hline 00631 & Nitrite plus nitrate, water, filtered, milligrams per liter as nitrogen & E0.05 & \\
\hline 00666 & Phosphorus, water, filtered, milligrams per liter & 0.008 & \\
\hline 00665 & Phosphorus, water, unfiltered, milligrams per liter & 0.017 & $0.1^{\mathrm{c}}$ \\
\hline 00680 & Organic carbon, water, unfiltered, milligrams per liter & 7 & \\
\hline 00340 & Chemical oxygen demand, high level, water, unfiltered, milligrams per liter & 10 & \\
\hline \multicolumn{4}{|c|}{ Major ions, metals, and related chemistry } \\
\hline 00915 & Calcium, water, filtered, milligrams per liter & 1.48 & \\
\hline 00925 & Magnesium, water, filtered, milligrams per liter & 1.03 & \\
\hline 00935 & Potassium, water, filtered, milligrams per liter & 1.09 & \\
\hline 00930 & Sodium, water, filtered, milligrams per liter & 2.64 & \\
\hline 00940 & Chloride, water, filtered, milligrams per liter & 3.85 & $860(\mathrm{CMC}) / 230(\mathrm{CCC})^{\mathrm{d}}$ \\
\hline 00955 & Silica, water, filtered, milligrams per liter & 11 & \\
\hline 00945 & Sulfate, water, filtered, milligrams per liter & 1.7 & \\
\hline 70300 & Residue on evaporation, dried at 180 degrees Celsius, water, filtered, milligrams per liter & 44 & $1,500^{\mathrm{a}}$ \\
\hline 01106 & Aluminum, water, filtered, micrograms per liter & 70 & $750(\mathrm{CMC}) / 87(\mathrm{CCC})^{\mathrm{d}}$ \\
\hline 01000 & Arsenic, water, filtered, micrograms per liter & 0.8 & $340 / 150^{\mathrm{a}}$ \\
\hline 01005 & Barium, water, filtered, micrograms per liter & 29 & $50,000(\text { recom })^{\mathrm{c}}$ \\
\hline 01010 & Beryllium, water, filtered, micrograms per liter & E0.06 & $130 / 5.3^{\mathrm{c}}$ \\
\hline 01020 & Boron, water, filtered, micrograms per liter & 14 & \\
\hline 01030 & Chromium, water, filtered, micrograms per liter & 0.22 & $16 / 11^{\mathrm{a}}$ \\
\hline 01035 & Cobalt, water, filtered, micrograms per liter & 0.337 & \\
\hline
\end{tabular}


Table 8. Continued--Constituents and compounds detected in samples collected in the aftermath of Hurricane Katrina, southeastern Mississippi September 19-30, 2005.

\begin{tabular}{|c|c|c|c|}
\hline $\begin{array}{c}\text { Parameter } \\
\text { code }\end{array}$ & Parameter name & Result & $\begin{array}{l}\text { Water-quality criteria } \\
\text { or recommendation }\end{array}$ \\
\hline 01040 & Copper, water, filtered, micrograms per liter & 0.5 & $7 / 5^{\mathrm{a}}$ \\
\hline 01046 & Iron, water, filtered, micrograms per liter & 409 & $1000^{d}$ \\
\hline 01049 & Lead, water, filtered, micrograms per liter & 0.26 & $30 / 1.18^{a}$ \\
\hline 01130 & Lithium, water, filtered, micrograms per liter & 1 & \\
\hline 01056 & Manganese, water, filtered, micrograms per liter & 50 & \\
\hline 01065 & Nickel, water, filtered, micrograms per liter & 0.77 & $260 / 29^{a}$ \\
\hline 01145 & Selenium, water, filtered, micrograms per liter & E0.4 & $11.8 / 4.6^{\mathrm{a}}$ \\
\hline 01080 & Strontium, water, filtered, micrograms per liter & 11.9 & \\
\hline 22703 & Uranium (natural), water, filtered, micrograms per liter & E0.03 & \\
\hline 01085 & Vanadium, water, filtered, micrograms per liter & 0.4 & \\
\hline 01090 & Zinc, water, filtered, micrograms per liter & 1.4 & $65 / 65^{\mathrm{a}}$ \\
\hline \multicolumn{4}{|c|}{ Organic chemicals } \\
\hline 50305 & Caffeine, water, filtered, recoverable, micrograms per liter & E0.093 & \\
\hline 34010 & Toluene, water, unfiltered, recoverable, micrograms per liter & E0.08 & $6300 / 5000^{c}$ \\
\hline 00061 & Discharge, instantaneous, cubic feet per second & 404 & \\
\hline 63676 & Turbidity, water, unfiltered, Nephlometric Turbidity Ratio-Units & 5 & \\
\hline 00300 & Dissolved oxygen, water, unfiltered, milligrams per liter & 6.8 & $5(4 \text { inst })^{a}$ \\
\hline 00400 & $\mathrm{pH}$, water, unfiltered, field, standard units & 5.8 & $6-9^{a}$ \\
\hline 00095 & Specific conductance, water, unfiltered, microsiemens per centimeter at 25 degrees Celsius & 62 & $1000^{a}$ \\
\hline 00010 & Temperature, water, degrees Celsius & 27.6 & $32.2^{\mathrm{a}}$ \\
\hline \multicolumn{4}{|c|}{ Nitrogen, Phosphorus, Carbon, and Oxygen Demand } \\
\hline 00623 & Ammonia plus organic nitrogen, water, filtered, milligrams per liter as nitrogen & 0.38 & \\
\hline 00625 & Ammonia plus organic nitrogen, water, unfiltered, milligrams per liter as nitrogen & 0.35 & \\
\hline 00608 & Ammonia, water, filtered, milligrams per liter as nitrogen & E0.02 & $1.5-2^{b}$ \\
\hline 00631 & Nitrite plus nitrate, water, filtered, milligrams per liter as nitrogen & E0.06 & \\
\hline 00666 & Phosphorus, water, filtered, milligrams per liter & 0.005 & \\
\hline 00665 & Phosphorus, water, unfiltered, milligrams per liter & 0.014 & $0.1^{c}$ \\
\hline 00680 & Organic carbon, water, unfiltered, milligrams per liter & 8.3 & \\
\hline 00340 & Chemical oxygen demand, high level, water, unfiltered, milligrams per liter & 20 & \\
\hline 00925 & Magnesium, water, filtered, milligrams per liter & 2.27 & \\
\hline 00935 & Potassium, water, filtered, milligrams per liter & 1.25 & \\
\hline 00930 & Sodium, water, filtered, milligrams per liter & 3.99 & \\
\hline 39086 & Alkalinity, water, filtered, incremental titration, field, milligrams per liter as calcium carbonate & 5 & \\
\hline 00453 & Bicarbonate, water, filtered, incremental titration, field, milligrams per liter & 6 & \\
\hline 00940 & Chloride, water, filtered, milligrams per liter & 6.83 & $860(\mathrm{CMC}) / 230(\mathrm{CCC})^{d}$ \\
\hline 00950 & Fluoride, water, filtered, milligrams per liter & E0.1 & \\
\hline 00955 & Silica, water, filtered, milligrams per liter & 12 & \\
\hline 00945 & Sulfate, water, filtered, milligrams per liter & 7.1 & \\
\hline 70300 & Residue on evaporation, dried at 180 degrees Celsius, water, filtered, milligrams per liter & 65 & $1,500^{\mathrm{a}}$ \\
\hline 01106 & Aluminum, water, filtered, micrograms per liter & 47 & $750(\mathrm{CMC}) / 87(\mathrm{CCC})^{d}$ \\
\hline 01000 & Arsenic, water, filtered, micrograms per liter & 0.7 & $340 / 150^{\mathrm{a}}$ \\
\hline 01005 & Barium, water, filtered, micrograms per liter & 43 & $50,000(\text { recom })^{c}$ \\
\hline 01010 & Beryllium, water, filtered, micrograms per liter & E0.04 & $130 / 5.3^{c}$ \\
\hline 01020 & Boron, water, filtered, micrograms per liter & 55 & \\
\hline 01030 & Chromium, water, filtered, micrograms per liter & 0.2 & $16 / 11^{\mathrm{a}}$ \\
\hline 01035 & Cobalt, water, filtered, micrograms per liter & 0.382 & \\
\hline 01040 & Copper, water, filtered, micrograms per liter & 0.5 & $7 / 5^{a}$ \\
\hline 01046 & Iron, water, filtered, micrograms per liter & 261 & $1000^{d}$ \\
\hline 01049 & Lead, water, filtered, micrograms per liter & 0.12 & $30 / 1.18^{\mathrm{a}}$ \\
\hline 01130 & Lithium, water, filtered, micrograms per liter & 1.3 & \\
\hline 01056 & Manganese, water, filtered, micrograms per liter & 61.3 & \\
\hline 01065 & Nickel, water, filtered, micrograms per liter & 0.84 & $260 / 29^{a}$ \\
\hline 01080 & Strontium, water, filtered, micrograms per liter & 17.7 & \\
\hline 22703 & Uranium (natural), water, filtered, micrograms per liter & E0.03 & \\
\hline 01085 & Vanadium, water, filtered, micrograms per liter & 0.3 & \\
\hline 01090 & Zinc, water, filtered, micrograms per liter & 1.1 & $65 / 65^{\mathrm{a}}$ \\
\hline
\end{tabular}


Table 8. Continued--Constituents and compounds detected in samples collected in the aftermath of Hurricane Katrina, southeastern Mississippi September 19-30, 2005.

\begin{tabular}{|c|c|c|c|}
\hline $\begin{array}{l}\text { Parameter } \\
\text { code }\end{array}$ & Parameter name & Result & $\begin{array}{l}\text { Water-quality criteria } \\
\text { or recommendation }\end{array}$ \\
\hline \multicolumn{4}{|c|}{ Organic chemicals } \\
\hline 62085 & 4-Nonylphenol, water, filtered, recoverable, micrograms per liter & E2 & \\
\hline 50305 & Caffeine, water, filtered, recoverable, micrograms per liter & 0.08 & \\
\hline 62082 & DEET, water, filtered, recoverable, micrograms per liter & E0.1 & \\
\hline 62083 & Diethoxynonylphenol, water, filtered, recoverable, micrograms per liter & E10 & \\
\hline 34010 & Toluene, water, unfiltered, recoverable, micrograms per liter & E0.09 & $6300 / 5000^{c}$ \\
\hline \multicolumn{4}{|c|}{02479300 Red Creek at Vestry, MS, Sample Date: 9/20/05, Sample Time: 1500} \\
\hline \multicolumn{4}{|c|}{ Physical properties } \\
\hline 00061 & Discharge, instantaneous, cubic feet per second & 369 & \\
\hline 00300 & Dissolved oxygen, water, unfiltered, milligrams per liter & 6.4 & 5 (4 inst) $^{\mathrm{a}}$ \\
\hline 00400 & $\mathrm{pH}$, water, unfiltered, field, standard units & 6 & $6-9^{a}$ \\
\hline 00095 & Specific conductance, water, unfiltered, microsiemens per centimeter at 25 degrees Celsius & 41 & $1000^{\mathrm{a}}$ \\
\hline 00010 & Temperature, water, degrees Celsius & 28.3 & $32.2^{a}$ \\
\hline \multicolumn{4}{|c|}{ Nitrogen, Phosphorus, Carbon, and Oxygen Demand } \\
\hline 00623 & Ammonia plus organic nitrogen, water, filtered, milligrams per liter as nitrogen & 0.37 & \\
\hline 00625 & Ammonia plus organic nitrogen, water, unfiltered, milligrams per liter as nitrogen & 0.36 & \\
\hline 00631 & Nitrite plus nitrate, water, filtered, milligrams per liter as nitrogen & E0.05 & \\
\hline 00666 & Phosphorus, water, filtered, milligrams per liter & 0.008 & \\
\hline 00665 & Phosphorus, water, unfiltered, milligrams per liter & 0.023 & $0.1^{\mathrm{c}}$ \\
\hline 00680 & Organic carbon, water, unfiltered, milligrams per liter & 8.4 & \\
\hline 00340 & Chemical oxygen demand, high level, water, unfiltered, milligrams per liter & 20 & \\
\hline \multicolumn{4}{|c|}{ Major ions, metals, and related chemistry } \\
\hline 00915 & Calcium, water, filtered, milligrams per liter & 1.77 & \\
\hline 00925 & Magnesium, water, filtered, milligrams per liter & 0.891 & \\
\hline 00935 & Potassium, water, filtered, milligrams per liter & 1.21 & \\
\hline 00930 & Sodium, water, filtered, milligrams per liter & 3.78 & \\
\hline 00940 & Chloride, water, filtered, milligrams per liter & 5.9 & $860(\mathrm{CMC}) / 230(\mathrm{CCC})^{\mathrm{d}}$ \\
\hline 00955 & Silica, water, filtered, milligrams per liter & 12.1 & \\
\hline 00945 & Sulfate, water, filtered, milligrams per liter & 1.5 & \\
\hline 70300 & Residue on evaporation, dried at 180 degrees Celsius, water, filtered, milligrams per liter & 55 & $1,500^{\mathrm{a}}$ \\
\hline 01106 & Aluminum, water, filtered, micrograms per liter & 66 & $750(\mathrm{CMC}) / 87(\mathrm{CCC})^{\mathrm{d}}$ \\
\hline 01000 & Arsenic, water, filtered, micrograms per liter & 0.7 & $340 / 150^{\mathrm{a}}$ \\
\hline 01005 & Barium, water, filtered, micrograms per liter & 38 & $50,000(\text { recom })^{\mathrm{c}}$ \\
\hline 01010 & Beryllium, water, filtered, micrograms per liter & 0.09 & $130 / 5.3^{c}$ \\
\hline 01020 & Boron, water, filtered, micrograms per liter & 12 & \\
\hline 01025 & Cadmium, water, filtered, micrograms per liter & E0.03 & $1.74 / 0.62^{\mathrm{a}}$ \\
\hline 01030 & Chromium, water, filtered, micrograms per liter & 0.3 & $16 / 11^{\mathrm{a}}$ \\
\hline 01035 & Cobalt, water, filtered, micrograms per liter & 0.27 & \\
\hline 01040 & Copper, water, filtered, micrograms per liter & 0.9 & $7 / 5^{\mathrm{a}}$ \\
\hline 01046 & Iron, water, filtered, micrograms per liter & 386 & $1000^{d}$ \\
\hline 01049 & Lead, water, filtered, micrograms per liter & 0.2 & $30 / 1.18^{\mathrm{a}}$ \\
\hline 01130 & Lithium, water, filtered, micrograms per liter & 1.1 & \\
\hline 01056 & Manganese, water, filtered, micrograms per liter & 31.6 & \\
\hline 01065 & Nickel, water, filtered, micrograms per liter & 0.9 & $260 / 29^{a}$ \\
\hline 01145 & Selenium, water, filtered, micrograms per liter & E0.3 & $11.8 / 4.6^{\mathrm{a}}$ \\
\hline 01080 & Strontium, water, filtered, micrograms per liter & 24.2 & \\
\hline 22703 & Uranium (natural), water, filtered, micrograms per liter & E0.03 & \\
\hline 01085 & Vanadium, water, filtered, micrograms per liter & 0.4 & \\
\hline 01090 & Zinc, water, filtered, micrograms per liter & 3 & $65 / 65^{\mathrm{a}}$ \\
\hline \multicolumn{4}{|c|}{ Organic chemicals } \\
\hline 50305 & Caffeine, water, filtered, recoverable, micrograms per liter & E0.106 & \\
\hline 62082 & DEET, water, filtered, recoverable, micrograms per liter & E0.1 & \\
\hline 04025 & Hexazinone, water, filtered, recoverable, micrograms per liter & E0.009 & \\
\hline 39415 & Metolachlor, water, filtered, recoverable, micrograms per liter & E0.005 & \\
\hline 81552 & Acetone, water, unfiltered, recoverable, micrograms per liter & E1 & \\
\hline 34010 & Toluene, water, unfiltered, recoverable, micrograms per liter & E0.08 & $6300 / 5000^{c}$ \\
\hline \multirow{2}{*}{\multicolumn{4}{|c|}{$\begin{array}{l}02479300 \text { Red Creek at Vestry, MS, Sample Date: 9/29/05, Sample Time: } 1030 \\
\text { Physical properties }\end{array}$}} \\
\hline & & & \\
\hline 00061 & Discharge, instantaneous, cubic feet per second & 400 & \\
\hline 63676 & Turbidity, water, unfiltered, Nephlometric Turbidity Ratio-Units & 7 & \\
\hline 00300 & Dissolved oxygen, water, unfiltered, milligrams per liter & 6.6 & 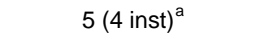 \\
\hline 00400 & $\mathrm{pH}$, water, unfiltered, field, standard units & 5.6 & $6-9^{a}$ \\
\hline 00095 & Specific conductance, water, unfiltered, microsiemens per centimeter at 25 degrees Celsius & 44 & $1000^{\mathrm{a}}$ \\
\hline 00010 & Temperature, water, degrees Celsius & 26.6 & $32.2^{\mathrm{a}}$ \\
\hline
\end{tabular}


Table 8. Continued--Constituents and compounds detected in samples collected in the aftermath of Hurricane Katrina, southeastern Mississippi September 19-30, 2005.

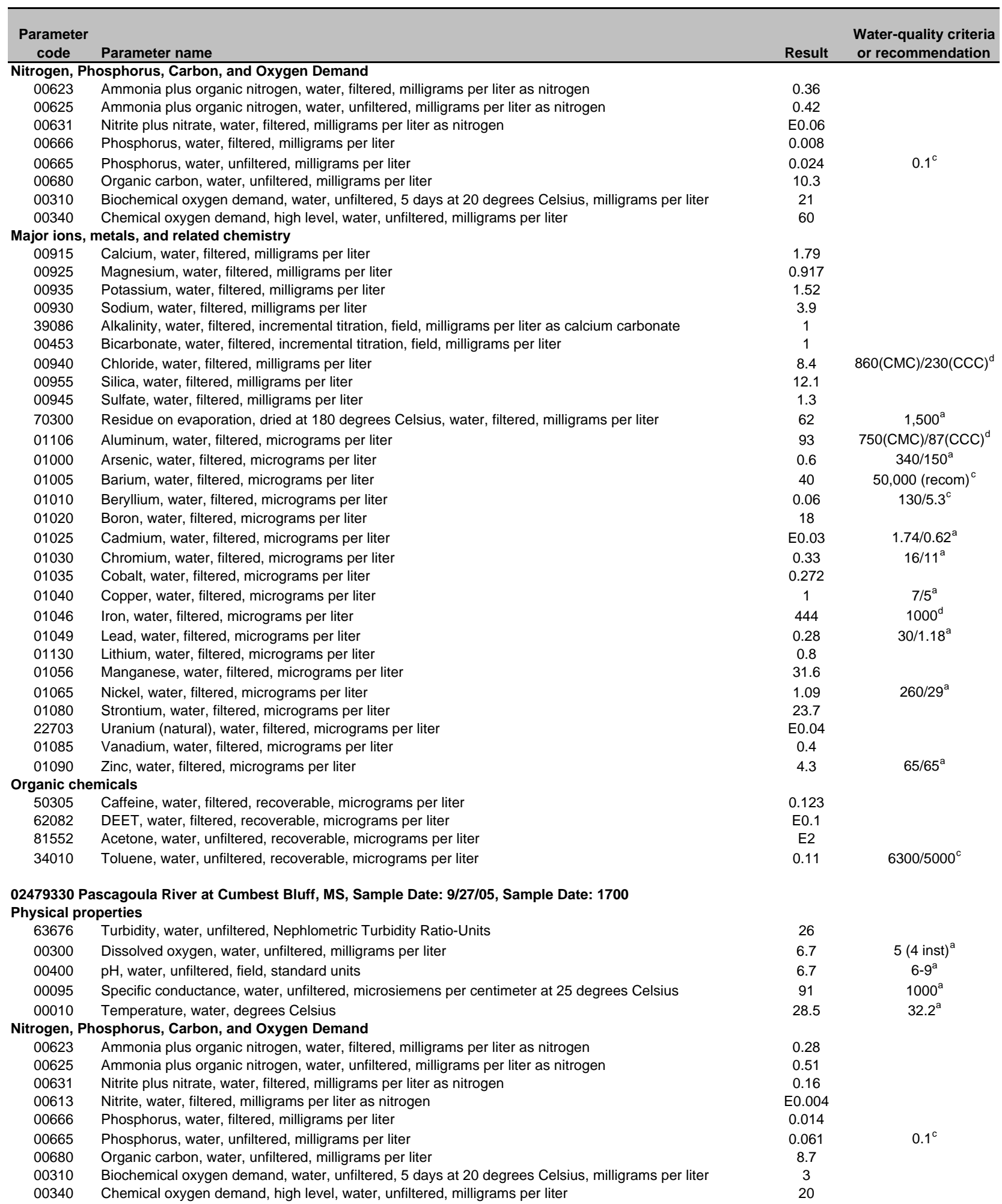


Table 8. Continued--Constituents and compounds detected in samples collected in the aftermath of Hurricane Katrina, southeastern Mississippi September 19-30, 2005.

\begin{tabular}{|c|c|c|c|}
\hline $\begin{array}{l}\text { Parameter } \\
\text { code }\end{array}$ & Parameter name & Result & $\begin{array}{l}\text { Water-quality criteria } \\
\text { or recommendation }\end{array}$ \\
\hline \multicolumn{4}{|c|}{ Major ions, metals, and related chemistry } \\
\hline 00915 & Calcium, water, filtered, milligrams per liter & 4.52 & \\
\hline 00925 & Magnesium, water, filtered, milligrams per liter & 1.23 & \\
\hline 00935 & Potassium, water, filtered, milligrams per liter & 1.85 & \\
\hline 00930 & Sodium, water, filtered, milligrams per liter & 9.77 & \\
\hline 39086 & Alkalinity, water, filtered, incremental titration, field, milligrams per liter as calcium carbonate & 17 & \\
\hline 00453 & Bicarbonate, water, filtered, incremental titration, field, milligrams per liter & 20 & \\
\hline 00940 & Chloride, water, filtered, milligrams per liter & 8.36 & $860(\mathrm{CMC}) / 230(\mathrm{CCC})^{\mathrm{d}}$ \\
\hline 00955 & Silica, water, filtered, milligrams per liter & 11.3 & \\
\hline 00945 & Sulfate, water, filtered, milligrams per liter & 7 & \\
\hline 70300 & Residue on evaporation, dried at 180 degrees Celsius, water, filtered, milligrams per liter & 77 & $1,500^{\mathrm{a}}$ \\
\hline 01106 & Aluminum, water, filtered, micrograms per liter & 32 & $750(\mathrm{CMC}) / 87(\mathrm{CCC})^{\mathrm{d}}$ \\
\hline 01000 & Arsenic, water, filtered, micrograms per liter & 0.7 & $340 / 150^{\mathrm{a}}$ \\
\hline 01005 & Barium, water, filtered, micrograms per liter & 32 & 50,000 (recom) $^{\mathrm{c}}$ \\
\hline 01010 & Beryllium, water, filtered, micrograms per liter & E0.03 & $130 / 5.3^{c}$ \\
\hline 01020 & Boron, water, filtered, micrograms per liter & 23 & \\
\hline 01030 & Chromium, water, filtered, micrograms per liter & 0.19 & $16 / 11^{\mathrm{a}}$ \\
\hline 01035 & Cobalt, water, filtered, micrograms per liter & 0.13 & \\
\hline 01040 & Copper, water, filtered, micrograms per liter & 0.6 & $7 / 5^{\mathrm{a}}$ \\
\hline 01046 & Iron, water, filtered, micrograms per liter & 496 & $1000^{d}$ \\
\hline 01049 & Lead, water, filtered, micrograms per liter & 0.1 & $30 / 1.18^{\mathrm{a}}$ \\
\hline 01130 & Lithium, water, filtered, micrograms per liter & 1 & \\
\hline 01056 & Manganese, water, filtered, micrograms per liter & 43.9 & \\
\hline 01060 & Molybdenum, water, filtered, micrograms per liter & E0.2 & \\
\hline 01065 & Nickel, water, filtered, micrograms per liter & 0.91 & $260 / 29^{a}$ \\
\hline 01080 & Strontium, water, filtered, micrograms per liter & 38.7 & \\
\hline 22703 & Uranium (natural), water, filtered, micrograms per liter & E0.04 & \\
\hline 01085 & Vanadium, water, filtered, micrograms per liter & 0.4 & \\
\hline 01090 & Zinc, water, filtered, micrograms per liter & 1.4 & $65 / 65^{\mathrm{a}}$ \\
\hline \multicolumn{4}{|c|}{ Organic chemicals } \\
\hline 50305 & Caffeine, water, filtered, recoverable, micrograms per liter & 0.03 & \\
\hline 04025 & Hexazinone, water, filtered, recoverable, micrograms per liter & E0.009 & \\
\hline 34443 & Naphthalene, water, filtered, recoverable, micrograms per liter & E0.1 & $2300 / 620^{c}$ \\
\hline 34010 & Toluene, water, unfiltered, recoverable, micrograms per liter & E0.03 & $6300 / 5000^{c}$ \\
\hline \multicolumn{4}{|c|}{02479560 Escatawpa River near Agricola, MS, Sample Date: 9/20/05, Sample Time: 1045} \\
\hline \multicolumn{4}{|c|}{ Physical properties } \\
\hline 00061 & Discharge, instantaneous, cubic feet per second & 343 & \\
\hline 00300 & Dissolved oxygen, water, unfiltered, milligrams per liter & 6.6 & 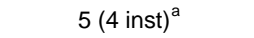 \\
\hline 00400 & $\mathrm{pH}$, water, unfiltered, field, standard units & 5.5 & $6-9^{a}$ \\
\hline 00095 & Specific conductance, water, unfiltered, microsiemens per centimeter at 25 degrees Celsius & 30 & $1000^{\mathrm{a}}$ \\
\hline 00010 & Temperature, water, degrees Celsius & 26.6 & $32.2^{\mathrm{a}}$ \\
\hline \multicolumn{4}{|c|}{ Nitrogen, Phosphorus, Carbon, and Oxygen Demand } \\
\hline 00623 & Ammonia plus organic nitrogen, water, filtered, milligrams per liter as nitrogen & 0.47 & \\
\hline 00625 & Ammonia plus organic nitrogen, water, unfiltered, milligrams per liter as nitrogen & 0.4 & \\
\hline 00608 & Ammonia, water, filtered, milligrams per liter as nitrogen & E0.03 & $1.5-2^{b}$ \\
\hline 00631 & Nitrite plus nitrate, water, filtered, milligrams per liter as nitrogen & 0.1 & \\
\hline 00666 & Phosphorus, water, filtered, milligrams per liter & E0.003 & \\
\hline 00665 & Phosphorus, water, unfiltered, milligrams per liter & 0.014 & $0.1^{\mathrm{c}}$ \\
\hline 00680 & Organic carbon, water, unfiltered, milligrams per liter & 9.5 & \\
\hline 00340 & Chemical oxygen demand, high level, water, unfiltered, milligrams per liter & 20 & \\
\hline \multicolumn{4}{|c|}{ Major ions, metals, and related chemistry } \\
\hline 00915 & Calcium, water, filtered, milligrams per liter & 1.29 & \\
\hline 00925 & Magnesium, water, filtered, milligrams per liter & 0.848 & \\
\hline 00935 & Potassium, water, filtered, milligrams per liter & 0.79 & \\
\hline 00930 & Sodium, water, filtered, milligrams per liter & 2.32 & \\
\hline 00940 & Chloride, water, filtered, milligrams per liter & 4.33 & $860(\mathrm{CMC}) / 230(\mathrm{CCC})^{d}$ \\
\hline 00955 & Silica, water, filtered, milligrams per liter & 9.3 & \\
\hline 00945 & Sulfate, water, filtered, milligrams per liter & 1.4 & \\
\hline 70300 & Residue on evaporation, dried at 180 degrees Celsius, water, filtered, milligrams per liter & 38 & $1,500^{\mathrm{a}}$ \\
\hline 01106 & Aluminum, water, filtered, micrograms per liter & 102 & $750(\mathrm{CMC}) / 87(\mathrm{CCC})^{\mathrm{d}}$ \\
\hline 01000 & Arsenic, water, filtered, micrograms per liter & 0.8 & $340 / 150^{a}$ \\
\hline 01005 & Barium, water, filtered, micrograms per liter & 33 & $50,000(\text { recom })^{c}$ \\
\hline 01010 & Beryllium, water, filtered, micrograms per liter & E0.05 & $130 / 5.3^{c}$ \\
\hline 01020 & Boron, water, filtered, micrograms per liter & 11 & \\
\hline
\end{tabular}


Table 8. Continued--Constituents and compounds detected in samples collected in the aftermath of Hurricane Katrina, southeastern Mississippi September 19-30, 2005.

\begin{tabular}{|c|c|c|c|}
\hline $\begin{array}{c}\text { Parameter } \\
\text { code }\end{array}$ & Parameter name & Result & $\begin{array}{l}\text { Water-quality criteria } \\
\text { or recommendation }\end{array}$ \\
\hline 01030 & Chromium, water, filtered, micrograms per liter & 0.3 & $16 / 11^{\mathrm{a}}$ \\
\hline 01035 & Cobalt, water, filtered, micrograms per liter & 0.756 & \\
\hline 01040 & Copper, water, filtered, micrograms per liter & 0.8 & $7 / 5^{\mathrm{a}}$ \\
\hline 01046 & Iron, water, filtered, micrograms per liter & 477 & $1000^{d}$ \\
\hline 01049 & Lead, water, filtered, micrograms per liter & 0.24 & $30 / 1.18^{\mathrm{a}}$ \\
\hline 01130 & Lithium, water, filtered, micrograms per liter & 0.7 & \\
\hline 01056 & Manganese, water, filtered, micrograms per liter & 41.1 & \\
\hline 01065 & Nickel, water, filtered, micrograms per liter & 1.19 & $260 / 29^{a}$ \\
\hline 01145 & Selenium, water, filtered, micrograms per liter & E0.2 & $11.8 / 4.6^{\mathrm{a}}$ \\
\hline 01080 & Strontium, water, filtered, micrograms per liter & 17.1 & \\
\hline 22703 & Uranium (natural), water, filtered, micrograms per liter & E0.03 & \\
\hline 01085 & Vanadium, water, filtered, micrograms per liter & 0.4 & \\
\hline 01090 & Zinc, water, filtered, micrograms per liter & 3.5 & $65 / 65^{\mathrm{a}}$ \\
\hline \multicolumn{4}{|c|}{ Organic chemicals } \\
\hline 39415 & Metolachlor, water, filtered, recoverable, micrograms per liter & E0.005 & \\
\hline 82670 & Tebuthiuron, water, filtered ( 0.7 micron glass fiber filter), recoverable, micrograms per liter & 0.02 & \\
\hline 81552 & Acetone, water, unfiltered, recoverable, micrograms per liter & E3 & \\
\hline 34010 & Toluene, water, unfiltered, recoverable, micrograms per liter & E0.05 & $6300 / 5000^{c}$ \\
\hline \multirow{2}{*}{\multicolumn{4}{|c|}{$\begin{array}{l}02480254 \text { Bluff Creek at Vancleave, MS, Sampling Date: 9/20/05, Sample Time: } 1745 \\
\text { Physical properties }\end{array}$}} \\
\hline & & & \\
\hline 00300 & Dissolved oxygen, water, unfiltered, milligrams per liter & 6.2 & 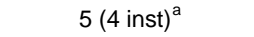 \\
\hline 00400 & $\mathrm{pH}$, water, unfiltered, field, standard units & 5.3 & $6-9^{a}$ \\
\hline 00095 & Specific conductance, water, unfiltered, microsiemens per centimeter at 25 degrees Celsius & 33 & $1000^{\mathrm{a}}$ \\
\hline 00010 & Temperature, water, degrees Celsius & 27.5 & $32.2^{\mathrm{a}}$ \\
\hline \multicolumn{4}{|c|}{ Nitrogen, Phosphorus, Carbon, and Oxygen Demand } \\
\hline 00623 & Ammonia plus organic nitrogen, water, filtered, milligrams per liter as nitrogen & 0.3 & \\
\hline 00625 & Ammonia plus organic nitrogen, water, unfiltered, milligrams per liter as nitrogen & 0.36 & \\
\hline 00608 & Ammonia, water, filtered, milligrams per liter as nitrogen & E0.03 & $1.5-2^{b}$ \\
\hline 00666 & Phosphorus, water, filtered, milligrams per liter & E0.003 & \\
\hline 00340 & Chemical oxygen demand, high level, water, unfiltered, milligrams per liter & 30 & \\
\hline \multicolumn{4}{|c|}{ Major ions, metals, and related chemistry } \\
\hline 00915 & Calcium, water, filtered, milligrams per liter & 1.05 & \\
\hline 00925 & Magnesium, water, filtered, milligrams per liter & 0.612 & \\
\hline 00935 & Potassium, water, filtered, milligrams per liter & 1.06 & \\
\hline 00930 & Sodium, water, filtered, milligrams per liter & 3.49 & \\
\hline 00940 & Chloride, water, filtered, milligrams per liter & 5.58 & $860(\mathrm{CMC}) / 230(\mathrm{CCC})^{d}$ \\
\hline 00955 & Silica, water, filtered, milligrams per liter & 13.5 & \\
\hline 00945 & Sulfate, water, filtered, milligrams per liter & 1.2 & \\
\hline 70300 & Residue on evaporation, dried at 180 degrees Celsius, water, filtered, milligrams per liter & 42 & $1,500^{\mathrm{a}}$ \\
\hline 01106 & Aluminum, water, filtered, micrograms per liter & 116 & $750(\mathrm{CMC}) / 87(\mathrm{CCC})^{\mathrm{d}}$ \\
\hline 01000 & Arsenic, water, filtered, micrograms per liter & 0.8 & $340 / 150^{\mathrm{a}}$ \\
\hline 01005 & Barium, water, filtered, micrograms per liter & 34 & $50,000(\text { recom })^{c}$ \\
\hline 01010 & Beryllium, water, filtered, micrograms per liter & 0.08 & $130 / 5.3^{c}$ \\
\hline 01020 & Boron, water, filtered, micrograms per liter & 12 & \\
\hline 01025 & Cadmium, water, filtered, micrograms per liter & 0.07 & $1.74 / 0.62^{\mathrm{a}}$ \\
\hline 01030 & Chromium, water, filtered, micrograms per liter & 0.37 & $16 / 11^{\mathrm{a}}$ \\
\hline 01035 & Cobalt, water, filtered, micrograms per liter & 1.26 & \\
\hline 01040 & Copper, water, filtered, micrograms per liter & 0.5 & $7 / 5^{\mathrm{a}}$ \\
\hline 01046 & Iron, water, filtered, micrograms per liter & 361 & $1000^{d}$ \\
\hline 01049 & Lead, water, filtered, micrograms per liter & 0.17 & $30 / 1.18^{\mathrm{a}}$ \\
\hline 01130 & Lithium, water, filtered, micrograms per liter & 0.8 & \\
\hline 01056 & Manganese, water, filtered, micrograms per liter & 45.4 & \\
\hline 01065 & Nickel, water, filtered, micrograms per liter & 1.03 & $260 / 29^{a}$ \\
\hline 01145 & Selenium, water, filtered, micrograms per liter & E0.2 & $11.8 / 4.6^{\mathrm{a}}$ \\
\hline 01080 & Strontium, water, filtered, micrograms per liter & 10.6 & \\
\hline 01057 & Thallium, water, filtered, micrograms per liter & E0.02 & $1400 / 40^{c}$ \\
\hline 22703 & Uranium (natural), water, filtered, micrograms per liter & E0.03 & \\
\hline 01085 & Vanadium, water, filtered, micrograms per liter & 0.3 & \\
\hline 01090 & Zinc, water, filtered, micrograms per liter & 5.7 & $65 / 65^{a}$ \\
\hline
\end{tabular}


Table 8. Continued--Constituents and compounds detected in samples collected in the aftermath of Hurricane Katrina, southeastern Mississippi September 19-30, 2005.

\begin{tabular}{|c|c|c|c|}
\hline $\begin{array}{l}\text { Parameter } \\
\text { code }\end{array}$ & Parameter name & Result & $\begin{array}{l}\text { Water-quality criteria } \\
\text { or recommendation }\end{array}$ \\
\hline \multicolumn{4}{|c|}{ Organic chemicals } \\
\hline 62085 & 4-Nonylphenol, water, filtered, recoverable, micrograms per liter & E2 & \\
\hline 50305 & Caffeine, water, filtered, recoverable, micrograms per liter & E0.147 & \\
\hline 62082 & DEET, water, filtered, recoverable, micrograms per liter & E0.2 & \\
\hline 62083 & Diethoxynonylphenol, water, filtered, recoverable, micrograms per liter & E2 & \\
\hline 34466 & Phenol, water, filtered, recoverable, micrograms per liter & E0.9 & $300 / 102^{\mathrm{a}}$ \\
\hline 81552 & Acetone, water, unfiltered, recoverable, micrograms per liter & E4 & \\
\hline 34010 & Toluene, water, unfiltered, recoverable, micrograms per liter & 0.48 & $6300 / 5000^{c}$ \\
\hline \multicolumn{4}{|c|}{02480254 Bluff Creek at Vancleave, MS, Sampling Date: 9/27/05, Sample Time: 2030} \\
\hline \multicolumn{4}{|c|}{ Physical properties } \\
\hline 63676 & Turbidity, water, unfiltered, Nephlometric Turbidity Ratio-Units & 6 & \\
\hline 00300 & Dissolved oxygen, water, unfiltered, milligrams per liter & 6.8 & $5(4 \text { inst })^{a}$ \\
\hline 00400 & $\mathrm{pH}$, water, unfiltered, field, standard units & 5.1 & $6-9^{a}$ \\
\hline 00095 & Specific conductance, water, unfiltered, microsiemens per centimeter at 25 degrees Celsius & 36 & $1000^{\mathrm{a}}$ \\
\hline 00010 & Temperature, water, degrees Celsius & 27.5 & $32.2^{\mathrm{a}}$ \\
\hline \multicolumn{4}{|c|}{ Nitrogen, Phosphorus, Carbon, and Oxygen Demand } \\
\hline 00623 & Ammonia plus organic nitrogen, water, filtered, milligrams per liter as nitrogen & 0.31 & \\
\hline 00625 & Ammonia plus organic nitrogen, water, unfiltered, milligrams per liter as nitrogen & 0.33 & \\
\hline 00608 & Ammonia, water, filtered, milligrams per liter as nitrogen & E0.03 & $1.5-2^{b}$ \\
\hline 00665 & Phosphorus, water, unfiltered, milligrams per liter & 0.01 & $0.1^{\mathrm{c}}$ \\
\hline 00680 & Organic carbon, water, unfiltered, milligrams per liter & 10.9 & \\
\hline 00340 & Chemical oxygen demand, high level, water, unfiltered, milligrams per liter & 20 & \\
\hline \multicolumn{4}{|c|}{ Major ions, metals, and related chemistry } \\
\hline 00915 & Calcium, water, filtered, milligrams per liter & 0.98 & \\
\hline 00925 & Magnesium, water, filtered, milligrams per liter & 0.637 & \\
\hline 00935 & Potassium, water, filtered, milligrams per liter & 1.15 & \\
\hline 00930 & Sodium, water, filtered, milligrams per liter & 3.54 & \\
\hline 39086 & Alkalinity, water, filtered, incremental titration, field, milligrams per liter as calcium carbonate & 1 & \\
\hline 00453 & Bicarbonate, water, filtered, incremental titration, field, milligrams per liter & 1 & \\
\hline 00940 & Chloride, water, filtered, milligrams per liter & 6.46 & $860(\mathrm{CMC}) / 230(\mathrm{CCC})^{\mathrm{d}}$ \\
\hline 00955 & Silica, water, filtered, milligrams per liter & 12.9 & \\
\hline 00945 & Sulfate, water, filtered, milligrams per liter & 1.1 & \\
\hline 70300 & Residue on evaporation, dried at 180 degrees Celsius, water, filtered, milligrams per liter & 48 & $1,500^{\mathrm{a}}$ \\
\hline 01106 & Aluminum, water, filtered, micrograms per liter & 151 & $750(\mathrm{CMC}) / 87(\mathrm{CCC})^{d}$ \\
\hline 01000 & Arsenic, water, filtered, micrograms per liter & 0.8 & $340 / 150^{a}$ \\
\hline 01005 & Barium, water, filtered, micrograms per liter & 36 & $50,000(\text { recom })^{c}$ \\
\hline 01010 & Beryllium, water, filtered, micrograms per liter & 0.1 & $130 / 5.3^{c}$ \\
\hline 01020 & Boron, water, filtered, micrograms per liter & 16 & \\
\hline 01025 & Cadmium, water, filtered, micrograms per liter & 0.11 & $1.74 / 0.62^{\mathrm{a}}$ \\
\hline 01030 & Chromium, water, filtered, micrograms per liter & 0.39 & $16 / 11^{\mathrm{a}}$ \\
\hline 01035 & Cobalt, water, filtered, micrograms per liter & 1.26 & \\
\hline 01040 & Copper, water, filtered, micrograms per liter & 13.1 & $7 / 5^{\mathrm{a}}$ \\
\hline 01046 & Iron, water, filtered, micrograms per liter & 305 & $1000^{d}$ \\
\hline 01049 & Lead, water, filtered, micrograms per liter & 0.59 & $30 / 1.18^{\mathrm{a}}$ \\
\hline 01130 & Lithium, water, filtered, micrograms per liter & 0.7 & \\
\hline 01056 & Manganese, water, filtered, micrograms per liter & 42.6 & \\
\hline 01065 & Nickel, water, filtered, micrograms per liter & 1.52 & $260 / 29^{a}$ \\
\hline 01080 & Strontium, water, filtered, micrograms per liter & 10.1 & \\
\hline 01057 & Thallium, water, filtered, micrograms per liter & E0.03 & $1400 / 40^{c}$ \\
\hline 22703 & Uranium (natural), water, filtered, micrograms per liter & E0.03 & \\
\hline 01085 & Vanadium, water, filtered, micrograms per liter & 0.3 & \\
\hline 01090 & Zinc, water, filtered, micrograms per liter & 21.5 & $65 / 65^{\mathrm{a}}$ \\
\hline \multicolumn{4}{|c|}{ Organic chemicals } \\
\hline 50305 & Caffeine, water, filtered, recoverable, micrograms per liter & E0.1 & \\
\hline 62083 & Diethoxynonylphenol, water, filtered, recoverable, micrograms per liter & E3 & \\
\hline 77356 & 4-Isopropyltoluene, water, unfiltered, recoverable, micrograms per liter & E0.02 & \\
\hline 81552 & Acetone, water, unfiltered, recoverable, micrograms per liter & E3 & \\
\hline 77128 & Styrene, water, unfiltered, recoverable, micrograms per liter & E0.02 & \\
\hline 34010 & Toluene, water, unfiltered, recoverable, micrograms per liter & 1.27 & $6300 / 5000^{c}$ \\
\hline
\end{tabular}


Table 8. Continued--Constituents and compounds detected in samples collected in the aftermath of Hurricane Katrina, southeastern Mississippi September 19-30, 2005.

\begin{tabular}{|c|c|c|c|}
\hline $\begin{array}{l}\text { Parameter } \\
\text { code }\end{array}$ & Parameter name & Result & $\begin{array}{l}\text { Water-quality criteria } \\
\text { or recommendation }\end{array}$ \\
\hline \multicolumn{4}{|c|}{02480599 Tchoutacabouffa River at D'Iberville, MS, Sample Date: 9/21/05, Sample Time: 1030} \\
\hline \multicolumn{4}{|c|}{ Physical properties } \\
\hline 00300 & Dissolved oxygen, water, unfiltered, milligrams per liter & 3.3 & 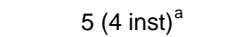 \\
\hline 00400 & $\mathrm{pH}$, water, unfiltered, field, standard units & 5.1 & $6-9^{a}$ \\
\hline 00095 & Specific conductance, water, unfiltered, microsiemens per centimeter at 25 degrees Celsius & 72 & $1000^{\mathrm{a}}$ \\
\hline 00010 & Temperature, water, degrees Celsius & 29.1 & $32.2^{\mathrm{a}}$ \\
\hline \multicolumn{4}{|c|}{ Nitrogen, Phosphorus, Carbon, and Oxygen Demand } \\
\hline 00623 & Ammonia plus organic nitrogen, water, filtered, milligrams per liter as nitrogen & 0.42 & \\
\hline 00625 & Ammonia plus organic nitrogen, water, unfiltered, milligrams per liter as nitrogen & 0.52 & \\
\hline 00666 & Phosphorus, water, filtered, milligrams per liter & 0.006 & \\
\hline 00665 & Phosphorus, water, unfiltered, milligrams per liter & 0.037 & $0.1^{\mathrm{c}}$ \\
\hline 00680 & Organic carbon, water, unfiltered, milligrams per liter & 13.1 & \\
\hline 00340 & Chemical oxygen demand, high level, water, unfiltered, milligrams per liter & 30 & \\
\hline \multicolumn{4}{|c|}{ Major ions, metals, and related chemistry } \\
\hline 00915 & Calcium, water, filtered, milligrams per liter & 1.71 & \\
\hline 00935 & Potassium, water, filtered, milligrams per liter & 1.18 & \\
\hline 00930 & Sodium, water, filtered, milligrams per liter & 7.11 & \\
\hline 00940 & Chloride, water, filtered, milligrams per liter & 11.5 & $860(\mathrm{CMC}) / 230(\mathrm{CCC})^{\mathrm{d}}$ \\
\hline 00955 & Silica, water, filtered, milligrams per liter & 12.7 & \\
\hline 00945 & Sulfate, water, filtered, milligrams per liter & 2.4 & \\
\hline 70300 & Residue on evaporation, dried at 180 degrees Celsius, water, filtered, milligrams per liter & 73 & $1,500^{\mathrm{a}}$ \\
\hline 01106 & Aluminum, water, filtered, micrograms per liter & 156 & $750(\mathrm{CMC}) / 87(\mathrm{CCC})^{\mathrm{d}}$ \\
\hline 01000 & Arsenic, water, filtered, micrograms per liter & 1.1 & $340 / 150^{a}$ \\
\hline 01005 & Barium, water, filtered, micrograms per liter & 37 & $50,000(\text { recom })^{c}$ \\
\hline 01010 & Beryllium, water, filtered, micrograms per liter & 0.11 & $130 / 5.3^{c}$ \\
\hline 01020 & Boron, water, filtered, micrograms per liter & 20 & \\
\hline 01025 & Cadmium, water, filtered, micrograms per liter & 0.05 & $1.74 / 0.62^{\mathrm{a}}$ \\
\hline 01030 & Chromium, water, filtered, micrograms per liter & 0.47 & $16 / 11^{\mathrm{a}}$ \\
\hline 01035 & Cobalt, water, filtered, micrograms per liter & 1.36 & \\
\hline 01040 & Copper, water, filtered, micrograms per liter & 1 & $7 / 5^{a}$ \\
\hline 01046 & Iron, water, filtered, micrograms per liter & 345 & $1000^{d}$ \\
\hline 01056 & Manganese, water, filtered, micrograms per liter & 73.4 & \\
\hline 01065 & Nickel, water, filtered, micrograms per liter & 1.35 & $260 / 29^{a}$ \\
\hline 01080 & Strontium, water, filtered, micrograms per liter & 20 & \\
\hline 22703 & Uranium (natural), water, filtered, micrograms per liter & 0.04 & \\
\hline 01085 & Vanadium, water, filtered, micrograms per liter & 0.3 & \\
\hline 01090 & Zinc, water, filtered, micrograms per liter & 4.8 & $65 / 65^{\mathrm{a}}$ \\
\hline \multicolumn{4}{|c|}{ Organic chemicals } \\
\hline 39632 & Atrazine, water, filtered, recoverable, micrograms per liter & E0.007 & $1,500^{\mathrm{e}}$ \\
\hline 50305 & Caffeine, water, filtered, recoverable, micrograms per liter & E0.111 & \\
\hline 62082 & DEET, water, filtered, recoverable, micrograms per liter & E0.1 & \\
\hline 34466 & Phenol, water, filtered, recoverable, micrograms per liter & E0.1 & $300 / 102^{a}$ \\
\hline 77356 & 4-Isopropyltoluene, water, unfiltered, recoverable, micrograms per liter & E0.03 & \\
\hline 81552 & Acetone, water, unfiltered, recoverable, micrograms per liter & E2 & \\
\hline 34010 & Toluene, water, unfiltered, recoverable, micrograms per liter & 0.31 & $6300 / 5000^{c}$ \\
\hline 38775 & Dichlorvos, water, filtered, recoverable, micrograms per liter & E0.03 & \\
\hline \multicolumn{4}{|c|}{02480599 Tchoutacabouffa River at D'Iberville, MS, Sample Date: 9/28/05, Sample Time: 0930} \\
\hline \multicolumn{4}{|c|}{ Physical properties } \\
\hline 63676 & Turbidity, water, unfiltered, Nephlometric Turbidity Ratio-Units & 5 & \\
\hline 00300 & Dissolved oxygen, water, unfiltered, milligrams per liter & 3.6 & 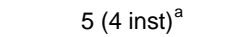 \\
\hline 00400 & $\mathrm{pH}$, water, unfiltered, field, standard units & 5.3 & $6-9^{a}$ \\
\hline 00095 & Specific conductance, water, unfiltered, microsiemens per centimeter at 25 degrees Celsius & 79 & $1000^{\mathrm{a}}$ \\
\hline 00010 & Temperature, water, degrees Celsius & 28 & $32.2^{\mathrm{a}}$ \\
\hline \multicolumn{4}{|c|}{ Nitrogen, Phosphorus, Carbon, and Oxygen Demand } \\
\hline 00623 & Ammonia plus organic nitrogen, water, filtered, milligrams per liter as nitrogen & 0.6 & \\
\hline 00625 & Ammonia plus organic nitrogen, water, unfiltered, milligrams per liter as nitrogen & 0.51 & \\
\hline 00666 & Phosphorus, water, filtered, milligrams per liter & 0.017 & \\
\hline 00665 & Phosphorus, water, unfiltered, milligrams per liter & 0.044 & $0.1^{\mathrm{c}}$ \\
\hline 00680 & Organic carbon, water, unfiltered, milligrams per liter & 15.4 & \\
\hline 00310 & Biochemical oxygen demand, water, unfiltered, 5 days at 20 degrees Celsius, milligrams per liter & 11 & \\
\hline 00340 & Chemical oxygen demand, high level, water, unfiltered, milligrams per liter & 50 & \\
\hline
\end{tabular}


Table 8. Continued--Constituents and compounds detected in samples collected in the aftermath of Hurricane Katrina, southeastern Mississippi September 19-30, 2005.

\begin{tabular}{|c|c|c|c|}
\hline $\begin{array}{c}\text { Parameter } \\
\text { code }\end{array}$ & Parameter name & Result & $\begin{array}{l}\text { Water-quality criteria } \\
\text { or recommendation }\end{array}$ \\
\hline \multicolumn{4}{|c|}{ Major ions, metals, and related chemistry } \\
\hline 00915 & Calcium, water, filtered, milligrams per liter & 1.78 & \\
\hline 00925 & Magnesium, water, filtered, milligrams per liter & 1.1 & \\
\hline 00935 & Potassium, water, filtered, milligrams per liter & 1.82 & \\
\hline 00930 & Sodium, water, filtered, milligrams per liter & 9.5 & \\
\hline 39086 & Alkalinity, water, filtered, incremental titration, field, milligrams per liter as calcium carbonate & 3 & \\
\hline 00453 & Bicarbonate, water, filtered, incremental titration, field, milligrams per liter & 4 & \\
\hline 00940 & Chloride, water, filtered, milligrams per liter & 14.4 & $860(\mathrm{CMC}) / 230(\mathrm{CCC})^{\mathrm{d}}$ \\
\hline 00955 & Silica, water, filtered, milligrams per liter & 11.7 & \\
\hline 00945 & Sulfate, water, filtered, milligrams per liter & 3.3 & \\
\hline 70300 & Residue on evaporation, dried at 180 degrees Celsius, water, filtered, milligrams per liter & 87 & $1,500^{\mathrm{a}}$ \\
\hline 01106 & Aluminum, water, filtered, micrograms per liter & 167 & $750(\mathrm{CMC}) / 87(\mathrm{CCC})^{\mathrm{d}}$ \\
\hline 01000 & Arsenic, water, filtered, micrograms per liter & 0.9 & $340 / 150^{\mathrm{a}}$ \\
\hline 01005 & Barium, water, filtered, micrograms per liter & 36 & $50,000(\text { recom })^{c}$ \\
\hline 01010 & Beryllium, water, filtered, micrograms per liter & 0.11 & $130 / 5.3^{c}$ \\
\hline 01020 & Boron, water, filtered, micrograms per liter & 33 & \\
\hline 01025 & Cadmium, water, filtered, micrograms per liter & E0.02 & $1.74 / 0.62^{\mathrm{a}}$ \\
\hline 01030 & Chromium, water, filtered, micrograms per liter & 0.4 & $16 / 11^{\mathrm{a}}$ \\
\hline 01035 & Cobalt, water, filtered, micrograms per liter & 1.3 & \\
\hline 01040 & Copper, water, filtered, micrograms per liter & 1.1 & $7 / 5^{\mathrm{a}}$ \\
\hline 01046 & Iron, water, filtered, micrograms per liter & 354 & $1000^{d}$ \\
\hline 01049 & Lead, water, filtered, micrograms per liter & 0.34 & $30 / 1.18^{\mathrm{a}}$ \\
\hline 01130 & Lithium, water, filtered, micrograms per liter & 1.1 & \\
\hline 01056 & Manganese, water, filtered, micrograms per liter & 77.5 & \\
\hline 01065 & Nickel, water, filtered, micrograms per liter & 1.25 & $260 / 29^{a}$ \\
\hline 01080 & Strontium, water, filtered, micrograms per liter & 20.2 & \\
\hline 01057 & Thallium, water, filtered, micrograms per liter & E0.03 & $1400 / 40^{\mathrm{C}}$ \\
\hline 22703 & Uranium (natural), water, filtered, micrograms per liter & 0.05 & \\
\hline 01085 & Vanadium, water, filtered, micrograms per liter & 0.3 & \\
\hline 01090 & Zinc, water, filtered, micrograms per liter & 5.4 & $65 / 65^{\mathrm{a}}$ \\
\hline \multicolumn{4}{|c|}{ Organic chemicals } \\
\hline 50305 & Caffeine, water, filtered, recoverable, micrograms per liter & 0.075 & \\
\hline 62072 & Cholesterol, water, filtered, recoverable, micrograms per liter & E1 & \\
\hline 62082 & DEET, water, filtered, recoverable, micrograms per liter & E0.1 & \\
\hline 62083 & Diethoxynonylphenol, water, filtered, recoverable, micrograms per liter & E2 & \\
\hline 62167 & Fipronil sulfide, water, filtered, recoverable, micrograms per liter & E0.005 & \\
\hline \multicolumn{4}{|c|}{02481000 Biloxi River at Wortham, MS, Sample Date: 9/19/05, Sample Time: 1630} \\
\hline \multicolumn{4}{|c|}{ Physical properties } \\
\hline 00061 & Discharge, instantaneous, cubic feet per second & 60 & \\
\hline 00300 & Dissolved oxygen, water, unfiltered, milligrams per liter & 6.7 & 5 (4 inst) $^{\mathrm{a}}$ \\
\hline 00400 & $\mathrm{pH}$, water, unfiltered, field, standard units & 5.3 & $6-9^{\mathrm{a}}$ \\
\hline 00095 & Specific conductance, water, unfiltered, microsiemens per centimeter at 25 degrees Celsius & 35 & $1000^{\mathrm{a}}$ \\
\hline 00010 & Temperature, water, degrees Celsius & 26.8 & $32.2^{\mathrm{a}}$ \\
\hline \multicolumn{4}{|c|}{ Nitrogen, Phosphorus, Carbon, and Oxygen Demand } \\
\hline 00623 & Ammonia plus organic nitrogen, water, filtered, milligrams per liter as nitrogen & 0.39 & \\
\hline 00625 & Ammonia plus organic nitrogen, water, unfiltered, milligrams per liter as nitrogen & 0.49 & \\
\hline 00666 & Phosphorus, water, filtered, milligrams per liter & E0.003 & \\
\hline 00665 & Phosphorus, water, unfiltered, milligrams per liter & 0.024 & $0.1^{\mathrm{c}}$ \\
\hline 00680 & Organic carbon, water, unfiltered, milligrams per liter & 12.7 & \\
\hline 00340 & Chemical oxygen demand, high level, water, unfiltered, milligrams per liter & 30 & \\
\hline \multicolumn{4}{|c|}{ Major ions, metals, and related chemistry } \\
\hline 00915 & Calcium, water, filtered, milligrams per liter & 1.54 & \\
\hline 00925 & Magnesium, water, filtered, milligrams per liter & 0.928 & \\
\hline 00935 & Potassium, water, filtered, milligrams per liter & 1.16 & \\
\hline 00930 & Sodium, water, filtered, milligrams per liter & 3.23 & \\
\hline 00940 & Chloride, water, filtered, milligrams per liter & 5.1 & $860(\mathrm{CMC}) / 230(\mathrm{CCC})^{d}$ \\
\hline 00955 & Silica, water, filtered, milligrams per liter & 15.7 & \\
\hline 00945 & Sulfate, water, filtered, milligrams per liter & 1.9 & \\
\hline 70300 & Residue on evaporation, dried at 180 degrees Celsius, water, filtered, milligrams per liter & 54 & $1,500^{\mathrm{a}}$ \\
\hline 01106 & Aluminum, water, filtered, micrograms per liter & 136 & $750(\mathrm{CMC}) / 87(\mathrm{CCC})^{d}$ \\
\hline 01000 & Arsenic, water, filtered, micrograms per liter & 1 & $340 / 150^{\mathrm{a}}$ \\
\hline 01005 & Barium, water, filtered, micrograms per liter & 54 & $50,000(\text { recom })^{c}$ \\
\hline 01010 & Beryllium, water, filtered, micrograms per liter & 0.14 & $130 / 5.3^{c}$ \\
\hline 01020 & Boron, water, filtered, micrograms per liter & 9 & \\
\hline 01030 & Chromium, water, filtered, micrograms per liter & 0.52 & $16 / 11^{\mathrm{a}}$ \\
\hline
\end{tabular}


Table 8. Continued--Constituents and compounds detected in samples collected in the aftermath of Hurricane Katrina, southeastern Mississippi September 19-30, 2005.

\begin{tabular}{|c|c|c|c|}
\hline $\begin{array}{c}\text { Parameter } \\
\text { code }\end{array}$ & Parameter name & Result & $\begin{array}{l}\text { Water-quality criteria } \\
\text { or recommendation }\end{array}$ \\
\hline 01035 & Cobalt, water, filtered, micrograms per liter & 0.778 & \\
\hline 01040 & Copper, water, filtered, micrograms per liter & 0.7 & $7 / 5^{a}$ \\
\hline 01046 & Iron, water, filtered, micrograms per liter & 499 & $1000^{d}$ \\
\hline 01049 & Lead, water, filtered, micrograms per liter & 0.24 & $30 / 1.18^{a}$ \\
\hline 01130 & Lithium, water, filtered, micrograms per liter & 1.2 & \\
\hline 01056 & Manganese, water, filtered, micrograms per liter & 50.2 & \\
\hline 01065 & Nickel, water, filtered, micrograms per liter & 1.68 & $260 / 29^{a}$ \\
\hline 01145 & Selenium, water, filtered, micrograms per liter & 0.5 & $11.8 / 4.6^{\mathrm{a}}$ \\
\hline 01080 & Strontium, water, filtered, micrograms per liter & 25.7 & \\
\hline 22703 & Uranium (natural), water, filtered, micrograms per liter & 0.06 & \\
\hline 01085 & Vanadium, water, filtered, micrograms per liter & 0.4 & \\
\hline 01090 & Zinc, water, filtered, micrograms per liter & 2.7 & $65 / 65^{\mathrm{a}}$ \\
\hline \multicolumn{4}{|c|}{ Organic chemicals } \\
\hline 62085 & 4-Nonylphenol, water, filtered, recoverable, micrograms per liter & E1 & \\
\hline 50305 & Caffeine, water, filtered, recoverable, micrograms per liter & E0.097 & \\
\hline 81552 & Acetone, water, unfiltered, recoverable, micrograms per liter & E3 & \\
\hline 34010 & Toluene, water, unfiltered, recoverable, micrograms per liter & E0.03 & $6300 / 5000^{c}$ \\
\hline \multicolumn{4}{|c|}{02481000 Biloxi River at Wortham, MS, Sample Date: 9/28/05, Sample Time: 1315} \\
\hline \multicolumn{4}{|c|}{ Physical properties } \\
\hline 00061 & Discharge, instantaneous, cubic feet per second & 63 & \\
\hline 63676 & Turbidity, water, unfiltered, Nephlometric Turbidity Ratio-Units & 6 & \\
\hline 00300 & Dissolved oxygen, water, unfiltered, milligrams per liter & 6.7 & 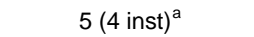 \\
\hline 00400 & $\mathrm{pH}$, water, unfiltered, field, standard units & 5.2 & $6-9^{\mathrm{a}}$ \\
\hline 00095 & Specific conductance, water, unfiltered, microsiemens per centimeter at 25 degrees Celsius & 36 & $1000^{\mathrm{a}}$ \\
\hline 00010 & Temperature, water, degrees Celsius & 26.3 & $32.2^{\mathrm{a}}$ \\
\hline \multicolumn{4}{|c|}{ Nitrogen, Phosphorus, Carbon, and Oxygen Demand } \\
\hline 00623 & Ammonia plus organic nitrogen, water, filtered, milligrams per liter as nitrogen & 0.51 & \\
\hline 00625 & Ammonia plus organic nitrogen, water, unfiltered, milligrams per liter as nitrogen & 0.53 & \\
\hline 00608 & Ammonia, water, filtered, milligrams per liter as nitrogen & E0.02 & $1.5-2^{b}$ \\
\hline 00666 & Phosphorus, water, filtered, milligrams per liter & 0.005 & \\
\hline 00340 & Chemical oxygen demand, high level, water, unfiltered, milligrams per liter & 30 & \\
\hline \multicolumn{4}{|c|}{ Major ions, metals, and related chemistry } \\
\hline 00915 & Calcium, water, filtered, milligrams per liter & 1.46 & \\
\hline 00925 & Magnesium, water, filtered, milligrams per liter & 0.834 & \\
\hline 00935 & Potassium, water, filtered, milligrams per liter & 1.23 & \\
\hline 00930 & Sodium, water, filtered, milligrams per liter & 3.09 & \\
\hline 39086 & Alkalinity, water, filtered, incremental titration, field, milligrams per liter as calcium carbonate & 2 & \\
\hline 00453 & Bicarbonate, water, filtered, incremental titration, field, milligrams per liter & 3 & \\
\hline 00940 & Chloride, water, filtered, milligrams per liter & 5.23 & $860(\mathrm{CMC}) / 230(\mathrm{CCC})^{d}$ \\
\hline 00955 & Silica, water, filtered, milligrams per liter & 14.4 & \\
\hline 00945 & Sulfate, water, filtered, milligrams per liter & 1.6 & \\
\hline 70300 & Residue on evaporation, dried at 180 degrees Celsius, water, filtered, milligrams per liter & 62 & $1,500^{\mathrm{a}}$ \\
\hline 01106 & Aluminum, water, filtered, micrograms per liter & 120 & $750(\mathrm{CMC}) / 87(\mathrm{CCC})^{\mathrm{d}}$ \\
\hline 01000 & Arsenic, water, filtered, micrograms per liter & 1 & $340 / 150^{\mathrm{a}}$ \\
\hline 01005 & Barium, water, filtered, micrograms per liter & 46 & $50,000(\text { recom })^{c}$ \\
\hline 01010 & Beryllium, water, filtered, micrograms per liter & 0.11 & $130 / 5.3^{c}$ \\
\hline 01020 & Boron, water, filtered, micrograms per liter & 19 & \\
\hline 01030 & Chromium, water, filtered, micrograms per liter & 0.41 & $16 / 11^{\mathrm{a}}$ \\
\hline 01035 & Cobalt, water, filtered, micrograms per liter & 0.652 & \\
\hline 01040 & Copper, water, filtered, micrograms per liter & 2.1 & $7 / 5^{a}$ \\
\hline 01046 & Iron, water, filtered, micrograms per liter & 400 & $1000^{d}$ \\
\hline 01049 & Lead, water, filtered, micrograms per liter & 0.57 & $30 / 1.18^{\mathrm{a}}$ \\
\hline 01130 & Lithium, water, filtered, micrograms per liter & 1.1 & \\
\hline 01056 & Manganese, water, filtered, micrograms per liter & 42 & \\
\hline 01065 & Nickel, water, filtered, micrograms per liter & 1.6 & $260 / 29^{a}$ \\
\hline 01080 & Strontium, water, filtered, micrograms per liter & 19.8 & \\
\hline 22703 & Uranium (natural), water, filtered, micrograms per liter & 0.05 & \\
\hline 01085 & Vanadium, water, filtered, micrograms per liter & 0.5 & \\
\hline 01090 & Zinc, water, filtered, micrograms per liter & 7.9 & $65 / 65^{\mathrm{a}}$ \\
\hline
\end{tabular}


Table 8. Continued--Constituents and compounds detected in samples collected in the aftermath of Hurricane Katrina, southeastern Mississippi September 19-30, 2005.

\begin{tabular}{|c|c|c|c|}
\hline $\begin{array}{c}\text { Parameter } \\
\text { code }\end{array}$ & Parameter name & Result & $\begin{array}{l}\text { Water-quality criteria } \\
\text { or recommendation }\end{array}$ \\
\hline \multicolumn{4}{|c|}{ Organic chemicals } \\
\hline 50305 & Caffeine, water, filtered, recoverable, micrograms per liter & 0.123 & \\
\hline 81552 & Acetone, water, unfiltered, recoverable, micrograms per liter & E2 & \\
\hline 34010 & Toluene, water, unfiltered, recoverable, micrograms per liter & E0.05 & $6300 / 5000^{c}$ \\
\hline \multicolumn{4}{|c|}{02481510 Wolf River near Landon, MS, Sample Date: 9/21/05, Sample Time: 1430} \\
\hline \multicolumn{4}{|c|}{ Physical properties } \\
\hline 00061 & Discharge, instantaneous, cubic feet per second & 198 & \\
\hline 00300 & Dissolved oxygen, water, unfiltered, milligrams per liter & 7.4 & 5 (4 inst) $)^{a}$ \\
\hline 00400 & $\mathrm{pH}$, water, unfiltered, field, standard units & 5.4 & $6-9^{a}$ \\
\hline 00095 & Specific conductance, water, unfiltered, microsiemens per centimeter at 25 degrees Celsius & 37 & $1000^{\mathrm{a}}$ \\
\hline 00010 & Temperature, water, degrees Celsius & 29.3 & $32.2^{\mathrm{a}}$ \\
\hline \multicolumn{4}{|c|}{ Nitrogen, Phosphorus, Carbon, and Oxygen Demand } \\
\hline 00623 & Ammonia plus organic nitrogen, water, filtered, milligrams per liter as nitrogen & 0.42 & \\
\hline 00625 & Ammonia plus organic nitrogen, water, unfiltered, milligrams per liter as nitrogen & 0.41 & \\
\hline 00666 & Phosphorus, water, filtered, milligrams per liter & 0.006 & \\
\hline 00665 & Phosphorus, water, unfiltered, milligrams per liter & 0.02 & $0.1^{\mathrm{c}}$ \\
\hline 00680 & Organic carbon, water, unfiltered, milligrams per liter & 9.5 & \\
\hline 00340 & Chemical oxygen demand, high level, water, unfiltered, milligrams per liter & 20 & \\
\hline \multicolumn{4}{|c|}{ Major ions, metals, and related chemistry } \\
\hline 00915 & Calcium, water, filtered, milligrams per liter & 1.79 & \\
\hline 00925 & Magnesium, water, filtered, milligrams per liter & 1.02 & \\
\hline 00935 & Potassium, water, filtered, milligrams per liter & 1.29 & \\
\hline 00930 & Sodium, water, filtered, milligrams per liter & 2.73 & \\
\hline 00940 & Chloride, water, filtered, milligrams per liter & 5 & $860(\mathrm{CMC}) / 230(\mathrm{CCC})^{d}$ \\
\hline 00955 & Silica, water, filtered, milligrams per liter & 12.8 & \\
\hline 00945 & Sulfate, water, filtered, milligrams per liter & 2.3 & \\
\hline 70300 & Residue on evaporation, dried at 180 degrees Celsius, water, filtered, milligrams per liter & 55 & $1,500^{\mathrm{a}}$ \\
\hline 01106 & Aluminum, water, filtered, micrograms per liter & 79 & $750(\mathrm{CMC}) / 87(\mathrm{CCC})^{\mathrm{d}}$ \\
\hline 01000 & Arsenic, water, filtered, micrograms per liter & 1.1 & $340 / 150^{\mathrm{a}}$ \\
\hline 01005 & Barium, water, filtered, micrograms per liter & 43 & $50,000(\text { recom })^{c}$ \\
\hline 01010 & Beryllium, water, filtered, micrograms per liter & 0.16 & $130 / 5.3^{c}$ \\
\hline 01020 & Boron, water, filtered, micrograms per liter & 12 & \\
\hline 01025 & Cadmium, water, filtered, micrograms per liter & E0.02 & $1.74 / 0.62^{\mathrm{a}}$ \\
\hline 01030 & Chromium, water, filtered, micrograms per liter & 0.33 & $16 / 11^{\mathrm{a}}$ \\
\hline 01035 & Cobalt, water, filtered, micrograms per liter & 0.864 & \\
\hline 01040 & Copper, water, filtered, micrograms per liter & 0.9 & $7 / 5^{\mathrm{a}}$ \\
\hline 01046 & Iron, water, filtered, micrograms per liter & 327 & $1000^{d}$ \\
\hline 01049 & Lead, water, filtered, micrograms per liter & 0.18 & $30 / 1.18^{\mathrm{a}}$ \\
\hline 01130 & Lithium, water, filtered, micrograms per liter & 1.5 & \\
\hline 01056 & Manganese, water, filtered, micrograms per liter & 68.6 & \\
\hline 01065 & Nickel, water, filtered, micrograms per liter & 1.5 & $260 / 29^{a}$ \\
\hline 01145 & Selenium, water, filtered, micrograms per liter & E0.4 & $11.8 / 4.6^{\mathrm{a}}$ \\
\hline 01080 & Strontium, water, filtered, micrograms per liter & 29.2 & \\
\hline 22703 & Uranium (natural), water, filtered, micrograms per liter & 0.05 & \\
\hline 01085 & Vanadium, water, filtered, micrograms per liter & 0.4 & \\
\hline 01090 & Zinc, water, filtered, micrograms per liter & 2.2 & $65 / 65^{\mathrm{a}}$ \\
\hline \multicolumn{4}{|c|}{ Organic chemicals } \\
\hline 50305 & Caffeine, water, filtered, recoverable, micrograms per liter & E0.164 & \\
\hline 81552 & Acetone, water, unfiltered, recoverable, micrograms per liter & E3 & \\
\hline 34010 & Toluene, water, unfiltered, recoverable, micrograms per liter & 0.13 & $6300 / 5000^{c}$ \\
\hline \multicolumn{4}{|c|}{02481510 Wolf River near Landon, MS, Sample Date: 9/28/05, Sample Time: 1615} \\
\hline \multicolumn{4}{|c|}{ Physical properties } \\
\hline 00061 & Discharge, instantaneous, cubic feet per second & 231 & \\
\hline 63676 & Turbidity, water, unfiltered, Nephlometric Turbidity Ratio-Units & 7 & \\
\hline 00300 & Dissolved oxygen, water, unfiltered, milligrams per liter & 7 & $5(4 \text { inst })^{a}$ \\
\hline 00400 & $\mathrm{pH}$, water, unfiltered, field, standard units & 5.4 & $6-9^{a}$ \\
\hline 00095 & Specific conductance, water, unfiltered, microsiemens per centimeter at 25 degrees Celsius & 38 & $1000^{\mathrm{a}}$ \\
\hline 00010 & Temperature, water, degrees Celsius & 28.2 & $32.2^{\mathrm{a}}$ \\
\hline
\end{tabular}


Table 8. Continued--Constituents and compounds detected in samples collected in the aftermath of Hurricane Katrina, southeastern Mississippi September 19-30, 2005.

\begin{tabular}{|c|c|c|c|}
\hline $\begin{array}{l}\text { Parameter } \\
\text { code }\end{array}$ & Parameter name & Result & $\begin{array}{l}\text { Water-quality criteria } \\
\text { or recommendation }\end{array}$ \\
\hline \multicolumn{4}{|c|}{ Nitrogen, Phosphorus, Carbon, and Oxygen Demand } \\
\hline 00623 & Ammonia plus organic nitrogen, water, filtered, milligrams per liter as nitrogen & 0.42 & \\
\hline 00625 & Ammonia plus organic nitrogen, water, unfiltered, milligrams per liter as nitrogen & 0.47 & \\
\hline 00666 & Phosphorus, water, filtered, milligrams per liter & 0.005 & \\
\hline 00665 & Phosphorus, water, unfiltered, milligrams per liter & 0.021 & $0.1^{\mathrm{c}}$ \\
\hline 00680 & Organic carbon, water, unfiltered, milligrams per liter & 12.6 & \\
\hline 00340 & Chemical oxygen demand, high level, water, unfiltered, milligrams per liter & 30 & \\
\hline \multicolumn{4}{|c|}{ Major ions, metals, and related chemistry } \\
\hline 00915 & Calcium, water, filtered, milligrams per liter & 1.61 & \\
\hline 00925 & Magnesium, water, filtered, milligrams per liter & 0.932 & \\
\hline 00935 & Potassium, water, filtered, milligrams per liter & 1.63 & \\
\hline 00930 & Sodium, water, filtered, milligrams per liter & 2.82 & \\
\hline 39086 & Alkalinity, water, filtered, incremental titration, field, milligrams per liter as calcium carbonate & 3 & \\
\hline 00453 & Bicarbonate, water, filtered, incremental titration, field, milligrams per liter & 3 & \\
\hline 00955 & Silica, water, filtered, milligrams per liter & 12.4 & \\
\hline 00945 & Sulfate, water, filtered, milligrams per liter & 2 & \\
\hline 70300 & Residue on evaporation, dried at 180 degrees Celsius, water, filtered, milligrams per liter & 58 & $1,500^{\mathrm{a}}$ \\
\hline 01106 & Aluminum, water, filtered, micrograms per liter & 97 & $750(\mathrm{CMC}) / 87(\mathrm{CCC})^{\mathrm{d}}$ \\
\hline 01000 & Arsenic, water, filtered, micrograms per liter & 0.9 & $340 / 150^{a}$ \\
\hline 01005 & Barium, water, filtered, micrograms per liter & 43 & $50,000(\text { recom })^{c}$ \\
\hline 01010 & Beryllium, water, filtered, micrograms per liter & 0.1 & $130 / 5.3^{\mathrm{c}}$ \\
\hline 01020 & Boron, water, filtered, micrograms per liter & 16 & \\
\hline 01030 & Chromium, water, filtered, micrograms per liter & 0.34 & $16 / 11^{\mathrm{a}}$ \\
\hline 01035 & Cobalt, water, filtered, micrograms per liter & 0.878 & \\
\hline 01040 & Copper, water, filtered, micrograms per liter & 1.9 & $7 / 5^{\mathrm{a}}$ \\
\hline 01046 & Iron, water, filtered, micrograms per liter & 519 & $1000^{d}$ \\
\hline 01049 & Lead, water, filtered, micrograms per liter & 0.2 & $30 / 1.18^{a}$ \\
\hline 01130 & Lithium, water, filtered, micrograms per liter & 1 & \\
\hline 01056 & Manganese, water, filtered, micrograms per liter & 66.3 & \\
\hline 01065 & Nickel, water, filtered, micrograms per liter & 1.58 & $260 / 29^{a}$ \\
\hline 01085 & Vanadium, water, filtered, micrograms per liter & 0.34 & \\
\hline 01090 & Zinc, water, filtered, micrograms per liter & 3.5 & $65 / 65^{a}$ \\
\hline \multicolumn{4}{|c|}{ Organic chemicals } \\
\hline 50305 & Caffeine, water, filtered, recoverable, micrograms per liter & 0.154 & \\
\hline 81552 & Acetone, water, unfiltered, recoverable, micrograms per liter & E3 & \\
\hline 34010 & Toluene, water, unfiltered, recoverable, micrograms per liter & E0.06 & $6300 / 5000^{c}$ \\
\hline \multicolumn{4}{|c|}{02481660 Jourdan River near Bay St. Louis, MS, Sample Date: 9/21/05, Sample Time: 1715} \\
\hline \multicolumn{4}{|c|}{ Physical properties } \\
\hline 00061 & Discharge, instantaneous, cubic feet per second & 3540 & \\
\hline 00300 & Dissolved oxygen, water, unfiltered, milligrams per liter & 5.9 & 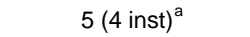 \\
\hline 00400 & $\mathrm{pH}$, water, unfiltered, field, standard units & 5.8 & $6-9^{a}$ \\
\hline 00095 & Specific conductance, water, unfiltered, microsiemens per centimeter at 25 degrees Celsius & 102 & $1000^{\mathrm{a}}$ \\
\hline 00010 & Temperature, water, degrees Celsius & 29.7 & $32.2^{\mathrm{a}}$ \\
\hline \multicolumn{4}{|c|}{ Nitrogen, Phosphorus, Carbon, and Oxygen Demand } \\
\hline 00623 & Ammonia plus organic nitrogen, water, filtered, milligrams per liter as nitrogen & 0.33 & \\
\hline 00625 & Ammonia plus organic nitrogen, water, unfiltered, milligrams per liter as nitrogen & 0.42 & \\
\hline 00666 & Phosphorus, water, filtered, milligrams per liter & 0.006 & \\
\hline 00665 & Phosphorus, water, unfiltered, milligrams per liter & 0.04 & $0.1^{\mathrm{c}}$ \\
\hline 00680 & Organic carbon, water, unfiltered, milligrams per liter & 10.5 & \\
\hline 00340 & Chemical oxygen demand, high level, water, unfiltered, milligrams per liter & 20 & \\
\hline \multicolumn{4}{|c|}{ Major ions, metals, and related chemistry } \\
\hline 00915 & Calcium, water, filtered, milligrams per liter & 1.81 & \\
\hline 00925 & Magnesium, water, filtered, milligrams per liter & 1.81 & \\
\hline 00935 & Potassium, water, filtered, milligrams per liter & 1.41 & \\
\hline 00930 & Sodium, water, filtered, milligrams per liter & 12.2 & \\
\hline 00940 & Chloride, water, filtered, milligrams per liter & 20.3 & $860($ CMC $) / 230(C C C)^{d}$ \\
\hline 00955 & Silica, water, filtered, milligrams per liter & 10.9 & \\
\hline 00945 & Sulfate, water, filtered, milligrams per liter & 3 & \\
\hline 70300 & Residue on evaporation, dried at 180 degrees Celsius, water, filtered, milligrams per liter & 80 & $1,500^{\mathrm{a}}$ \\
\hline 01106 & Aluminum, water, filtered, micrograms per liter & 58 & $750(\mathrm{CMC}) / 87(\mathrm{CCC})^{\mathrm{d}}$ \\
\hline 01000 & Arsenic, water, filtered, micrograms per liter & 0.9 & $340 / 150^{\mathrm{a}}$ \\
\hline
\end{tabular}


Table 8. Continued--Constituents and compounds detected in samples collected in the aftermath of Hurricane Katrina, southeastern Mississippi September 19-30, 2005.

\begin{tabular}{|c|c|c|c|}
\hline $\begin{array}{c}\text { Parameter } \\
\text { code }\end{array}$ & Parameter name & Result & $\begin{array}{l}\text { Water-quality criteria } \\
\text { or recommendation }\end{array}$ \\
\hline 01005 & Barium, water, filtered, micrograms per liter & 34 & $50,000(\text { recom })^{\mathrm{c}}$ \\
\hline 01010 & Beryllium, water, filtered, micrograms per liter & 0.07 & $130 / 5.3^{c}$ \\
\hline 01020 & Boron, water, filtered, micrograms per liter & 21 & \\
\hline 01025 & Cadmium, water, filtered, micrograms per liter & 0.04 & $1.74 / 0.62^{\mathrm{a}}$ \\
\hline 01030 & Chromium, water, filtered, micrograms per liter & 0.35 & $16 / 11^{\mathrm{a}}$ \\
\hline 01035 & Cobalt, water, filtered, micrograms per liter & 0.442 & \\
\hline 01040 & Copper, water, filtered, micrograms per liter & 5 & $7 / 5^{\mathrm{a}}$ \\
\hline 01046 & Iron, water, filtered, micrograms per liter & 210 & $1000^{d}$ \\
\hline 01049 & Lead, water, filtered, micrograms per liter & 0.18 & $30 / 1.18^{\mathrm{a}}$ \\
\hline 01130 & Lithium, water, filtered, micrograms per liter & 1.4 & \\
\hline 01056 & Manganese, water, filtered, micrograms per liter & 76.4 & \\
\hline 01065 & Nickel, water, filtered, micrograms per liter & 0.88 & $260 / 29^{\mathrm{a}}$ \\
\hline 01145 & Selenium, water, filtered, micrograms per liter & E0.2 & $11.8 / 4.6^{\mathrm{a}}$ \\
\hline 01080 & Strontium, water, filtered, micrograms per liter & 23.9 & \\
\hline 01085 & Vanadium, water, filtered, micrograms per liter & 0.2 & \\
\hline 01090 & Zinc, water, filtered, micrograms per liter & 4.6 & $65 / 65^{\mathrm{a}}$ \\
\hline \multicolumn{4}{|c|}{ Organic chemicals } \\
\hline 39632 & Atrazine, water, filtered, recoverable, micrograms per liter & E0.006 & $1,500^{\mathrm{e}}$ \\
\hline 50305 & Caffeine, water, filtered, recoverable, micrograms per liter & E0.117 & \\
\hline 62082 & DEET, water, filtered, recoverable, micrograms per liter & E0.1 & \\
\hline 39572 & Diazinon, water, filtered, recoverable, micrograms per liter & 0.065 & $0.1^{f}$ \\
\hline 62167 & Fipronil sulfide, water, filtered, recoverable, micrograms per liter & E0.007 & \\
\hline 38775 & Dichlorvos, water, filtered, recoverable, micrograms per liter & E0.17 & \\
\hline \multicolumn{4}{|c|}{02481660 Jourdan River near Bay St. Louis, MS, Sample Date: 9/28/05, Sample Time: 1830} \\
\hline \multicolumn{4}{|c|}{ Physical properties } \\
\hline 00061 & Discharge, instantaneous, cubic feet per second & 3400 & \\
\hline 63676 & Turbidity, water, unfiltered, Nephlometric Turbidity Ratio-Units & 6 & \\
\hline 00300 & Dissolved oxygen, water, unfiltered, milligrams per liter & 2.3 & 5 (4 inst) $^{\mathrm{a}}$ \\
\hline 00400 & $\mathrm{pH}$, water, unfiltered, field, standard units & 5.3 & $6-9^{a}$ \\
\hline 00095 & Specific conductance, water, unfiltered, microsiemens per centimeter at 25 degrees Celsius & 188 & $1000^{\mathrm{a}}$ \\
\hline 00623 & Ammonia plus organic nitrogen, water, filtered, milligrams per liter as nitrogen & 0.46 & \\
\hline 00625 & Ammonia plus organic nitrogen, water, unfiltered, milligrams per liter as nitrogen & 0.57 & \\
\hline 00608 & Ammonia, water, filtered, milligrams per liter as nitrogen & E0.02 & $1.5-2^{b}$ \\
\hline 00666 & Phosphorus, water, filtered, milligrams per liter & 0.005 & \\
\hline 00665 & Phosphorus, water, unfiltered, milligrams per liter & 0.036 & $0.1^{\mathrm{c}}$ \\
\hline 00680 & Organic carbon, water, unfiltered, milligrams per liter & 15.3 & \\
\hline 00310 & Biochemical oxygen demand, water, unfiltered, 5 days at 20 degrees Celsius, milligrams per liter & 2 & \\
\hline 00340 & Chemical oxygen demand, high level, water, unfiltered, milligrams per liter & 30 & \\
\hline \multicolumn{4}{|c|}{ Major ions, metals, and related chemistry } \\
\hline 00915 & Calcium, water, filtered, milligrams per liter & 2.48 & \\
\hline 00925 & Magnesium, water, filtered, milligrams per liter & 2.98 & \\
\hline 00935 & Potassium, water, filtered, milligrams per liter & 2.81 & \\
\hline 00930 & Sodium, water, filtered, milligrams per liter & 23.2 & \\
\hline 39086 & Alkalinity, water, filtered, incremental titration, field, milligrams per liter as calcium carbonate & 4 & \\
\hline 00453 & Bicarbonate, water, filtered, incremental titration, field, milligrams per liter & 5 & \\
\hline 00940 & Chloride, water, filtered, milligrams per liter & 41.3 & $860(\mathrm{CMC}) / 230(\mathrm{CCC})^{d}$ \\
\hline 00955 & Silica, water, filtered, milligrams per liter & 11.3 & \\
\hline 00945 & Sulfate, water, filtered, milligrams per liter & 5.2 & \\
\hline 70300 & Residue on evaporation, dried at 180 degrees Celsius, water, filtered, milligrams per liter & 136 & $1,500^{\mathrm{a}}$ \\
\hline 01106 & Aluminum, water, filtered, micrograms per liter & 107 & $750(\mathrm{CMC}) / 87(\mathrm{CCC})^{\mathrm{d}}$ \\
\hline 01000 & Arsenic, water, filtered, micrograms per liter & 1 & $340 / 150^{a}$ \\
\hline 01005 & Barium, water, filtered, micrograms per liter & 35 & $50,000(\text { recom })^{c}$ \\
\hline 01010 & Beryllium, water, filtered, micrograms per liter & 0.06 & $130 / 5.3^{c}$ \\
\hline 01020 & Boron, water, filtered, micrograms per liter & 35 & \\
\hline 01025 & Cadmium, water, filtered, micrograms per liter & E0.03 & $1.74 / 0.62^{\mathrm{a}}$ \\
\hline 01030 & Chromium, water, filtered, micrograms per liter & 0.42 & $16 / 11^{\mathrm{a}}$ \\
\hline 01035 & Cobalt, water, filtered, micrograms per liter & 1.06 & \\
\hline 01040 & Copper, water, filtered, micrograms per liter & 1.2 & $7 / 5^{\mathrm{a}}$ \\
\hline 01046 & Iron, water, filtered, micrograms per liter & 293 & $1000^{d}$ \\
\hline 01049 & Lead, water, filtered, micrograms per liter & 0.23 & $30 / 1.18^{\mathrm{a}}$ \\
\hline 01130 & Lithium, water, filtered, micrograms per liter & 1.2 & \\
\hline
\end{tabular}


Table 8. Continued--Constituents and compounds detected in samples collected in the aftermath of Hurricane Katrina, southeastern Mississippi September 19-30, 2005.

\begin{tabular}{|c|c|c|c|}
\hline $\begin{array}{l}\text { Parameter } \\
\text { code }\end{array}$ & Parameter name & Result & $\begin{array}{l}\text { Water-quality criteria } \\
\text { or recommendation }\end{array}$ \\
\hline 01056 & Manganese, water, filtered, micrograms per liter & 154 & \\
\hline 01065 & Nickel, water, filtered, micrograms per liter & 1.01 & $260 / 29^{a}$ \\
\hline 01080 & Strontium, water, filtered, micrograms per liter & 29.5 & \\
\hline 01057 & Thallium, water, filtered, micrograms per liter & E0.02 & $1400 / 40^{c}$ \\
\hline 22703 & Uranium (natural), water, filtered, micrograms per liter & 0.04 & \\
\hline 01085 & Vanadium, water, filtered, micrograms per liter & 0.3 & \\
\hline \multicolumn{4}{|c|}{ Organic chemicals } \\
\hline 50305 & Caffeine, water, filtered, recoverable, micrograms per liter & 0.125 & \\
\hline 77356 & 4-Isopropyltoluene, water, unfiltered, recoverable, micrograms per liter & E0.02 & \\
\hline 34010 & Toluene, water, unfiltered, recoverable, micrograms per liter & E0.05 & $6300 / 5000^{c}$ \\
\hline
\end{tabular}

${ }^{a}$ Water quality criteria for intrastate, interstate, and coastal waters, State of Mississippi (State of Mississippi, 2003). Criterion listed here for chromium is for chromium IV, and USGS chromium data listed here are for total chromium including both chromium III and chromium IV; criterion listed here for arsenic is for arsenic III, and USGS arsenic values are for total arsenic; criteria listed here for dissolved oxygen, $\mathrm{pH}$, and temperature are minimum criteria for all waters in Mississippi; criteria listed here for specific conductance and total dissolved solids are for Mississippi waters designated as recreation.

${ }^{b}$ Aquatic life ambient water quality criteria for ammonia update (U.S. Environmental Protection Agency, 1999). Ammonia criteria listed here are based on temperature, $\mathrm{pH}$, and life-stage. This update is adopted by Mississippi as the current criteria for ammonia.

'U.S. Environmental Protection Agency "Gold Book" standards (U.S. Environmental Protection Agency, 1986). Barium criterion listed here is a recommended standard. Total phosphorus criterion listed here is recommended for flowing waters that do not discharge into a lake or reservoir.

dU.S Environmental Protection Agency National recommended water quality criteria (U.S. Environmental Protection Agency, 2004b). Criteria for chloride, aluminum, and iron are considered criteria for non-priority pollutants.

${ }^{\mathrm{e}}$ Revised draft for aquatic life for atrazine (U.S. Environmental Protection Agency, 2003b).

'Draft diazinon criteria (U.S. Environmental Protection Agency, 2003c). 
Table 9. Results of quality-assurance samples collected at water-quality sites in the aftermath of Hurricane Katrina, southeastern Mississippi, September 19-30, 2005.

[MS, Mississippi; detections above laboratory reporting levels are presented for field blank, equipment blank, and replicate samples; all results are presented for spike sample; E, estimated; <, less than]

\begin{tabular}{clc}
\hline $\begin{array}{c}\text { Parameter } \\
\text { code }\end{array}$ & Parameter name & Result \\
\hline EQUIPMENT BLANK, Sample Date - 9/26/05, Sample Time - 1500 & \\
Nitrogen, Phosphorus, Carbon, and Oxygen Demand & E0.02 \\
00608 & Ammonia, water, filtered, milligrams per liter as nitrogen & 0.16 \\
00623 & Ammonia plus organic nitrogen, water, filtered, milligrams per liter as nitrogen & E0.01 \\
Major ions, metals, and related chemistry & E0.04 \\
00915 & Calcium, water, filtered, milligrams per liter & E4 \\
00955 & Silica, water, filtered, milligrams per liter & E0.1 \\
01046 & Iron, water, filtered, micrograms per liter & E0.5 \\
01085 & Vanadium, water, filtered, micrograms per liter & E2 \\
01090 & Zinc, water, filtered, micrograms per liter & E0.2 \\
Organic chemicals & E3 \\
62085 & 4-Nonylphenol, water, filtered, recoverable, micrograms per liter & E0.01 \\
62064 & Acetophenone, water, filtered, recoverable, micrograms per liter & E0.02 \\
62083 & Diethoxynonylphenol, water, filtered, recoverable, micrograms per liter & \\
50407 & Imazethapyr, water, filtered, recoverable, micrograms per liter & \\
34010 & Toluene, water, unfiltered, recoverable, micrograms per liter &
\end{tabular}

FIELD BLANK 02479300 Red Creek at Vestry, MS, Sample Date - 9/29/05, Sample Time - 1000

Nitrogen, Phosphorus, Carbon, and Oxygen Demand

00623 Ammonia plus organic nitrogen, water, filtered, milligrams per liter as nitrogen 0.11

00340 Chemical oxygen demand, high level, water, unfiltered, milligrams per liter 60

Major ions, metals, and related chemistry

00915 Calcium, water, filtered, milligrams per liter 0.21

00925 Magnesium, water, filtered, milligrams per liter 0.008

00930 Sodium, water, filtered, milligrams per liter 0.51

00940 Chloride, water, filtered, milligrams per liter 3.73

00955 Silica, water, filtered, milligrams per liter 2.41

01106 Aluminum, water, filtered, micrograms per liter 11

01005 Barium, water, filtered, micrograms per liter 2

01025 Cadmium, water, filtered, micrograms per liter E0.03

01030 Chromium, water, filtered, micrograms per liter $\quad$ E0.02

01040 Copper, water, filtered, micrograms per liter 3.6

01049 Lead, water, filtered, micrograms per liter 0.12

01056 Manganese, water, filtered, micrograms per liter 0.2

01065 Nickel, water, filtered, micrograms per liter 0.18

01080 Strontium, water, filtered, micrograms per liter $\quad$ E0.39

01090 Zinc, water, filtered, micrograms per liter 6.8

Organic chemicals

34466 Phenol, water, filtered, recoverable, micrograms per liter E0.3

REPLICATE: 02479155 Cypress Creek near Janice, MS, Sample Date - 9/19/05, Sample Time - 1245

Nitrogen, Phosphorus, Carbon, and Oxygen Demand

00623 Ammonia plus organic nitrogen, water, filtered, milligrams per liter as nitrogen 0.4

00625 Ammonia plus organic nitrogen, water, unfiltered, milligrams per liter as nitrogen 0.34

00631 Nitrite plus nitrate, water, filtered, milligrams per liter as nitrogen E0.05

$\begin{array}{lll}00665 & \text { Phosphorus, water, unfiltered, milligrams per liter } 0.015\end{array}$

00666 Phosphorus, water, filtered, milligrams per liter E0.004

00680 Organic carbon, water, unfiltered, milligrams per liter 10

Major ions, metals, and related chemistry

00900 Hardness, water, milligrams per liter as calcium carbonate 4

00915 Calcium, water, filtered, milligrams per liter 0.74

00925 Magnesium, water, filtered, milligrams per liter 0.496

00935 Potassium, water, filtered, milligrams per liter 0.67

00930 Sodium, water, filtered, milligrams per liter 2.27

00940 Chloride, water, filtered, milligrams per liter 3.44

00955 Silica, water, filtered, milligrams per liter 12.2 
Table 9. Continued--Results of quality-assurance samples collected at water-quality sites in the aftermath of Hurricane Katrina, southeas Mississippi, September 19-30, 2005.

\begin{tabular}{|c|c|c|}
\hline $\begin{array}{c}\text { Parameter } \\
\text { code }\end{array}$ & Parameter name & Result \\
\hline 00945 & Sulfate, water, filtered, milligrams per liter & 0.7 \\
\hline 70300 & Residue on evaporation, dried at 180 degrees Celsius, water, filtered, milligrams per liter & 38 \\
\hline 01106 & Aluminum, water, filtered, micrograms per liter & 186 \\
\hline 01000 & Arsenic, water, filtered, micrograms per liter & 0.6 \\
\hline 01005 & Barium, water, filtered, micrograms per liter & 31 \\
\hline 01010 & Beryllium, water, filtered, micrograms per liter & 0.1 \\
\hline 01020 & Boron, water, filtered, micrograms per liter & E4 \\
\hline 01030 & Chromium, water, filtered, micrograms per liter & 0.43 \\
\hline 01035 & Cobalt, water, filtered, micrograms per liter & 0.643 \\
\hline 01040 & Copper, water, filtered, micrograms per liter & 1 \\
\hline 01046 & Iron, water, filtered, micrograms per liter & 448 \\
\hline 01049 & Lead, water, filtered, micrograms per liter & 0.38 \\
\hline 01130 & Lithium, water, filtered, micrograms per liter & 0.9 \\
\hline 01056 & Manganese, water, filtered, micrograms per liter & 52.9 \\
\hline 01065 & Nickel, water, filtered, micrograms per liter & 1.09 \\
\hline 01145 & Selenium, water, filtered, micrograms per liter & E0.4 \\
\hline 01080 & Strontium, water, filtered, micrograms per liter & 8.6 \\
\hline 22703 & Uranium (natural), water, filtered, micrograms per liter & 0.06 \\
\hline 01085 & Vanadium, water, filtered, micrograms per liter & 0.5 \\
\hline 01090 & Zinc, water, filtered, micrograms per liter & 3.3 \\
\hline \multicolumn{3}{|c|}{ Organic chemicals } \\
\hline 50305 & Caffeine, water, filtered, recoverable, micrograms per liter & E0.054 \\
\hline 62070 & Camphor, water, filtered, recoverable, micrograms per liter & E0.1 \\
\hline 77356 & 4-Isopropyltoluene, water, unfiltered, recoverable, micrograms per liter & E0.02 \\
\hline 81552 & Acetone, water, unfiltered, recoverable, micrograms per liter & E3 \\
\hline 34010 & Toluene, water, unfiltered, recoverable, micrograms per liter & 0.27 \\
\hline
\end{tabular}

